

\section{Propositions}

1. Air is an important structural element for fibrous foods.

(this thesis)

2. The processing history of an ingredient is as important as its chemical composition. (this thesis)

3. Imagination is essential for generating new understanding from existing observations.

4. $\quad$ Note by Note Cuisine negates the significance of food texture.

5. Culture shock happens when making friends in a new environment, and again when returning to friends in an old environment.

6. There is no failure in gardening, only learning.

Propositions belonging to the thesis, entitled

'Multiphase effects in forming anisotropic calcium caseinate materials'

Zhaojun Wang

Wageningen, 5 July 2019 


\section{Multiphase effects in forming \\ anisotropic calcium caseinate materials}

Zhaojun Wang 


\section{Thesis committee}

\section{Promotors}

Prof. Dr Atze Jan van der Goot

Personal chair of Food Process Engineering

Wageningen University \& Research

Prof. Dr Remko M. Boom

Professor of Food Process Engineering

Wageningen University \& Research

Other members

Prof. Dr Martinus A.J.S. van Boekel, Wageningen University \& Research

Dr Peter de Jong, NIZO, Ede

Dr Wim G. Bouwman, TU Delft

Dr Julita M. van Oosten-Manski, FrieslandCampina, Wageningen

This research was conducted under the auspices of the Graduate School VLAG (Advanced Studies in Food Technology, Agrobiotechnology, Nutrition and Health Sciences). 


\title{
Multiphase effects in forming \\ anisotropic calcium caseinate materials
}

\author{
Zhaojun Wang
}

Thesis

submitted in fulfilment of the requirements for the degree of doctor

at Wageningen University

by the authority of the Rector Magnificus

Prof. Dr A.P.J. Mol

in the presence of the

Thesis Committee appointed by the Academic Board

to be defended in public

on Friday 5 July 2019

at 11 a.m. in the Aula. 
Zhaojun Wang

Multiphase effects in forming anisotropic calcium caseinate materials

130 pages

PhD thesis, Wageningen University, Wageningen, The Netherlands (2019)

With references, with summary in English

ISBN 978-94-6343-995-4

DOI $10.18174 / 477977$ 


\section{Table of contents}

$\begin{array}{ll}\text { Chapter } 1 & 1\end{array}$

General introduction

Chapter 2

9

Air bubbles in calcium caseinate fibrous material enhances anisotropy

Chapter 3

Understanding the role of air and protein phase on mechanical anisotropy of calcium caseinate fibres

Chapter 4

Thermal history of calcium caseinate affects fibre formation

Chapter 5

Maltodextrin promotes calcium caseinate fibre formation through air inclusion

Chapter 6

General discussion

References

Summary

Acknowledgement

About the author

125

List of publications

Overview of completed training activities 

Chapter 1.

General introduction 


\subsection{Protein-rich food}

Proteins are essential in foods due to their nutritional role and functional and sensory properties. Protein-rich food products are increasingly recommended for health benefits, such as weight management and prevention of muscle loss that may occur with ageing (Rondanelli et al., 2016; Shang, et al., 2018; Wang, et al., 2014). However, shortages of protein are foreseen due to the increasing world population and the increasing consumption of resource intensive food products. Unfortunately, creating sustainable and palatable protein-rich products with attractive texture imposes technological challenges. For example, high levels of protein in foods generally results in adverse effects, such as product hardening and dry mouth feel (Hogan et al., 2012). Clearly, a need exists for novel protein-rich products. Most of the protein-rich foods have developed over time via trialand -error. However, rational design of food structure based on ingredient properties and processing conditions is the future trend for the development of innovative foods.

\subsection{Anisotropy in protein-rich food}

Anisotropy is an important structural character of protein foods. This term is used to describe the directional inhomogeneity of material properties. The opposing term, isotropy, refers to physical properties that are the same in all directions (Nagashima and Mitsumata, 2011). Meat, an illustrative example of an anisotropic food product rich in protein, has a hierarchically fibrous structure, which accounts for the directional dependence of the mechanical properties of meat (Lepetit and Culioli, 1994). The anisotropy of meat is suggested to strongly influence its sensory properties, such as juiciness, chewiness and flavour release (Mioche, et al., 2003). Another example of an anisotropic food is Mozzarella cheese. It shows anisotropy due to the oriented protein fibres and elongated fat droplets as a result of stretching while heating (Bast et al., 2015; Yang et al., 2016).

It is possible to create anisotropy in other protein foods as well. Anisotropy is especially important for foods that are meant for mimicking meat. In 17 th century China, the earliest process for making meat analogues consisted of rolling thin sheets of tofu skin around a 
filling of minced and seasoned tofu skin yielding a layered anisotropic structure (Shurtleff and Aoyagi, 2016). In the modern food industry, extrusion is the most commonly used technique to create anisotropic protein foods such as meat analogues and mozzarella cheese (Lin et al., 2000; Oberg et al., 2015). A decade ago, the shear cell technology emerged, which is based on the application of shear deformation.

\subsection{Shear cell technology}

It is challenging to develop novel anisotropic food products with varying ingredients using established food structuring processes, such as extrusion. During extrusion process, food ingredients are exposed to large thermal and mechanical stresses. This has effects that are still difficult to predict and control, because of the complexity of the extrusion process. A simpler structuring process could help us gain fundamental insight in the structuring mechanisms. Thus, a shear device was designed based upon the concept of a rheometer combining simple shear deformation and large shear stresses. A picture and a schematic drawing of the device are given in Fig. 1-1. During processing, the sample filled between the cones is sheared upon rotation of the bottom cone while the top cone remains stationary. Both cones are thermostatted with a heating and a cooling water bath.

A.

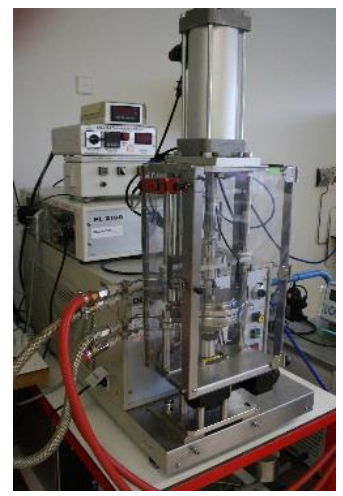

B.

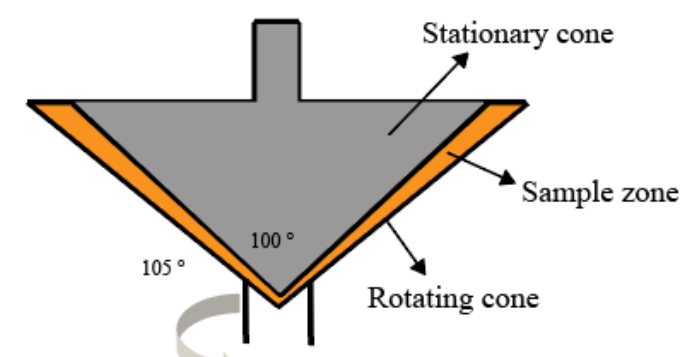

Fig. 1-1. Picture (A) and schematic picture (B) of the shear cell device.

Initially, the shear cell device was used for understanding the phenomena occurring during extrusion. Van den Einde et al. found that the degree of starch breakdown was correlated to the maximum shear stress and similar changes could be obtained by both shear cell and extrusion experiments (van den Einde et al., 2005, 2004). Later, it was 
found that shearing of wheat dough resulted in aggregation of gluten and starch patches, which could be collected into separate starch and gluten phases (Peighambardoust et al., 2008, 2006; Peressini et al., 2008). Aqueous dispersions of a dairy protein, calcium caseinate, could be transformed into hierarchically fibrous materials using shear deformation at relatively mild conditions (Manski et al., 2007a). Later, anisotropic materials with a fibrous appearance were created from plant-based ingredients, soy and gluten, when shearing at $95{ }^{\circ} \mathrm{C}$ for 15 min (Grabowska et al., 2014). In this thesis, structuring calcium caseinate will be studied in greater detail.

\subsection{Dairy proteins}

Milk contains two classes of protein: whey (20\% of the total protein content) and casein (80\%), which are either globular (whey) or random coil (casein) in nature. For consumers, the use of dairy proteins in foods is associated with high-quality nutrition. In addition, dairy proteins possess excellent technological functionality, rendering them valuable as structuring agents in many food products by ensuring stable product properties and attractive food texture.

Whey protein is a mixture of proteins isolated from whey, which is a liquid by-product of the first stage of cheese production. The most prevalent protein in whey is $\beta$-lactoglobulin, which comprises $10 \%$ of the total milk protein content. Casein consists of four different types of proteins ( $\alpha_{\mathrm{S} 1^{-}}, \alpha_{\mathrm{S} 2-}, \beta-$, and $\kappa$-casein). These caseins exhibit a strong tendency to self-assemble into casein micelles with a diameter of 100-200 nm that contain colloidal calcium phosphate (CCP) nanoclusters (De Kruif et al., 2012; De Kruif and Holt, 2003). $\alpha_{\text {s- }}$ and $\beta$-caseins are calcium-sensitive caseins that are located in nanoclusters; $\kappa$-casein, which is not susceptible to calcium, provides a sterically stabilizing layer on the micellar surface (Huppertz et al., 2017; Lucey and Horne, 2018).

Caseinates are commonly present in food products as emulsifying, foaming and texturing agents. They are extracted and purified by having the casein precipitate while the CCP is dissolved at pH $\sim 4.6$ (O’Regan and Mulvihill, 2011). After washing, the casein can be redissolved by increasing the $\mathrm{pH}$ using $\mathrm{NaOH}$ or $\mathrm{Ca}(\mathrm{OH})_{2}$. The resulting sodium and 
calcium caseinate slurries may then be spray-dried or roller-dried for further applications (Dalgleigh and Law, 1988; Fox and Mulvihill, 1982; Huppertz et al., 2017). The slurry to be dried is $\sim 20 \mathrm{wt} \%$ for spray drying and $\sim 40 \mathrm{wt} \%$ for roller drying (O'Regan and Mulvihill, 2011). Thermally induced changes are prevalent after roller drying and much less after spray drying due to the different operating temperatures (Schuck, 2011). Rollerdried caseinates are used in meat products and processed cheese because they have relatively good dispersibility and water binding capacity, while being more cost effective (Carr and Golding, 2016). The materials and process used for caseinate isolation (initial milk quality, $\mathrm{pH}$, temperature, alkali, etc.) therefore influence the properties of the final casein. Batch-to-batch differences can be observed from the same or different manufacturers. Dalgleigh and Law (1988) reported that different batches of sodium caseinate show different sensitivity to the presence of $\mathrm{Ca}^{2+}$. Bastier, et al. (1993) found that different batches of calcium caseinate had different $\mathrm{Ca}$ content and functional properties, such as water sorption capacity and viscosity, even when produced by the same manufacturer.

\subsection{Structuring of dairy proteins}

Structure formation with dairy protein has received increased attention recently. Different types of proteins (e.g. whey, $\beta$-lactoglobulin and caseinate) can be used for different food applications. For example, whey protein micro-particles are promising for improving the texture of protein foods. These particles can be prepared using heatinduced protein aggregation in combination with concurrent application of shear. A wide range of functional properties of particles can be obtained by varying process parameters (Dissanayake, et al., 2012; Peters, et al., 2015). Whey protein particles have also been considered as fat replacer in cheese (McMahon, et al., 1996; Purwanti, et al., 2013) and could to some extent replace gluten in gluten-free bread (van Riemsdijk et al., 2011). Several studies showed that whey protein particles can be used as an alternative to native protein, which could soften the texture of protein-rich foods (Purwanti et al., 2012; Sağlam et al., 2014). 
$\beta$-lactoglobulin can form fibrils (Veerman, et al., 2002; Akkermans, et al., 2008; Akkermans, et al., 2008). These fibrils are anisotropic protein aggregates with a length of 1-10 $\mu \mathrm{m}$ and a thickness of a few nanometres. These can typically be formed by protein aggregation at low $\mathrm{pH}$ and at low ionic strength. Applying concurrent shear flow enhances the fibril formation. Fibrils with a similar morphology can also be obtained with whey protein isolate (Akkermans et al., 2008; Bolder et al., 2006). Suspensions of the fibrils show rich rheological behaviour (e.g. shear thickening and shear thinning), and might also have potential to modify the texture of food products.

Concentrated calcium caseinate dispersions can be transformed into hierarchically structured, fibrous materials using shear deformation and concurrent crosslinking by transglutaminase (Fig. 1-2). The formation of such fibrous morphology was attributed to the attraction between caseinate micelles due to the presence of the divalent $\mathrm{Ca}^{2+}(\mathrm{Manski}$ et al., 2007c), resulting in mild aggregation of these aggregates. These micelles are susceptible to the shear flow and deform into long alignment of aggregates. By simultaneously solidifying with transglutaminase, these alignments can be preserved. The presence of calcium is a prerequisite, as it was found that sodium caseinate dispersion gave a isotropic structure after identical processing. This was confirmed by the observation that the formation of fibres is suppressed by the addition of a calcium chelator, being sodium triphosphate (Grabowska et al., 2012).

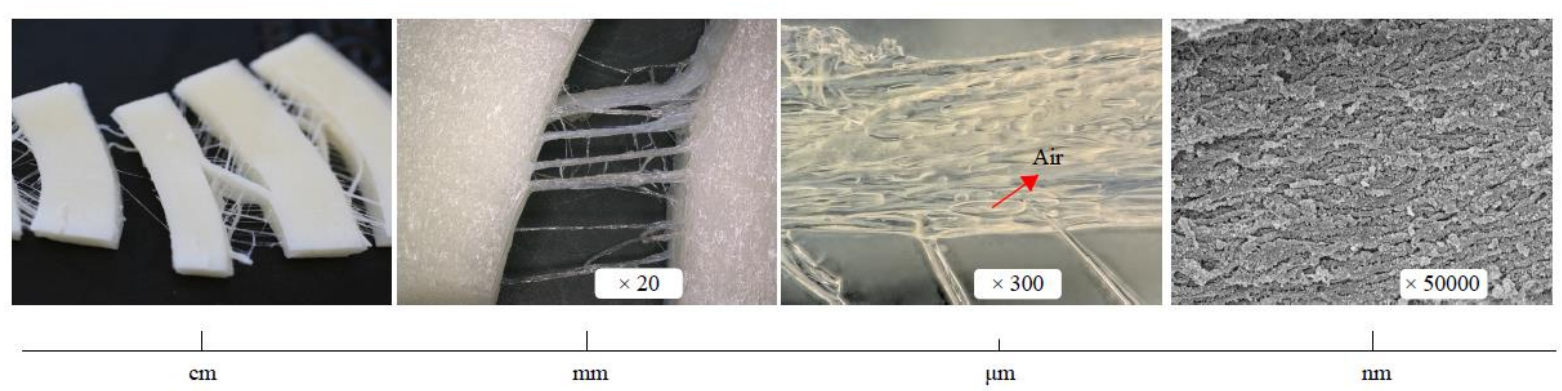

Fig. 1-2. Hierarchically fibrous structure of calcium caseinate material which was sheared at $50 \mathrm{rpm}$ for $5 \mathrm{~min}$ at $50{ }^{\circ} \mathrm{C}$ and concurrent cross-linked by transglutaminase (enzymeprotein ratio $1: 20$ ). 
The proposed mechanism was supported by the rheological characterisation of these dispersions, which could be a tool to predict the structuring potential of caseinates (Manski et al., 2007c). In a specific range of shear rates, the viscosity showed a large peak, and was strongly reduced at larger shear rates. This peak is an indication of changes in structure due to the shear deformation. Although this behaviour can qualitatively explain some of the properties of the final materials, such as their anisotropic mechanical properties, they cannot explain the observed sensitivity to the exact amount of transglutaminase added. In addition, this mechanism cannot explain the large differences obtained with different types of caseinate. It is therefore expected that this mechanism does not completely explain the creation of the hierarchal structure. Therefore, in this thesis, the formation of the fibrous structure was further investigated, to quantify the effects, and to refine the understanding of the mechanisms, to account for the difference in properties of different starting materials.

\subsection{Aim and outline of the thesis}

The aim of this thesis is to further elucidate the mechanism of anisotropy formation in dense calcium caseinate dispersions, which can help us predict the structural properties by characterizing specific initial ingredient properties. It was hypothesized that the dependence on the starting ingredient was due to its thermal history. In addition, initial experiments led us to hypothesize that the incorporation of air could be important for the final mechanical properties, and therefore special attention was paid to the role of this extra phase in the system.

In Chapter 2, we investigate the importance of air as a structural element in dense calcium caseinate dispersion. Its effect on product properties is revealed, confirming our initial hypothesis on this. The morphology of entrapped air was studied with reflective light microscopy and X-ray tomography.

The relative importance of protein and air bubble on the mechanical anisotropy of calcium caseinate fibres is further investigated in Chapter 3. Here, the effect of air on the final mechanical anisotropy of these fibrous materials was described with a load-bearing 
model. The anisotropy of the protein phase was estimated using materials obtained from deaerated dispersions after shearing at different shear rates.

Commercial calcium caseinate is available in spray-dried and roller-dried powder. Chapter 4 focuses on the differences between spray-dried and roller-dried calcium caseinate powder through investigating their physical properties and structuring potential, concluding that the thermal history of roller-dried calcium caseinate has major consequences for its fibre formation potential.

In Chapter 5, a multiphase system was introduced to obtain a fibrous structure form roller-dried calcium caseinate powder. The effect of dispersed phases, being maltodextrin domains and air bubbles, on structure formation was investigated by analysing the macro-, meso- and microstructure, and relating mechanical properties to the obtained structure.

Chapter 6 reviews the results obtained in this study and provides an overview of anisotropic structure formation principles with calcium caseinate. First, the intrinsic properties of calcium caseinate and the importance of air bubbles for fibrous structure are summarised. Then analytical techniques related to studying anisotropy are discussed. The chapter ends with a discussion about the potential of transferring the insights towards structuring of plant-based protein foods. 


\section{Chapter 2.}

\section{Air bubbles in calcium caseinate fibrous materials enhances anisotropy}

This chapter has been published as Wang, Z., Tian, B., Boom, R., \& van der Goot, A. J. (2019). Air bubbles in calcium caseinate fibrous material enhances anisotropy. Food Hydrocolloids, 87, 497-505. 


\section{Abstract}

Dense calcium caseinate dispersions can be transformed into hierarchically fibrous structures by shear deformation. This transformation can be attributed to the intrinsic properties of calcium caseinate. Depending on the dispersion preparation method, a certain amount of air gets entrapped in the sheared protein matrix. Although anisotropy is obtained in the absence of entrapped air, the fibrous appearance and mechanical anisotropy of the calcium caseinate materials are more pronounced with dispersed air present. The presence of air induces the protein fibres to be arranged in microscale bundles, and the fracture strain and stress in the parallel direction are larger compared with the material without air. The effects can be understood from the alignment of the fibres in the parallel direction, providing strain energy dissipation. This study shows that creation of anisotropy is the result of interactions between multiple phases. 


\subsection{Introduction}

A decade ago, an anisotropic calcium caseinate material was created by a novel technique based on well-defined shear flow (Manski, et al., 2007a, 2007b), which is relevant for its structural resemblance to meat. Therefore, it has a potential to be a basis of new meat analogue products. The calcium caseinate dispersion was deformed by simple shear flow and concurrent crosslinking by transglutaminase. A typical fibre diameter of $\sim 100-200$ nm was observed with scanning electron microscopy (Manski et al., 2007b). The formation of this fibrous morphology was attributed to the aggregation of caseinate micelles, which have mildly attractive interactions due to the divalent calcium ions (Manski, et al., 2007c). The aggregates are susceptible to aligning in the shear flow and simultaneously solidifying with transglutaminase. The presence of calcium ions was shown to be essential; the anisotropic structure could not be formed with sodium caseinate, and the formation of the fibrous structure was blocked by the addition of sodium triphosphate, which exchanges the divalent $\mathrm{Ca}^{2+}$ bound to the protein with $\mathrm{Na}^{+}$ (Grabowska et al., 2012).

Dispersed air was observed in these calcium caseinate fibrous materials (Manski et al., 2007b; Manski, et al., 2008), and also in materials created with soy protein isolate with wheat gluten blend (Grabowska et al., 2014) and soy protein isolate with pectin blend (Dekkers et al., 2018). A more recent study revealed that such fibrous materials may contain up to $20 \%(\mathrm{v} / \mathrm{v}$ ) air with an average bubble diameter of $\sim 100-400 \mu \mathrm{m}$ (Tian et al., 2018). The dispersed air provides an additional phase that influences the texture, microstructure, and functionality (Campbell and Mougeot, 1999; Zúñiga and Aguilera, 2009). During dough mixing, it was found that air entrapment affects the dough rheology. The rate of work input during dough mixing increased due to the presence of air bubbles while the resistance of dough to failure decreased under biaxial extension (Chin et al., 2005). A study on agar gel showed that an aerated gel is stronger than a gel without air (Ross et al., 2006; Tiwari and Bhattacharya, 2011a). Many natural porous composites, which are often hierarchically organized at multiple length scales (nano-, micro- and macroscale), have remarkable strength from the alignment of their fibres, crystals, or 
other structural elements, but have great toughness at low density (Gibson, 2012; Wegst et al., 2015); examples are bamboo and wood. The voids play a significant role in fracture toughening (Habibi and Lu, 2014; Lakes, 1993; Stanzl-Tschegg et al., 2011; Wegst et al., 2015). For wood, the pores within the structure arrest cracks by the dispersion of stress over the surface of the pore after it has been compromised (Stanzl-Tschegg et al., 2011). This also occurs in synthetic cellular composite materials, such as honeycombs (Greil et al., 2002; Haghpanah et al., 2013) and foams (Andersons et al., 2015; Olurin et al., 2001).

The entrapment of air can introduce an extra separate phase into the calcium caseinate material (Manski et al., 2008), and may well lead to different mechanical properties. In this paper, we investigate the role of air bubbles on the microstructure and mechanical properties of the material structure. The morphology of entrapped air was studied with reflective light microscopy and X-ray tomography. Scanning electron microscopy was used to study the protein structure in the vicinity of air bubbles. Differences in mechanical properties were revealed with tensile analysis.

\subsection{Materials and methods}

\subsubsection{Materials}

Spray-dried calcium caseinate was kindly provided by DMV International (Veghel, the Netherlands). The calcium caseinate powder contains $89 \mathrm{wt} \%$ protein and $1.5 \mathrm{wt} \%$ calcium according to the manufacturer's specifications. The activity of microbial $\mathrm{Ca}^{2+}$ independent transglutaminase derived from Streptoverticillium moberansae (1 wt\% transglutaminase, 99 wt\% maltodextrin; Ajinomoto Co. Inc., Tokyo, Japan) was 114 units. $\mathrm{g}^{-1}$ as determined with the hydroxamate method (Yokoyama et al., 2003). This product was referred to as "enzyme" in this paper. A 20\% (w/w in demineralized water) enzyme solution was stirred at room temperature for $1 \mathrm{~h}$, and stored at $4^{\circ} \mathrm{C}$ for 7 days before use (Grabowska et al., 2012). 


\subsubsection{Protein premixes for preparative shearing}

Five protein premixes, referred to as premixes 1-5, were prepared. All dispersions contained $30 \mathrm{wt} \%$ calcium caseinate powder, and transglutaminase was added to one of the dispersions. Premix 1 was prepared by manually mixing the protein, water and transglutaminase solution (enzyme-protein ratio 1:20) with a spatula for $1 \mathrm{~min}$. The purpose of mixing was to roughly blend protein with water with minimal mixing intensity. Premix 2 was prepared similarly to premix 1, but without the addition of transglutaminase solution. Premix 3 was prepared by manually mixing the protein and water with a spatula for $1 \mathrm{~min}$, and then resting at room temperature for an additional 1 h. Premix 4 was prepared by manually mixing the protein and water with a spatula for 1 min, then heating the dispersion at $80^{\circ} \mathrm{C}$ for $5 \mathrm{~min}$ and subsequently leaving it at room temperature for 55 min. Premix 5 was prepared similarly to premix 4 but an additional centrifugation step $(2500 \times g$ for $2 \mathrm{~min})$ was used to remove most of the air from the mixture.

\subsubsection{Sample preparation in the shear cell device}

A shear device with gap angle $2.5^{\circ}$ (Wageningen University, the Netherlands) was used to structure the dense calcium caseinate dispersions into anisotropic materials. The shear device is depicted and described in more detail by van der Zalm, Berghout, van der Goot, and Boom, (2012). The rotating cone and the stationary cone were thermostatted with a heating and a cooling water bath. The premixes were transferred to the pre-heated $\left(50^{\circ} \mathrm{C}\right)$ shearing device. The materials were made with a rotating speed of $50 \mathrm{rpm}$ for $5 \mathrm{~min}$ at $50^{\circ} \mathrm{C}$. After processing, the materials were cooled to $4{ }^{\circ} \mathrm{C}$ in $10 \mathrm{~min}$ before removing them from the device. Tensile tests were performed on materials within $1 \mathrm{~h}$ after discharge from the device. Parts of the materials were stored at $-20^{\circ} \mathrm{C}$ until further analysis.

An extra experiment was done to elucidate the effect of air incorporation in the system and to exclude that the pre-treatment influenced the structuring properties of the calcium caseinate. Premix 5 was whipped with a kitchen mixer (MUM54230/02, Bosch, Germany) at speed 6 (out of 7) for 5 min to again incorporate air in the premix before material 
preparation. The void fraction of the resulting material was $2.8 \pm 0.2 \%$. The resulting material showed similar mechanical properties and anisotropy as sample 4 (Appendix 2A).

\subsubsection{Tensile strength analysis}

A texture analyser (Instron Testing System, table model type 5564) was used with a load cell of $100 \mathrm{~N}$. A tensile bar was used to take samples $15.2 \mathrm{~mm}$ long and $3.18 \mathrm{~mm}$ wide. The thickness of the samples varied between 4 and $6 \mathrm{~mm}$. The samples were taken parallel and perpendicular to the shear flow (based on the shear-vorticity plane). The tensile tests were conducted at a constant deformation speed of $3 \mathrm{~mm} \cdot \mathrm{s}^{-1}$. Grips with abrasive paper were used to prevent the sample slipping in the grips during testing. At least three specimens per direction were measured. The measurements were recorded using a camera in a smartphone. The results obtained from these tensile tests were depicted as force-displacement curves. The true stress $(\sigma, \mathrm{kPa})$ and fracture strain $(\varepsilon,-)$ were calculated using the following equations:

$$
\begin{gathered}
\sigma=F(t) / A(t) \\
\varepsilon=\ln \left(L(t) / L_{0}\right)
\end{gathered}
$$

Where $F(t)$ was force, $A(t)$ was cross sectional area, and $L(t)$ was the length of the sample all at time $t$ and $L_{0}$ was the initial length of the tensile bar (15.2 mm). A point following a dramatically decrease of stress in the stress-strain curve was taken as fracture point. This point was used to read fracture stress $(\sigma, \mathrm{kPa})$ and fracture (Hencky) strain $(\varepsilon$, -), respectively. In this study, the apparent strain hardening coefficient $n$ was calculated by applying a power law fit $\left(\sigma=k \cdot \varepsilon^{\mathrm{n}}\right.$ ) using the Hencky strain interval between $30 \%$ and $100 \%$ of fracture strain $\varepsilon$. When $n$ exceeds 1 , the sample exhibits strain hardening. $k$ is a coefficient that correlates the stress and the stain of the materials and is therefore a measure of material stiffness. The ratio of the average data for mechanical properties measured in the parallel and perpendicular directions to the shear flow was calculated and used as an indication of the mechanical anisotropy of the samples. 


\subsubsection{X-ray tomography}

Samples were scanned using a GE Phoenix v|tome|x m tomographer (General Electric, Wunstorf, Germany) set at $80 \mathrm{kV} / 90 \mu \mathrm{A}$. The system contains two X-ray sources; the 240 $\mathrm{kV}$ micro-focus tube with a tungsten target was used. The images were recorded by a GE DXR detector array with $2024 \times 2024$ pixels (pixel size, $200 \mu \mathrm{m}$ ). The detector and object were located $815 \mathrm{~mm}$ and $28.55 \mathrm{~mm}$ from the X-ray source, respectively. This results in a spatial resolution of $7.00 \mu \mathrm{m}$. A full scan consists of 750 projections over $360^{\circ}$. Four images are taken for each projection. The first image was skipped; images 2-4 were averaged into one projection. GE reconstruction software (Wunstorf, Germany) was used to calculate the 3D structure via back projection. The 3D images were analysed using Avizo imaging software version 9.3.0, including the total sample volume, total gas volume, gas void fraction $(p)$, every single bubble volume, and its length, width and degree of deformation (Def). The degree of deformation (Def) was defined as:

$$
\operatorname{Def}=(a-b) /(a+b)
$$

where $a$ is the length of a deformed air bubble parallel to the shear flow, and $b$ is the average width of the air bubble perpendicular to the shear flow (based on velocity gradient-shear flow plane and vorticity-shear flow plane). An average value of Def was used to represent the deformation of air bubble with different bubble volume range. Def $=0$ represents a spherical air bubble; Def $=1$ represents an infinitum deformed air bubble. Three samples of each materials were measured.

\subsubsection{Scanning electron microscopy}

The sheared calcium caseinate materials were torn apart by hand along the shear flow direction (approximately $2 \mathrm{~mm}$, based on the shear flow-vorticity plane), and rinsed with demineralized water. Subsequently, the samples were fixated in a glutaraldehyde solution $(2.5 \%, v / v)$ for $1 \mathrm{~h}$ and dehydrated in a graded series of ethanol $(10 \%, 30 \%, 50 \%, 70 \%$, $90 \%$ and $100 \% \mathrm{v} / \mathrm{v}, 20 \mathrm{~min}$ each). Critical point drying with carbon dioxide (CPD 020, Balzers, Liechtenstein) was performed. The dried samples were then glued to sample 
holders using conductive carbon cement (Leit-C, Neubauer Chemicalien, Germany), and sputter coated with $15 \mathrm{~nm}$ platinum (JFC 1200, JEOL, Japan). The surface was observed with a field emission scanning electron microscope (Magellan 400, FEI, Eindhoven, the Netherlands) at ambient temperature at a working distance of 4-8 $\mathrm{mm}$, with secondary electron detection at $2 \mathrm{kV}$ and $6.3 \mathrm{pA}$.

\subsubsection{Reflective light microscopy}

The frozen materials were cut into thin slices with a razor blade (single-edge carbon steel; Electron Microscopy Sciences, Hatfiled, PA) parallel and perpendicular to the shear flow, resulting in the velocity gradient-shear flow plane and velocity gradient-vorticity plane, respectively. The samples were imaged using a digital microscope (Keyence VHX-2000E) with $100 \times$ magnification.

\subsection{Results and discussion}

\subsubsection{Macro- and Microstructure}

Fig. 2-1 shows the macrostructure of calcium caseinate materials 1-5. A highly fibrous morphology was obtained when shearing a calcium caseinate premix with transglutaminase (material 1). When this material was torn along the direction of shear flow, thin isolated fibres appeared, illustrating the pronounced fibrous character. Enzymatic crosslinking is not a prerequisite for the fibrous structure formation. Shearing $30 \mathrm{wt} \%$ calcium caseinate in the absence of transglutaminase (materials 2-5) also gives anisotropy, but the materials are stickier than material 1. Visual observation suggests that material 5 contains fewer isolated fibres than premixes 2-4. Lack of air bubbles makes the material to break in larger parts, because the material contains hardly any air bubbles along which thin fibres can appear upon tearing.

Light microscopy (Fig. 2-1) shows that materials 1-4 contained many air bubbles. The larger and well-visible air bubbles were elongated and orientated in the direction parallel to the shear flow, which reveal that the presence of the air bubbles induced structural anisotropy at microscale. Small air bubbles were still circular due to their higher Laplace 
pressure. The image of material 5 shows the isotropic microstructure without air bubbles. We speculate that the presence of air bubble and fibrousness are correlated. The main explanation could be that the materials $2-4$ break along the air bubble when tearing them along the direction of shear flow. Without air bubbles, structure will break into larger pieces upon tearing. 
Macrostructure

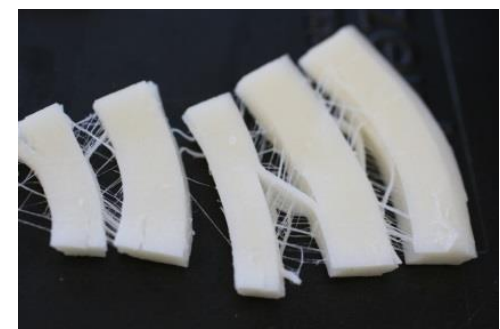

2.

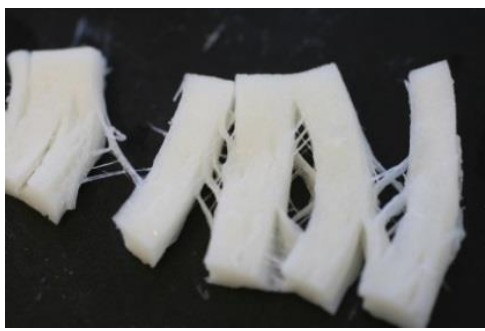

3.

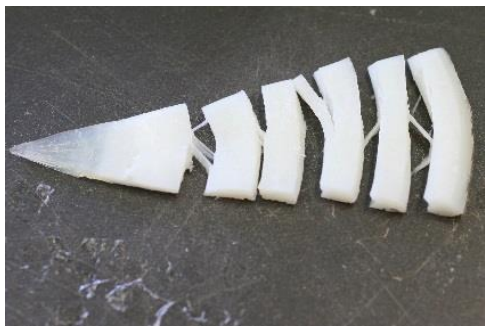

4.

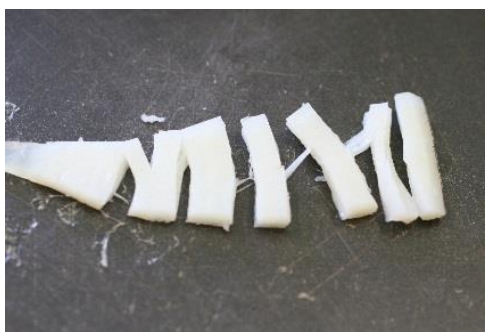

5.

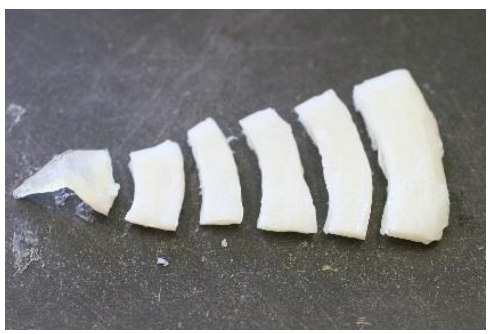

\section{Microstructure}

Parallel

Perpendicular
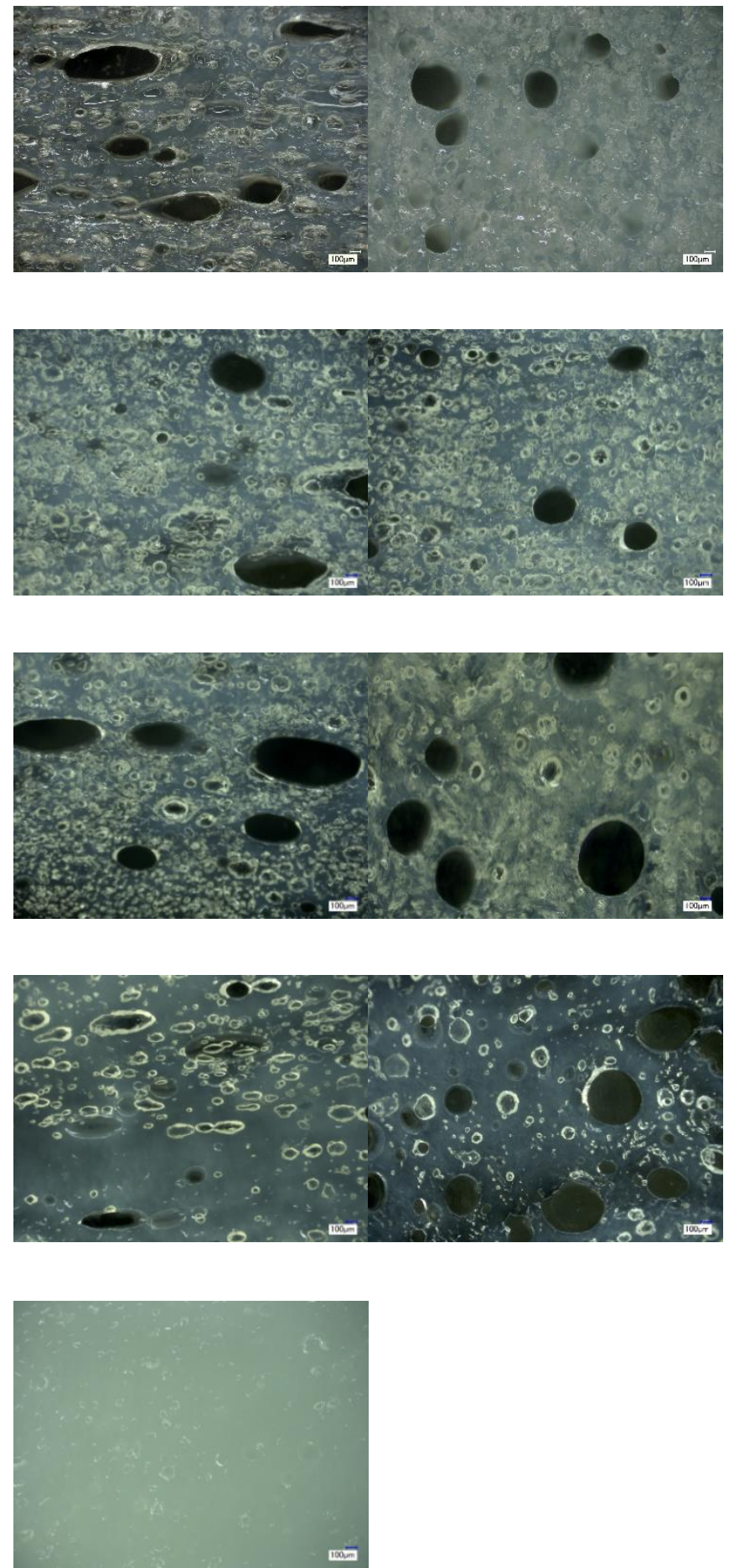

Fig. 2-1. Macrostructure and light microscopy images of $30 \mathrm{wt} \%$ calcium caseinate materials prepared with the different premixes $1-5$. The scale bars in the light microscopy images denote $100 \mu \mathrm{m}$. 


\subsubsection{The morphology of air bubbles}

2D and reconstructed 3D X-ray tomography images show the morphology of air bubbles in materials 1-4 (Fig. 2-2). These images confirmed the elongation and orientation of the air bubbles as illustrated in Fig. 2-1. It could be also deduced that the dispersed air bubbles could induce the fibres to be arranged in bundles at the microscale, thus yielding a hierarchical structure (Manski et al., 2008).
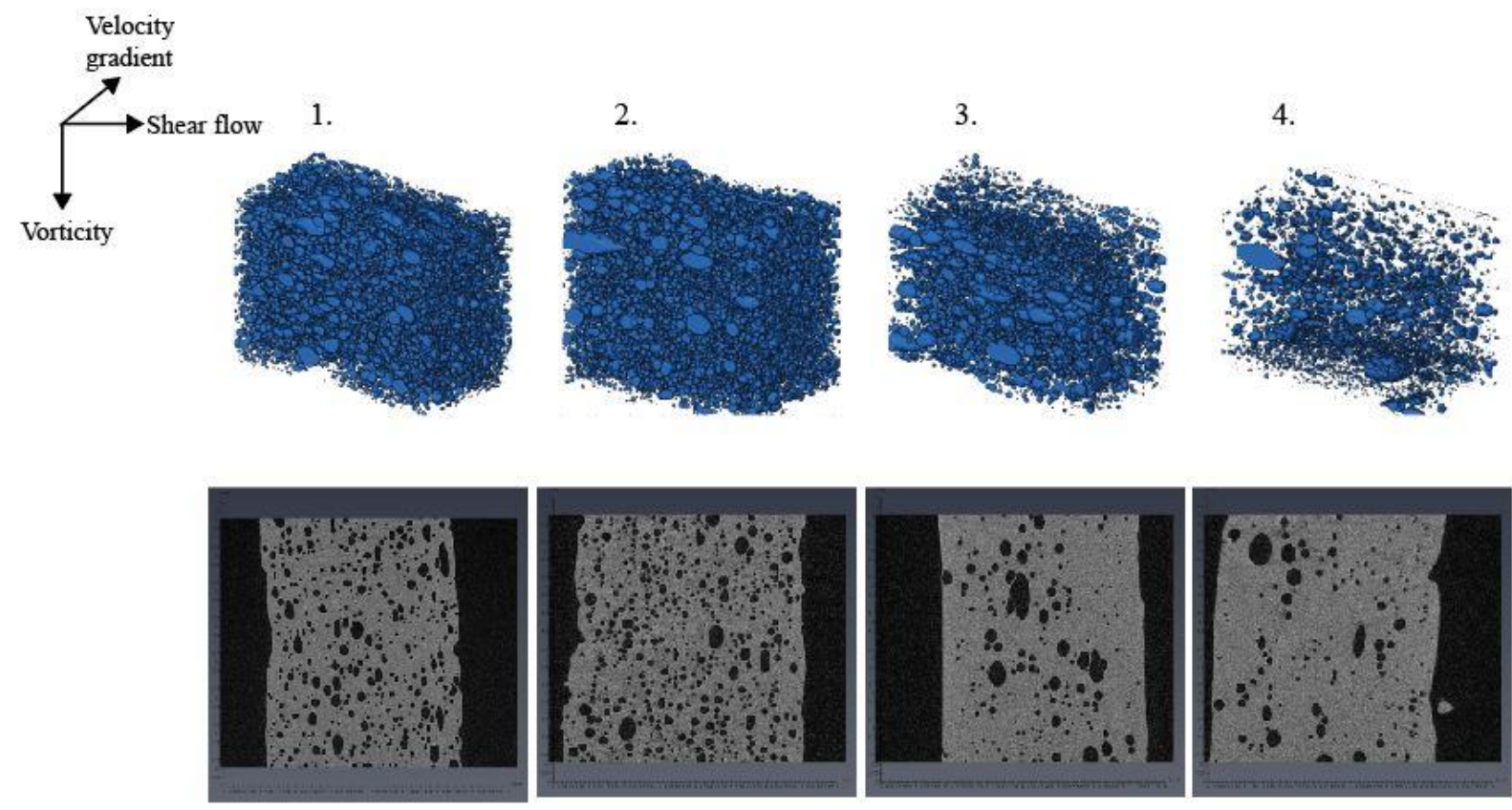

Fig. 2-2. X-ray tomography images of air bubble morphology in sheared calcium caseinate materials $1-4$.

The reconstructed 3D images were used to determine the cumulative void fractions below different bubble volumes and the associated deformation of the bubbles (Fig. 2-3). The total void fraction decreased from material 1 to 5 , mainly due to the difference in the void fraction of large air bubbles $\left(>10^{-3} \mathrm{~mm}^{3}\right)$. Material A had a higher total void fraction of air $(19.2 \pm 1.2 \%)$ than material $2(14.4 \pm 3.2 \%)$, indicating that enzymatic crosslinking resulted in more air incorporation in the protein matrix. Going from material 2 to 5 , the void fraction of air decreased further. Apparently, more pre-treatment (longer resting time and heating) of the premixes resulted in less air in the protein matrix. So far, it is not fully clear why heating and longer resting time reduced the void fractions. A possible 
explanation in case of heating is that a warmer dispersion has a lower viscosity, which enables escaping of air bubbles from the dispersion. Longer hydration also leads to a lower dispersion viscosity, because more interaction of calcium caseinate and water could lead to weakening of the dispersions. Besides, longer resting time also gives air bubbles more time to escape.
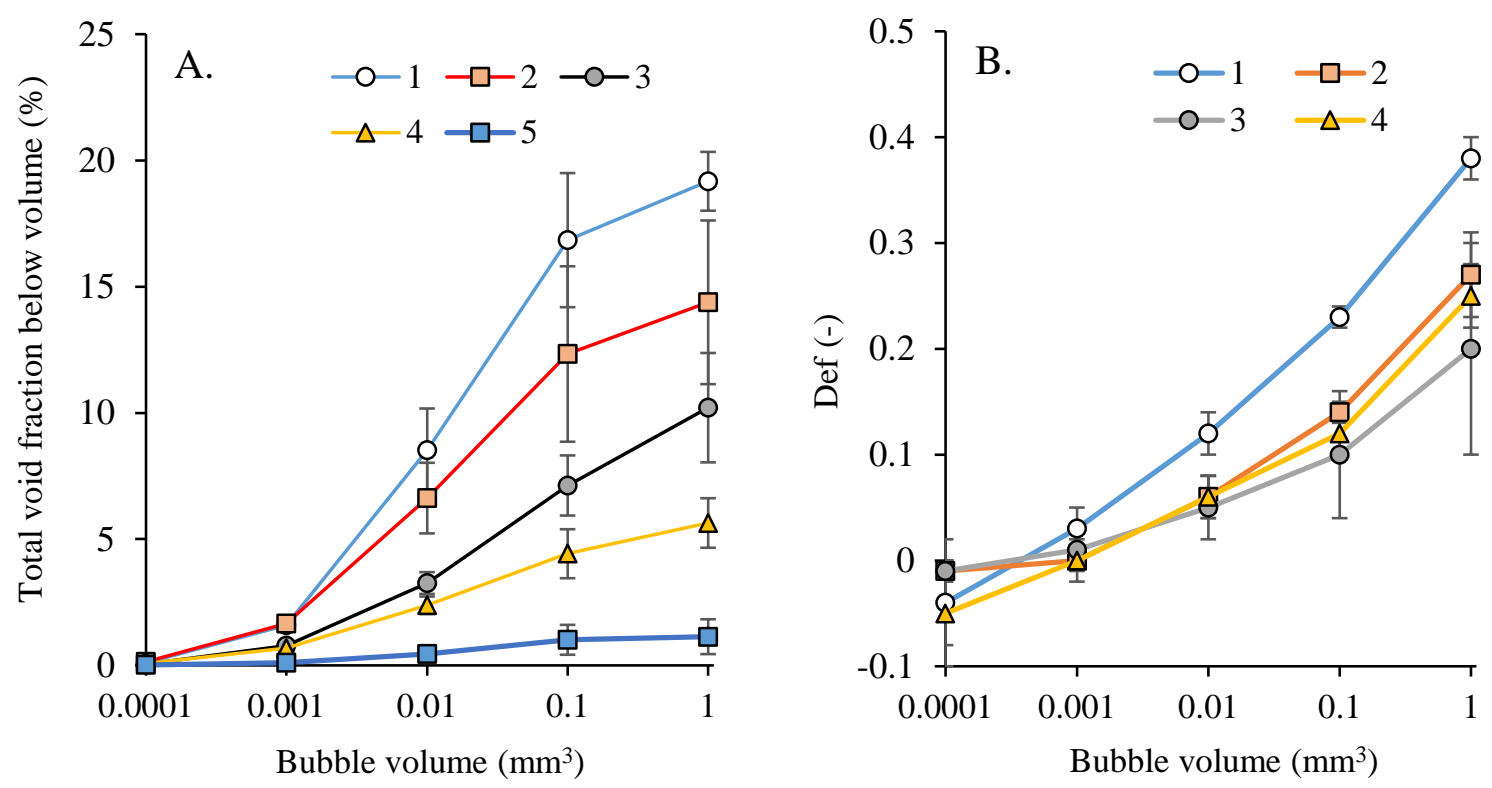

Fig. 2-3. A: Overall cumulative void fractions (as the void fraction below the bubble volume in $\left.\mathrm{mm}^{3}\right)$. B: Associated deformation of the bubbles.

The deformation of air bubbles increased with increasing bubble volume in materials 14 (Fig. 2-3B). The small air bubbles $\left(\leq 10^{-3} \mathrm{~mm}^{3}\right)$ were not deformed $(-0.05-0.03)$, whereas the large air bubbles $\left(>10^{-3} \mathrm{~mm}^{3}\right)$ were slightly deformed $(0.05-0.38)$. This is in line with the morphology of bubbles observed by light microscopy (Fig. 2-1). The deformation of the air bubbles was similar for materials $2-4$, however, material 1 showed larger deformation. This is probably due to the crosslinking in material 1 , resulting in a higher consistency during shearing and therefore larger force on the bubbles. In conclusion, the absence of transglutaminase reduced the air content and the degree of deformation. 


\subsubsection{Morphology around air bubbles}
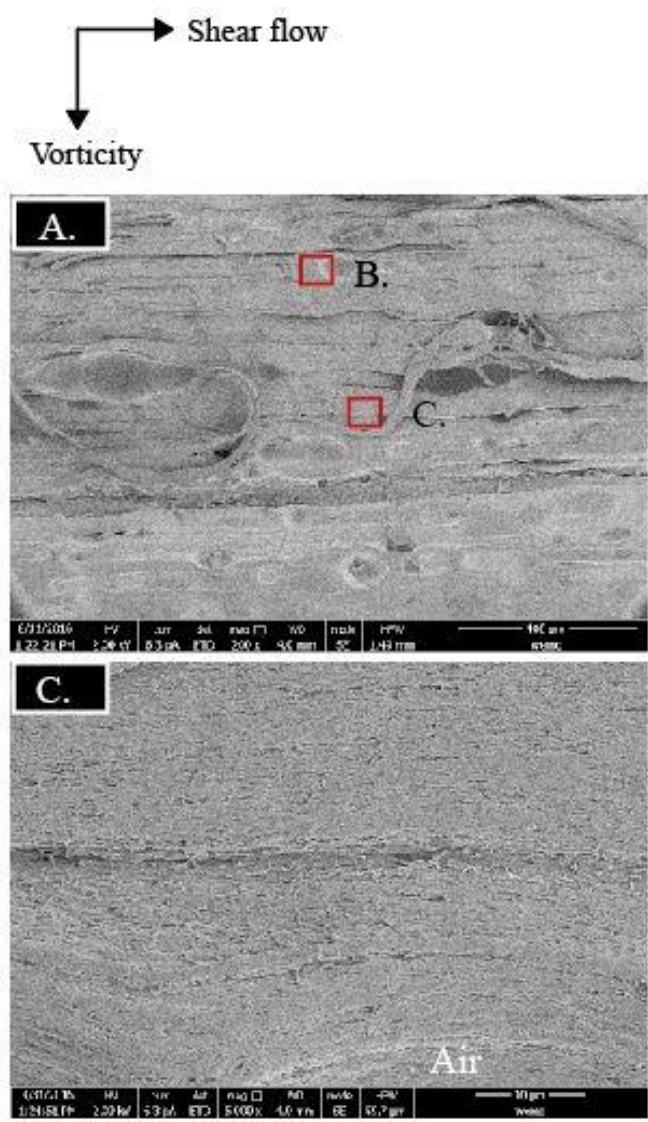

\section{E.}

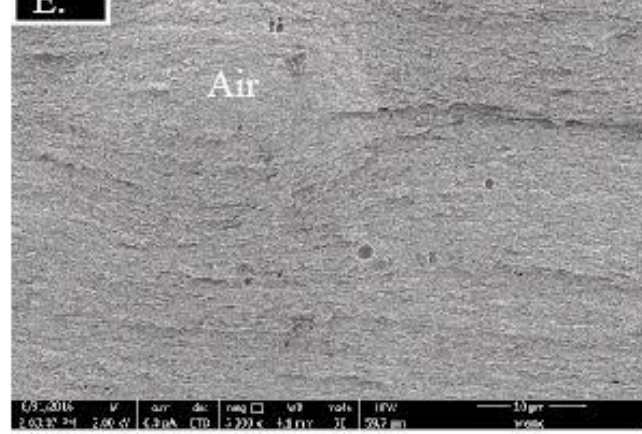

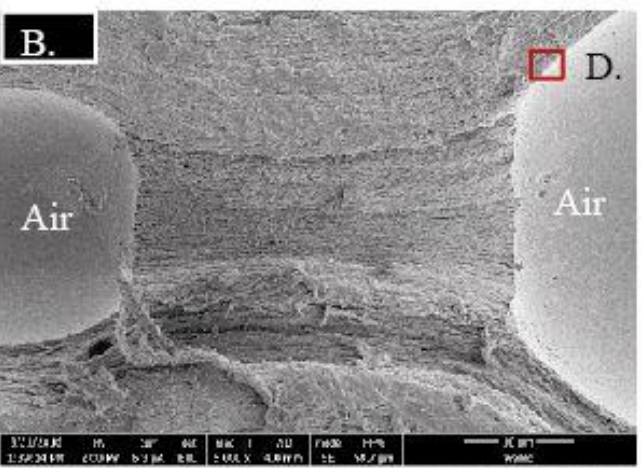

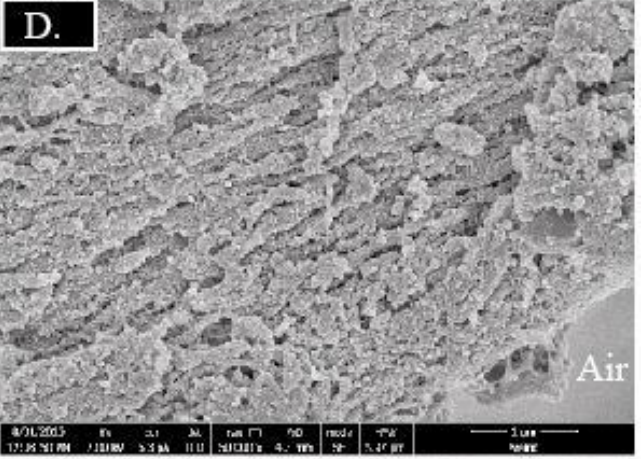

F.

Fig. 2-4. SEM images of the fractured surface parallel to the shear flow of material 1 (images A-D) and 2 (images E-F). The scale bars denote $400 \mu \mathrm{m}$ in image A, $10 \mu \mathrm{m}$ in images B, C and E, and $1 \mu \mathrm{m}$ in images D and F. Images B and C zoom in on the area outlined by the red square in image $\mathbf{A}$ at $5000 \times$. Images in $\mathbf{C}$ zoom in on the area outlined by the square in image $\mathbf{B}$ at $50,000 \times$. The air represents air bubbles in the images.

Fractured fibres on the microscale are visible parallel to the shear flow after tearing material 1 by hand along the shear flow direction (based on the shear-vorticity plane) (Fig. 2-4A). SEM images were obtained at different magnifications and locations close to 
air bubbles within this sample (Fig 2-4B, C). The fibre alignment at the bubble surface more or less follows the curvature of a bubble (Fig 2-4B, C). This is shown in more detail in Fig. 2-4D. The orientation of the fibres between two adjacent air bubbles follows the flow pattern around the bubbles (Fig. 2-4B). In the absence of transglutaminase, the fibre orientation is still present (Fig. 2-4E, F), but less distinct compared with material 1 (Fig.24B-D).

\subsubsection{Mechanical properties}

The anisotropy index is the ratio of the fracture strain or stress parallel and perpendicular to the shear flow, which was used as an indication of the mechanical anisotropy of the sheared materials. Enzymatic crosslinking results in a high anisotropy for material 1 (Fig. 2-5). The main difference in materials 2,3 , and 4 is the void fraction of air; only a slight decrease in the anisotropy was found for those materials. Thus, the different pretreatments had little effect on their mechanical anisotropy. Material 4 showed only slight anisotropy. To conclude, the mechanical anisotropy increases when transglutaminase and air bubbles are present in calcium caseinate material.

Material 1 had the largest fracture stress and strain in the parallel direction and the smallest fracture stress in the perpendicular direction; the transglutaminase-induced crosslinking increases the strength in the parallel direction but not in the perpendicular direction, which is in line with previous research (Manski, et al., 2007b). In the absence of transglutaminase, the fracture stress of materials 2-4 increased with decreasing void fraction of air in both the parallel and perpendicular directions. No significant differences were found in the fracture strain. The measurements show that material 5 , containing hardly any air, had the smallest fracture stress and strain in the direction parallel to shear flow. This indicates that the presence of air bubbles in the protein matrix reinforced the material in the parallel direction. In the perpendicular direction, all the materials become weaker with increased air content. In the perpendicular direction, air bubbles act as a weak phase, which promotes crack propagation along the fibre direction. 

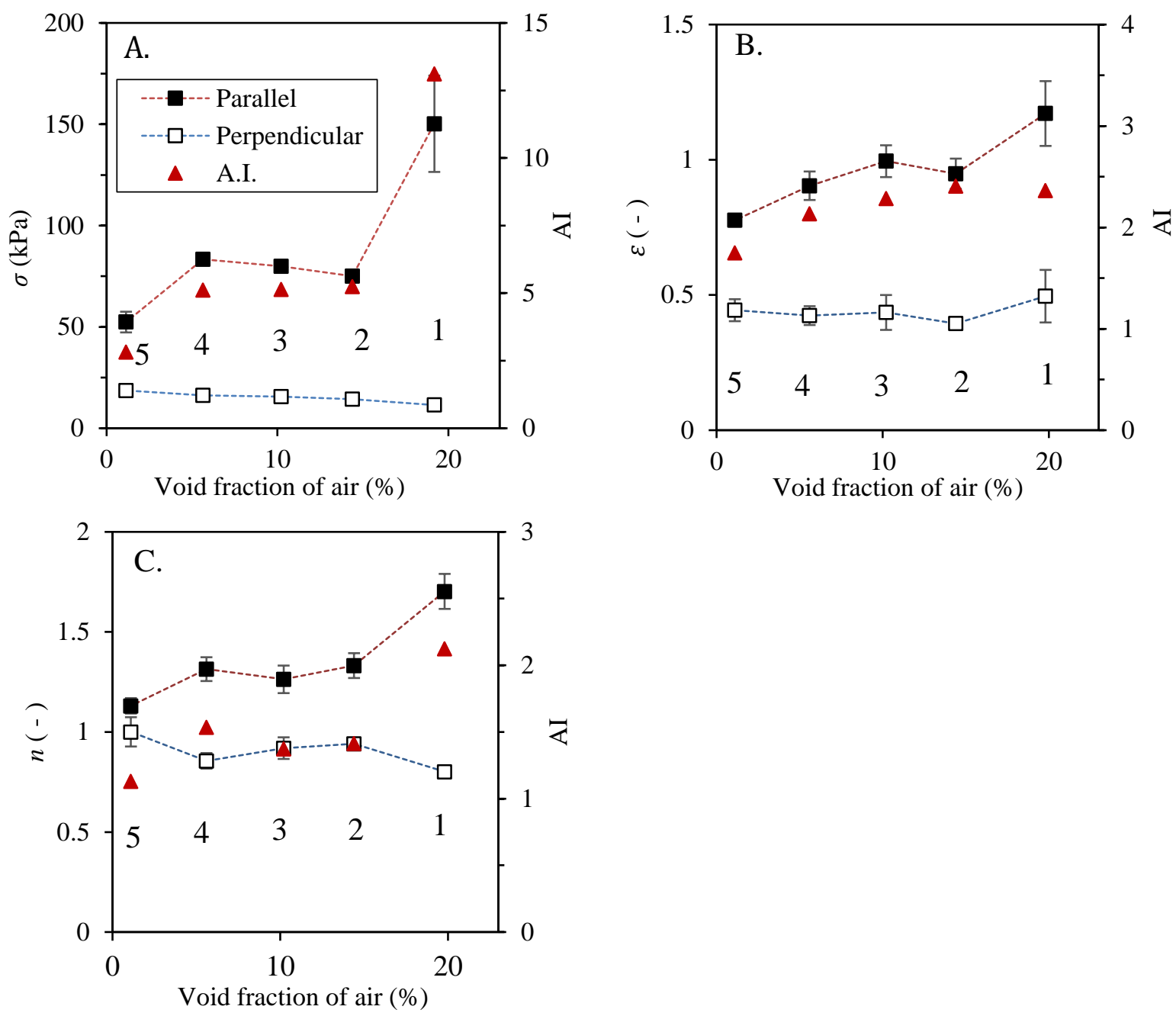

Fig. 2-5. Mechanical properties: (A) fracture stress $\sigma$, (B) fracture strain $\varepsilon$ and (C) apparent strain hardening coefficient $n$ as a function of void fraction of air for sheared 30 wt\% calcium caseinate materials 1-5, deformed parallel (filled squares) and perpendicular (open squares) to the shear direction. The error bars for the mechanical properties are \pm standard deviation; where not shown they were smaller than the marker used. The anisotropic index AI (red triangles) is ratio between the average data for the mechanical properties measured in the parallel and perpendicular directions to the shear flow.

Fig. 2-5C showed strain hardening in the parallel direction but not in the perpendicular direction in all materials. A similar behaviour was reported by Bast et al. (2015) for mozzarella cheese and Grabowska et al. (2014) for an anisotropic soy-gluten structure. The strain hardening behaviour in the calcium caseinate system became more pronounced when transglutaminase was added and air was incorporated. This strain 
hardening may be ascribed to the further alignment of the fibres around the bubbles, leading to an increase in strength. This only occurs in the parallel direction, not in the perpendicular direction. An increased crosslink density gives better adhesion between adjacent fibres and thus greater strength and better strain hardening (Kurtz et al., 1999). In the perpendicular direction, the fibres are just pulled apart. Here, the air bubbles work as a weak phase, similar to the role of the fat droplets in mozzarella cheese (Bast et al., 2015), which results in lower fracture strain and no strain hardening.

The sheared calcium caseinate material with entrapped air was more fibrous and exhibited larger anisotropy than the same material without air. We studied materials 2 and 5 further because these two have the largest and smallest void fraction and both were without transglutaminase (Figs. 2-6 and 2-7). In the parallel direction, material 2 reacts linearly elastic at small strains, followed by strain hardening and ultimately fluctuations close to the fracture point, indicating piecewise rupture of individual fibres or bundles of fibres. The strain hardening can be explained by further alignment of the fibres that are slightly misaligned due to the air bubbles. The fluctuating fracturing of material 2 is typical for fibrous composites, such as wood (Greil et al., 2002; Stanzl-Tschegg et al., 2011). Material 5, having little air, exhibited only very minor strain hardening and relatively smooth breakage (Fig. 2-7B), indicative of a relatively brittle material. In the perpendicular direction, both materials break at relatively low stresses with smooth breakage (Figs. 2-6 and 2-7C). 


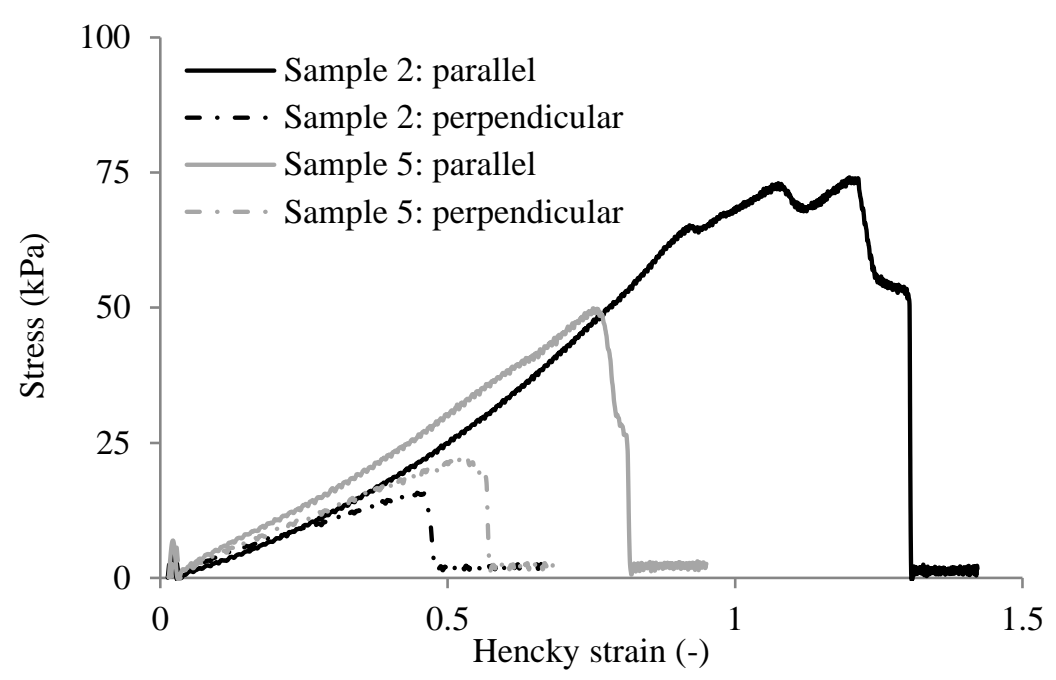

Fig. 2-6. Typical stress-strain curves of sheared $30 \mathrm{wt} \%$ calcium caseinate sample 2 and sample 5 in the parallel and perpendicular direction to the shear flow.

A.
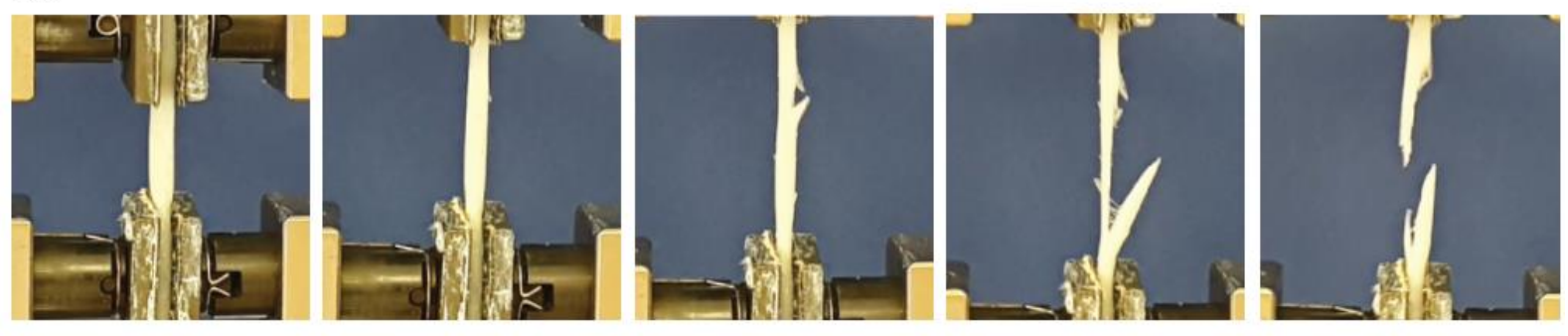

B.
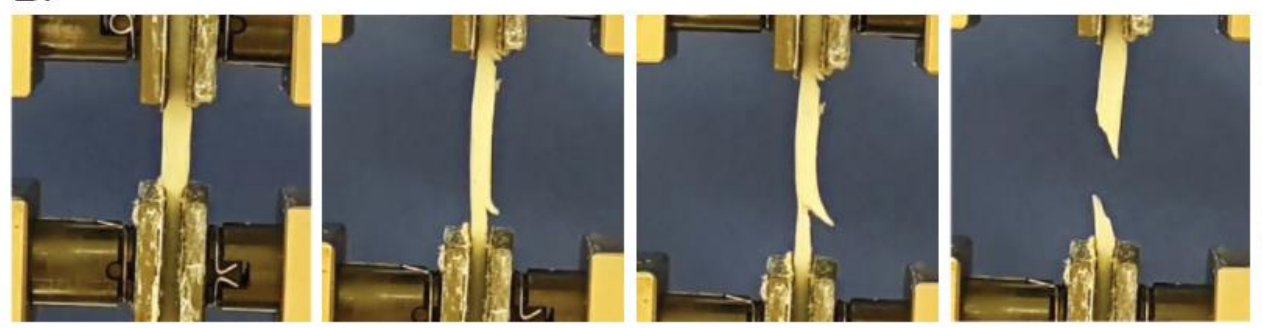

C.

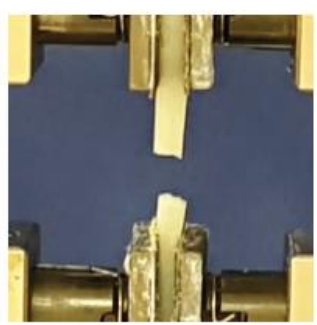

Fig. 2-7. Recorded images of a dog-bone specimen of samples 2 and 5 during the tensile test with a deformation rate of $3 \mathrm{~mm} \cdot \mathrm{s}^{-1}$. A: Sample 2 in the parallel direction. B: Sample 5 in the parallel direction. C: Sample 5 in the perpendicular direction to the shear flow.

\subsubsection{Relationship between microstructure and mechanical properties}

Fig. 2-8 interprets the influence of air bubbles in the matrix on the fracture behaviour in different orientations (Gibson and Ashby, 1997; Greil, Lifka, et al., 1998). Based on the tensile tests, we postulate that air bubbles act as a weak phase in the perpendicular direction. The bubbles facilitate widening of a crack in between fibres, which then 
propagates along the orientation of fibre. In the parallel direction, the incorporated air misaligns the fibres somewhat. Pulling then results in a realignment of the fibres along the axis of the tensile stress, resulting in a net stronger material under stress. The local stress accumulation that may result from any incipient crack is distributed over the whole surface area of the air bubble, and therefore the stress concentration lessens and the material thus is tougher (Vincent, 2011). This relies on the existence of relatively strong fibres and the adhesion between the fibres which also determines the overall strength. This is evident by the fact that there is still some minor anisotropy at a void fraction close to zero. The porosity makes the material less susceptible to failure in the parallel direction, and more susceptible to breakage in the perpendicular direction, as is known for composite materials.

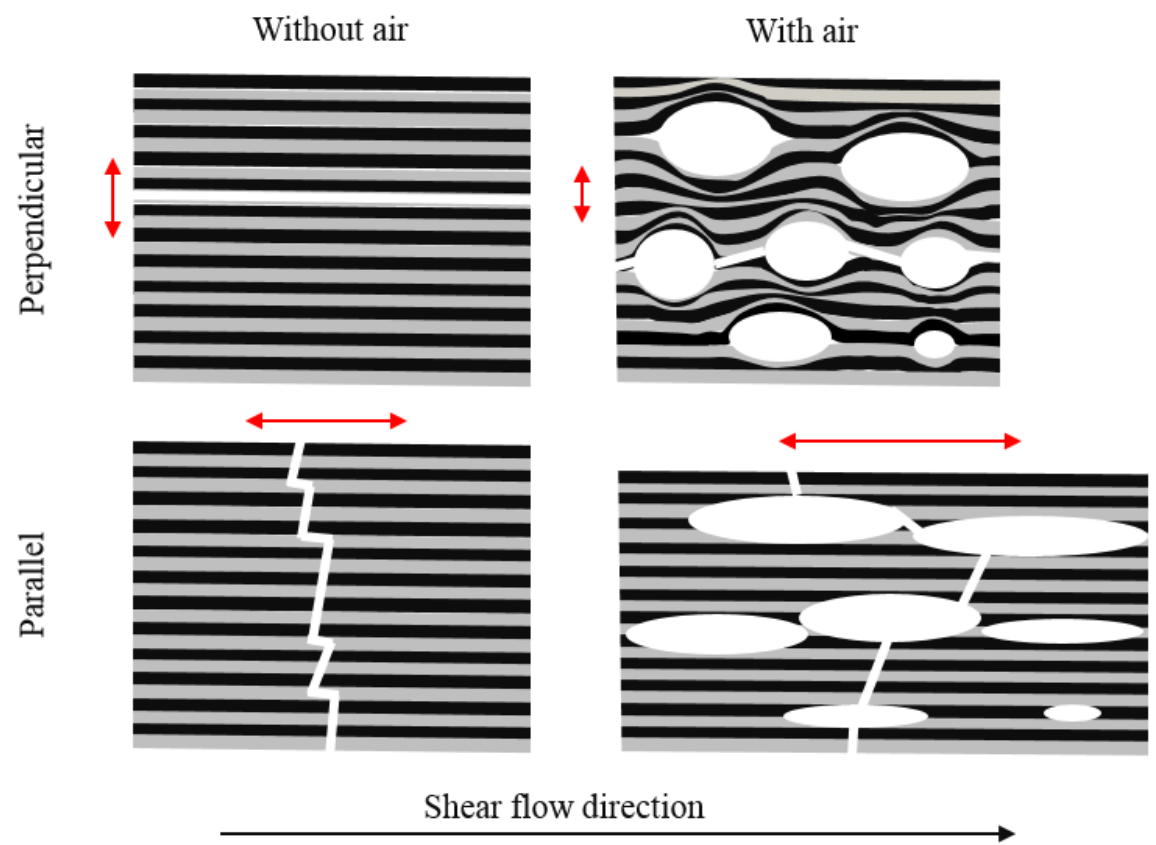

Fig. 2-8. Graphical illustration of the effect of air bubbles on the fracture behaviour in sheared calcium caseinate material. The red arrows represent the direction of applied tensile force. The white ellipses represents the air bubbles in the material and the white lines represents the crack paths. The dark and grey lines represent the shear-induced protein alignments. 
In the sheared calcium caseinate materials, the small air bubbles $\left(\leq 10^{-3} \mathrm{~mm}^{3}\right)$ were not deformed (-0.05-0.03), whereas larger air bubbles $\left(>10^{-3} \mathrm{~mm}^{3}\right)$ were slightly deformed (0.05-0.38). When comparing the air bubbles incorporated in calcium caseinate with a previous study on fibrous materials from soy protein isolate-pectin (Dekkers et al., 2018), we noticed that calcium caseinate materials entrap more air bubbles, which are smaller in size and less deformed than in soy protein isolate-pectin blends (0.33-0.56). This may be due to the amphiphilic nature of caseinate. Smaller air bubbles have more surface area, therefore cause more local misalignment of the fibres, and thus will cause more strain hardening and arresting of cracks. Indeed, we see this strain hardening and tougher materials in the parallel direction with caseinate, but not with soy protein isolate-pectin blends.

\subsection{Conclusion}

Air incorporated in a calcium caseinate dispersion enhances the macroscopic and mechanical anisotropy of the fibrous morphology formed after shearing. Air bubbles can be considered as an additional phase that influences the local alignment of fibres. Air incorporation causes the material to become tougher with strain hardening in the direction parallel to the fibres, but makes the material somewhat more susceptible to fracture in the perpendicular direction. This insight can be used to design materials with the right degree of anisotropy, by allowing the right amount of air to be incorporated into the material.

\section{Acknowledgements}

The authors would like to thank the China Scholarship Council (grant number 201406820015) for financial support, Friesland Campina for kindly supplying the protein ingredients, Marlies Nijemeisland from Delft University of Technology for helping with the light microscope images, Remco Hamoen for X-ray tomography and Jarno Gieteling for technical support. 


\section{Appendix 2A}

Table 2A-1 Mechanical properties of whipped sample 5.

\begin{tabular}{lccc}
\hline & $\begin{array}{c}\text { Fracture stress } \\
(\mathrm{kPa})\end{array}$ & $\begin{array}{c}\text { Fracture strain } \\
(-)\end{array}$ & $\begin{array}{c}\text { Strain hardening } \\
\text { coefficient }(-)\end{array}$ \\
\hline Parallel & $84.1 \pm 1.1$ & $1.02 \pm 0.07$ & $1.36 \pm 0.07$ \\
Perpendicular & $21.9 \pm 3.8$ & $0.62 \pm 0.09$ & $0.99 \pm 0.06$ \\
AI & 3.84 & 1.65 & 1.37 \\
\hline
\end{tabular}




\section{Chapter 3 .}

\section{Understanding the role of air and protein phase on mechanical anisotropy of calcium caseinate fibres}

This chapter has been published as Wang, Z., Tian, B., Boom, R., \& van der Goot, A. J. (2019). Understanding the role of air and protein phase on mechanical anisotropy of calcium caseinate fibres. Food Research International,121, 862-869. 


\begin{abstract}
Calcium caseinate dispersions can be transformed into anisotropic, fibrous materials using the concept of shear-induced structuring. The aim of this study is to further investigate the relative importance of air bubbles and protein on the mechanical anisotropy of calcium caseinate material. In this study, the effect of air on mechanical anisotropy of these fibrous materials was described with a load-bearing model, with the void fraction, and the bubble length and width as input parameters. The anisotropy of the protein phase was estimated using materials obtained from deaerated dispersions after shearing at different shear rates. We concluded that the deformation of air bubbles can only partly explain the mechanical anisotropy; the anisotropy of the protein phase is more important. Based on all results, we further concluded that the anisotropy of the protein phase was affected by the air bubbles present during the structuring process. This effect was explained by locally higher shear rate in the protein matrix during the structuring process.
\end{abstract}




\subsection{Introduction}

Dense calcium caseinate dispersions have been successfully transformed into a hierarchically fibrous structure using a process based on simple shear flow deformation (Manski et al., 2007a). Such fibrous material is structural and mechanical anisotropy. The presence of a pronounced fibrous structure is crucial for the development of an appealing meat analogue in terms of acceptability of consumers (Hoek et al., 2011). Therefore, calcium caseinate becomes an attractive material for the next generation of meat analogues.

The formation of calcium caseinate fibrous structure is based on the presence of caseinate micelles ( 100-200 $\mathrm{nm}$ ) in the dispersion. Further, these micelles exhibit mild adhesion due to the divalent $\mathrm{Ca}^{2+}$ (Manski et al., 2007a, 2007c). Upon shearing, those mildly adhesive micelles are aligned and concurrently fixated using transglutaminase. Recently, we found that the fibrous appearance and mechanical anisotropy of calcium caseinate materials are greatly enhanced by the presence of dispersed air bubbles $(\sim 100-400 \mu \mathrm{m})$ (Tian et al., 2018; Wang et al., 2019a). Several studies have showed that the geometrical anisotropy of air bubbles could result in the mechanical anisotropy of aerated materials (Cho and Rizvi, 2009; Griffiths et al., 2017; Hyun et al., 2001; Masmoudi et al., 2017; Nakajima, 2007). Therefore, it is interesting to quantify the effect of air bubbles on the mechanical anisotropy in calcium caseinate materials.

From a macroscopic viewpoint, the calcium caseinate fibrous material can be considered as a special foam in which air bubbles are dispersed within anisotropic protein matrices. Several empirical and theoretical models have been developed to predict the tensile properties of porous materials (Chen et al., 2013; Gibson and Ashby, 1997; Ji et al., 2006; Rice, 1996). Some of these models relate not only to total void fraction but also to the void geometry. For example, the load-bearing model is easily applied to idealized arrays of identically shaped and orientated voids (Rice, 1993). The load-bearing model assumes that the mechanical properties are determined by the minimum solid cross-sectional area parallel to the stress. In this approach, microstructural stress concentration effects are 
neglected (Rice, 1996). Hyun et al. (2001) showed that load-bearing model is suitable to determine the strength of porous copper. Recently, a model based on the cross-sectional area of a weak dispersed phase was modified to predict mechanical anisotropy of the fibrous soy protein isolate-pectin material (Dekkers et al., 2018).

In this study, we hypothesize that both the deformation of air and anisotropy of protein phase contribute to the macroscopic mechanical anisotropy. We apply the load-bearing model to quantify the effect of air in the calcium caseinate materials on their fracture properties. Both total void fraction and bubble geometry are considered. Fibrous materials were then prepared from aerated and from deaerated calcium caseinate dispersions using different shearing rates. Deaeration in these concentrated dispersions was achieved by heating, centrifuging and application of a long rehydration time before shearing (Wang et al., 2019a). The geometry of the air bubbles in the final products was studied with X-ray tomography, while the directional tensile strengths were determined using a texture analyser. Finally, the predicted mechanical anisotropy from the loadbearing model was compared to the mechanical anisotropy found experimentally.

\subsection{Material and methods}

\subsubsection{Material}

Spray dried calcium caseinate was kindly provided by DMV International (Veghel, the Netherlands). This calcium caseinate contained $92 \mathrm{wt} \%$ protein and $1.46 \mathrm{wt} \%$ calcium according to the manufacturer's specifications. The dry matter was 94.82 wt\%. Weight was given as powder weight.

\subsubsection{Protein dispersions preparation for preparative shearing}

An aerated protein dispersion was prepared by manually mixing 30 wt\% calcium caseinate powder in demineralized water ( $\mathrm{pH}$ 6.7-6.9) with a spatula for 1 min. Deaeration was achieved through heating the dispersion at $80^{\circ} \mathrm{C}$ for $5 \mathrm{~min}$, followed by centrifuging ( $2500 \times g$ for $2 \mathrm{~min})$ and subsequently leaving it at room temperature for $55 \mathrm{~min}$. 


\subsubsection{Material preparation in the shear cell device}

Well-defined shear flow was applied using a custom built shearing device (Wageningen University, the Netherlands), which consists of a rotating plate (the bottom cone, angle $\alpha_{\text {plate }}=105^{\circ}$ ) and a stationary cone (the top cone, angle $\alpha_{\text {cone }}=100^{\circ}$ ). Further details are described in an earlier publication (van der Zalm et al., 2012). The cones were temperature controlled with a heating and a cooling water bath. After preparation, the dispersion was immediately transferred to the pre-heated $\left(50^{\circ} \mathrm{C}\right)$ device. Dispersions were then subjected to shearing at various rates $(0,3,10,25,50,75,100$ or $150 \mathrm{rpm})$ for $5 \mathrm{~min}$. After processing, the material was cooled for $10 \mathrm{~min}$ at $4^{\circ} \mathrm{C}$. Tensile tests were performed within $1 \mathrm{~h}$ after removing the sample from the shear cell device. Part of the materials was stored at $-20^{\circ} \mathrm{C}$ prior to further analysis. The preliminary results when using shear rates higher than $150 \mathrm{rpm}$ were shown to be not reliable due to the instability of the inner cone during shearing.

\subsubsection{Tensile strength analysis}

A Texture Analyser (Instron Testing System, table model type 5564) was used with a load cell of $100 \mathrm{~N}$. The material that was not sheared (referred to as $0 \mathrm{rpm}$ ) was too weak to measure its tensile strength. From the other materials, dog-bone shaped pieces were cut with a length of $15.2 \mathrm{~mm}$ and a width of $3.18 \mathrm{~mm}$ using a tensile bar. The thickness of the samples varied between 4 and $6 \mathrm{~mm}$. The samples were taken parallel and perpendicular to the shear flow (based on the shear-vorticity plane). The tensile tests were conducted at a constant deformation speed of $3 \mathrm{~mm} \cdot \mathrm{s}^{-1}$. Grips with abrasive paper were used to prevent slip during testing. At least three specimens per direction were measured per sample, and three samples per condition were tested. The results obtained from these tensile tests were depicted as force-displacement curves. Blue Hill software was used to determine a fracture point and to calculate the fracture stress $(\sigma, \mathrm{kPa})$ and fracture strain $(\varepsilon,-)$.The ratio of the mechanical properties between the parallel and perpendicular directions to the shear flow was used as an index for the mechanical anisotropy of the samples. 


\subsubsection{X-ray tomography}

Samples (roughly $3 \times 4 \times 20 \mathrm{~mm}$ ) were scanned using a GE Phoenix v|tome $\mid \mathrm{x} \mathrm{m}$ tomographer (General Electric, Wunstorf, Germany) set at $80 \mathrm{kV} / 90 \mu A$. The system contains two X-ray sources; the $240 \mathrm{kV}$ micro-focus tube with a tungsten target was used. The images were recorded by a GE DXR detector array with $2024 \times 2024$ pixels (pixel size, $200 \mu \mathrm{m}$ ). The detector and object were located $815 \mathrm{~mm}$ and $28.55 \mathrm{~mm}$ from the Xray source, respectively. This results in a spatial resolution of $7.00 \mu \mathrm{m}$. A full scan consisted of 750 projections over $360^{\circ}$. Four images were taken for each projection. The first image was skipped because it probably contained information from the previous projection; images 2-4 were averaged into one projection. GE reconstruction software (Wunstorf, Germany) was used to calculate the 3D structure via back projection. The 3D images were analysed using Avizo imaging software version 9.3.0. The analysis included the total volume, total air volume in the sample, void fraction of air $(p)$, the length of air bubble parallel to the shear flow $(a)$ and the average width of the air bubble perpendicular to the shear flow ( $b$, based on velocity gradient-shear flow plane and vorticity-shear flow plane).

\subsubsection{Reflective light microscopy}

A fresh sample (50 rpm) was stretched by hands in the parallel direction to shear flow. The resulting deformed material was then observed with a digital microscope (Smartzoom 5) with 50× magnification.

\subsection{Theory}

Based on load-bearing model, the tensile stress of porous material is given as follows:

$$
\sigma=\sigma_{c} \cdot \varphi
$$

Where $\sigma_{c}$ is the theoretical fracture stress in the same material without air; $\varphi$ is the minimum cross-sectional area fraction of continuous phase. We simplify calcium caseinate material by considering the dispersed air bubbles as many small cubes inside a larger cube (h) (Fig. 3-1). We assume that all these cubes have a similar size and are 34 
deformed in a similar manner. The deformation will result in one dimension becoming longer $(a)$, and we assume that the volume remains constant, and the two other dimensions will remain the same $(b)$. Then,

$$
p=\frac{\mathrm{a} \cdot b^{2}}{h^{3}}
$$

When stretching the material in the parallel direction of the shear flow (Fig. 3-1A), the minimum cross-sectional area fraction of the air bubbles is given by:

$$
\varphi_{\|}=1-\frac{b^{2}}{\mathrm{~h}^{2}}=1-\left(\frac{\mathrm{a}}{b}\right)^{-\frac{2}{3}} \cdot p^{\frac{2}{3}}
$$

When stretching the material in the perpendicular direction of the shear flow (Fig. 3-1B), the minimum cross-sectional area fraction of the air bubbles is given by:

$$
\varphi_{\perp}=1-\frac{\mathrm{a} \cdot b}{\mathrm{~h}^{2}}=1-\left(\frac{\mathrm{a}}{b}\right)^{\frac{1}{3}} \cdot p^{\frac{2}{3}}
$$

A.

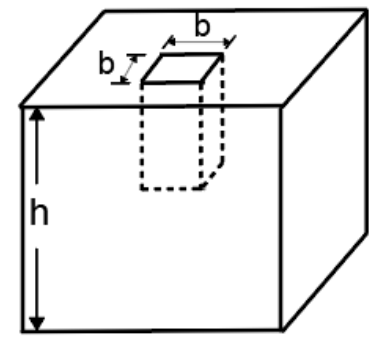

B.

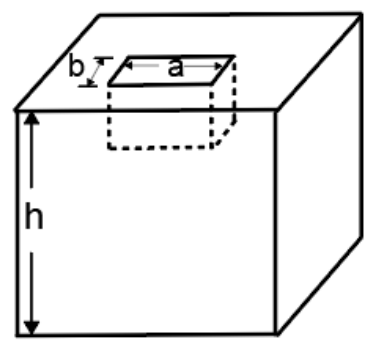

Fig. 3-1. Schematic of the load-bearing area model for calcium caseinate material with cuboid void: deformation (shear flow) direction parallel to the tensile stress (A); deformation (shear flow) direction perpendicular to the tensile stress (B).

Combining Eqs. (3-3) and (3-4) into Eq. (3-1) yields:

$$
\begin{aligned}
& \sigma_{\|}=\sigma_{c \|} \cdot\left(1-\left(\frac{\mathrm{a}}{b}\right)^{-\frac{2}{3}} \cdot p^{\frac{2}{3}}\right) \\
& \sigma_{\perp}=\sigma_{c \perp} \cdot\left(1-\left(\frac{\mathrm{a}}{b}\right)^{\frac{1}{3}} \cdot p^{\frac{2}{3}}\right)
\end{aligned}
$$


It should be noted that the values of $\sigma_{c \perp}$ and $\sigma_{c \|}$ are only the same in a matrix that is homogeneous (i.e. isotropic). In our case, the matrix consists of sheared calcium caseinate, which might be anisotropic. This explains why different symbols were taken to include anisotropy effect in Eqs. (3-5) and (3-6).

We define the ratio between the fracture stress in the parallel and perpendicular directions as the anisotropy index $(A I)$, which can be expressed as

$$
A I=\frac{\sigma_{\|}}{\sigma_{\perp}}=\frac{\sigma_{c \|}}{\sigma_{c \perp}} \cdot \frac{1-\left(\frac{\mathrm{a}}{b}\right)^{-\frac{2}{3}} \cdot p^{\frac{2}{3}}}{1-\left(\frac{\mathrm{a}}{b}\right)^{\frac{1}{3}} \cdot p^{\frac{2}{3}}}=A I_{\text {protein }} \cdot A I_{\text {air }}
$$

Thus, the anisotropy index of fracture stress $A I$ is equal to the product of the anisotropy index of continuous phase $A I_{\text {protein }}$ and the anisotropy index of the deformed air bubble $A I_{\text {air }}$

Fig. 3-2A demonstrates the effect of air holdup according to this model on the tensile stress in two directions, given a constant void fraction $(p=0.2)$ and assuming an isotropic continuous phase $\left(\sigma_{c \|}=\sigma_{c \perp}=100 \mathrm{kPa}\right)$. The fracture stress increases in the parallel direction with increased deformation of the air bubbles, and it decreases in the perpendicular direction. Consequently, the values of $A I$ increases with increased deformation of the air bubbles. When it is assumed that the continuous phase is anisotropic $\left(\sigma_{c \|}=100 \mathrm{kPa} ; \sigma_{c \perp}=50 \mathrm{kPa}\right)$, the value of $A I$ is twice compared to the material with an isotropic continuous phase. The $A I$ can thus result from a combination of anisotropic protein phase and anisotropy originating from deformed air bubbles. 

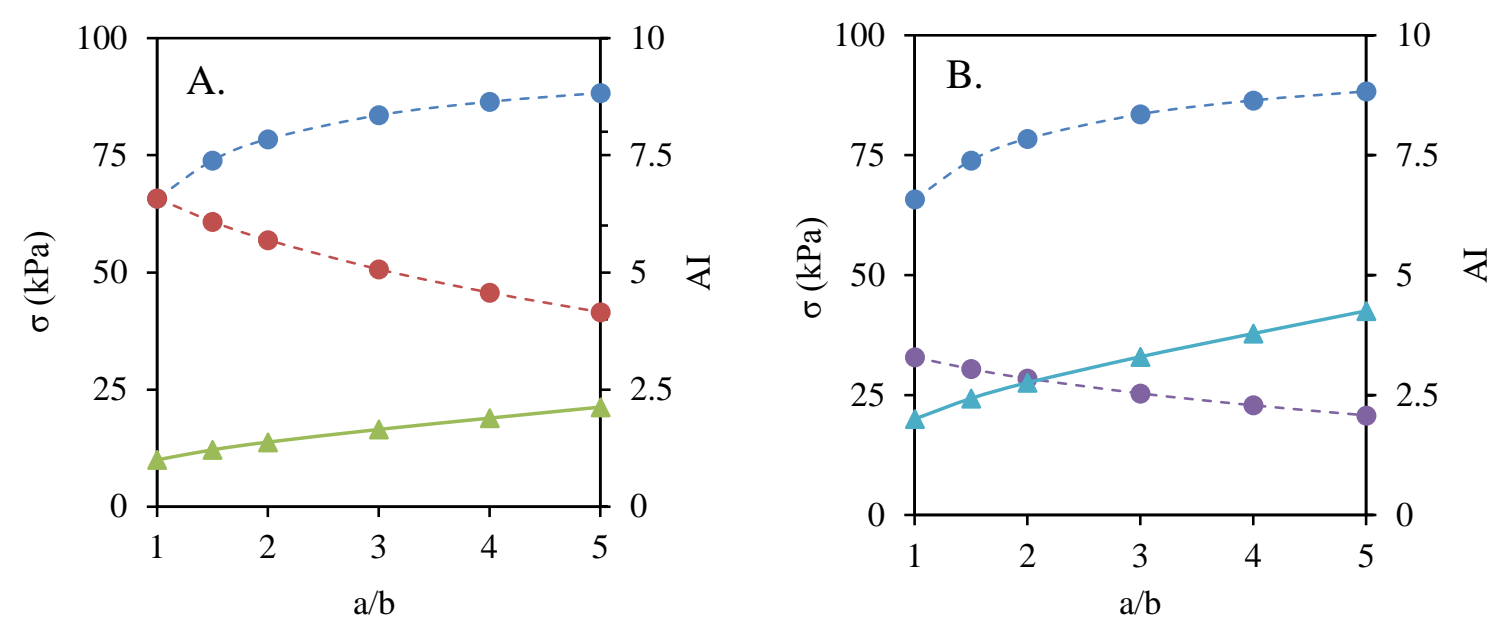

Fig. 3-2. A: Calculation of the fracture stress in the parallel $(\bigcirc)$ and perpendicular $(\bigcirc)$ direction and anisotropy index $A I(\Delta)$, while keeping a constant porosity $(p=0.2)$ and assuming an isotropic continuous phase $\left(\sigma_{c \|}=\sigma_{c \perp}=100 \mathrm{kPa}\right)$; B: calculation of fracture stress in the parallel $(\odot)$ and perpendicular $(\odot)$ direction and anisotropy index $A I(\Delta)$, while keeping a constant porosity $(p=0.2)$ and assuming an anisotropic continuous phase $\left(\sigma_{c \|}=100 \mathrm{kPa} ; \sigma_{c \perp}=50 \mathrm{kPa}\right)$. The lines have been added to guide the eye. 


\subsection{Results}

\subsubsection{Structure formation from aerated calcium caseinate}

Aerated calcium caseinate dispersions (around $20.0 \mathrm{v} \%$ of air), were sheared at different shear rates (0-150 rpm, $5 \mathrm{~min})$. Visual inspection of the resulting materials revealed clear differences. Without shear (0 rpm), the material was a weak, homogeneous gel without fibres or layers. The material that was sheared at $3 \mathrm{rpm}$ was weak and easily broken when discharged from the device (Fig. 3-3A). This material was anisotropic but did not contain fibres. The applications of a shear rate of $10 \mathrm{rpm}$ or $25 \mathrm{rpm}$ resulted in some thick fibres upon tearing the materials manually. Further increasing the shear rate to 50, 75 and 100 rpm resulted in pronounced fibrous materials (Fig. 3-3B). The material made at $150 \mathrm{rpm}$ showed less fibres upon tearing compared to the material sheared at 50, 75 and $100 \mathrm{rpm}$.
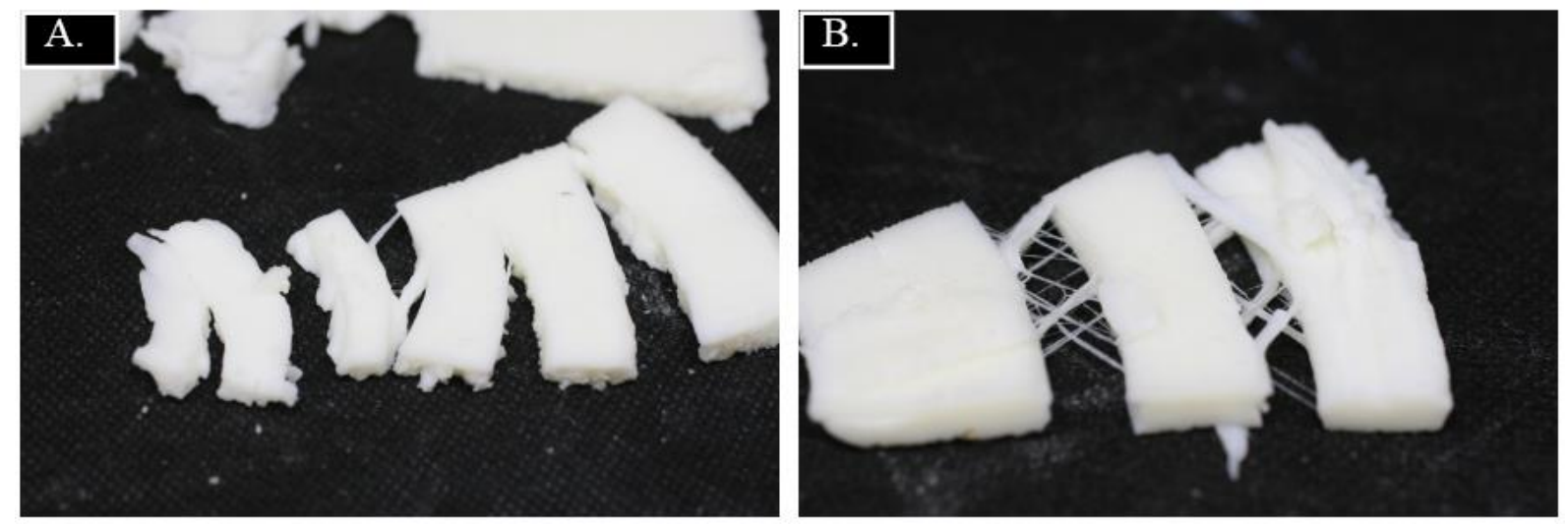

Fig. 3-3. Macrostructure of $30 \mathrm{wt} \%$ aerated calcium caseinate material sheared at $3 \mathrm{rpm}$ (A) and $75 \mathrm{rpm}$ (B). The length of images is approximately $4-7 \mathrm{~cm}$.

The geometries of the aerated and sheared materials (Fig. 3-4) were analysed using 2D and reconstructed 3D X-ray tomography (XRT). Without shearing ( $0 \mathrm{rpm})$, the air was randomly distributed throughout the protein matrix. Shearing calcium caseinate dispersion at $3 \mathrm{rpm}$ resulted in slightly deformed air bubbles. This deformation increased with increasing shear rate, while the total air holdup clearly decreased when sheared at $150 \mathrm{rpm}$. In the latter material, the air was mostly located near the inner or stationary cone (left side of the 2D image), and only small air bubbles remained closer to the rotating 
outer cone (right side of the 2D image). This suggests that the larger air bubbles were expelled from the rotating outer cone during shear.

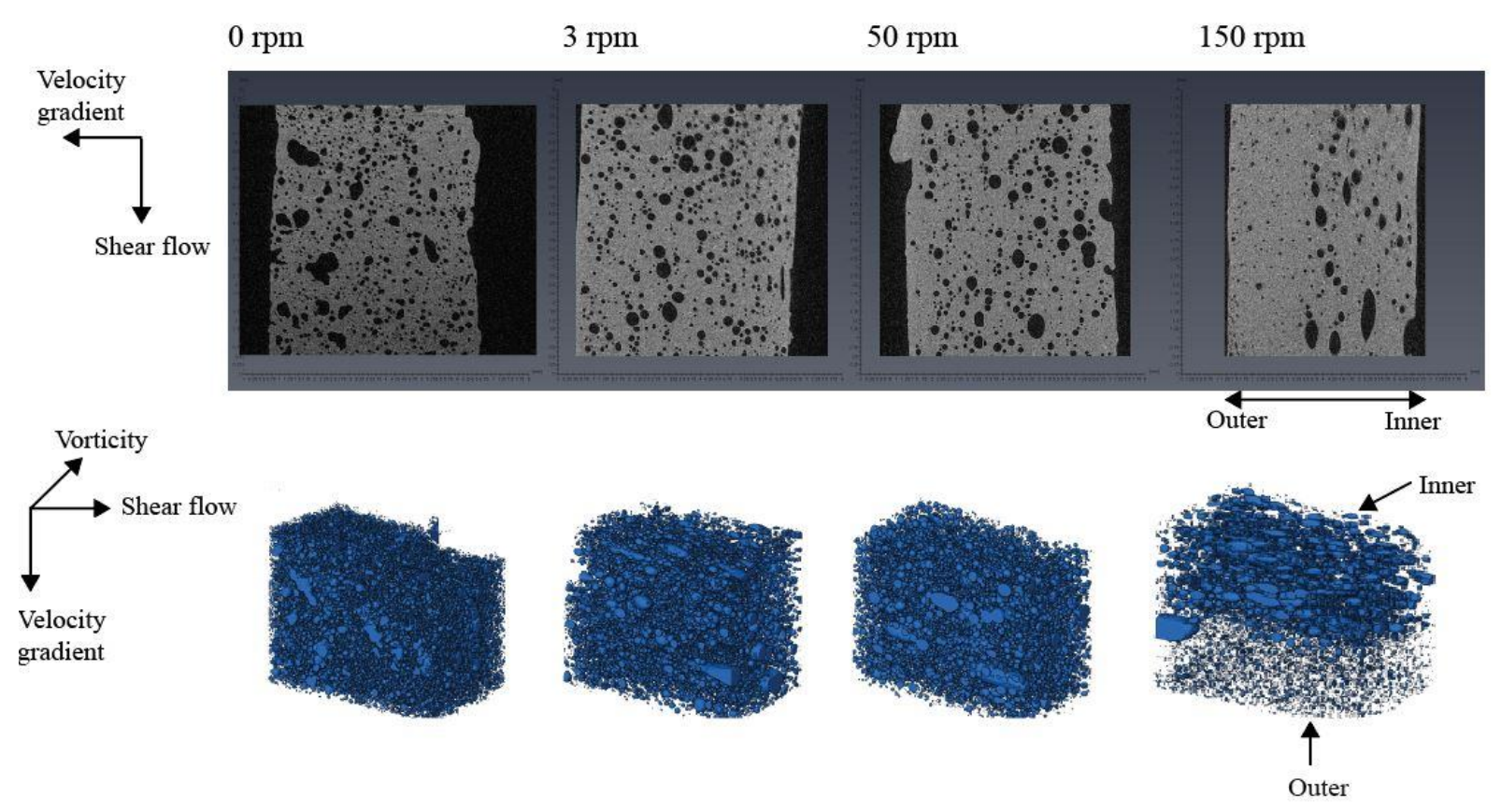

Fig. 3-4. X-ray tomography images of air bubbles in calcium caseinate material that were sheared at various shear rate $(0,3,50$ and $150 \mathrm{rpm})$ for $5 \mathrm{~min}$.

Shearing at different rates (0-150 rpm) affected both the total air holdup and the deformation of the air bubbles in the material (Fig. 3-5). Without shearing (0 rpm), the material contained $20.0 \mathrm{v} \%$ air, while visual observations showed hardly any bubble deformation. Fig. 3-5A shows that shearing reduced the total air holdup (variation between 13.6 and $15.1 \mathrm{v} \%$ ) up to a shear rate of $25 \mathrm{rpm}$, which was mainly due to the loss of the smallest $\left(<10^{-3} \mathrm{~mm}^{3}\right)$ and the largest air bubbles $\left(>10^{-1} \mathrm{~mm}^{3}\right)$. The material sheared at $50 \mathrm{rpm}$ contained the highest void fraction of $17.5 \mathrm{v} \%$ air among the sheared materials (but still lower than the material without shearing). At higher shear rates (75$150 \mathrm{rpm}$ ), the materials contained less air. Fig. 3-5B shows that the shear deforms air bubbles when their volumes are larger than $10^{-3} \mathrm{~mm}^{3}$. However the deformation did not strongly change when varying the shear rate between 3 and $75 \mathrm{rpm}$. The large air bubbles $\left(>10^{-1} \mathrm{~mm}^{3}\right.$ ) were mostly deformed when processing at 100 and $150 \mathrm{rpm}$. It is possible that shear force had an influence on the external forces that are resulting in deformation, 
breakup and coalescence of air bubbles. To summarize, overall air holdup decreased with higher shear rates, whereas the deformation degree of air bubbles was not strongly influenced by the shear rate.
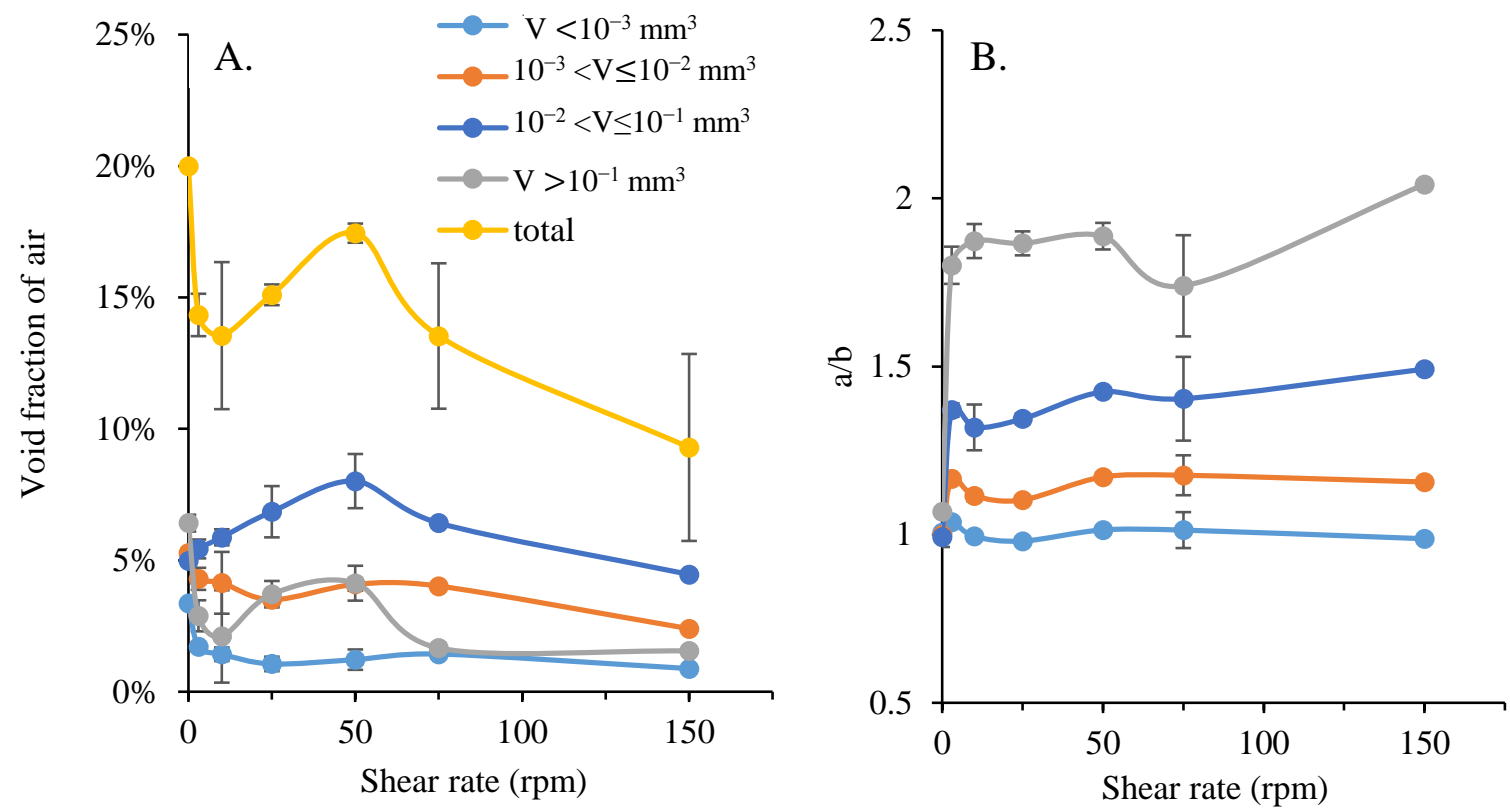

Fig. 3-5. X-Ray tomography analysis of air bubbles when varying the shear rate for $30 \mathrm{wt} \%$ calcium caseinate materials: $(\mathbf{A})$ overall void fraction and the void fraction at different volume ranges; (B) the ratio between long and short axis of the air bubbles (a/b) at different volume ranges. The error bars are \pm standard deviation; where not visible, error bars were smaller than the marker used.

Fig. 3-6 summarizes the effect of shear rate on the fracture mechanics of the materials. In the absence of shear, the material was too weak to perform a tensile test. The fracture stress and strain increased with increasing shear rate (3-75 rpm) in the parallel direction. Shearing at $3 \mathrm{rpm}$ resulted in only slight anisotropy. Shearing at 10 and $25 \mathrm{rpm}$ resulted in more pronounced anisotropy. These materials had a higher fracture stress parallel to the shear flow, and a lower fracture stress perpendicular to the shear flow when compared to the material that was sheared at $3 \mathrm{rpm}$. Materials sheared at 50, 75 and 100 rpm had most pronounced anisotropy. Their fracture stress and strain were the highest in the parallel direction. The slight increase of the fracture stress in the perpendicular direction from 50 to $150 \mathrm{rpm}$ can be explained by the decreasing of the air holdup. The 
material sheared at $150 \mathrm{rpm}$ was much weaker compared to the materials sheared at 50$100 \mathrm{rpm}$ in the parallel direction. The explanation could be that a high shear rate leads to break up the protein alignment.
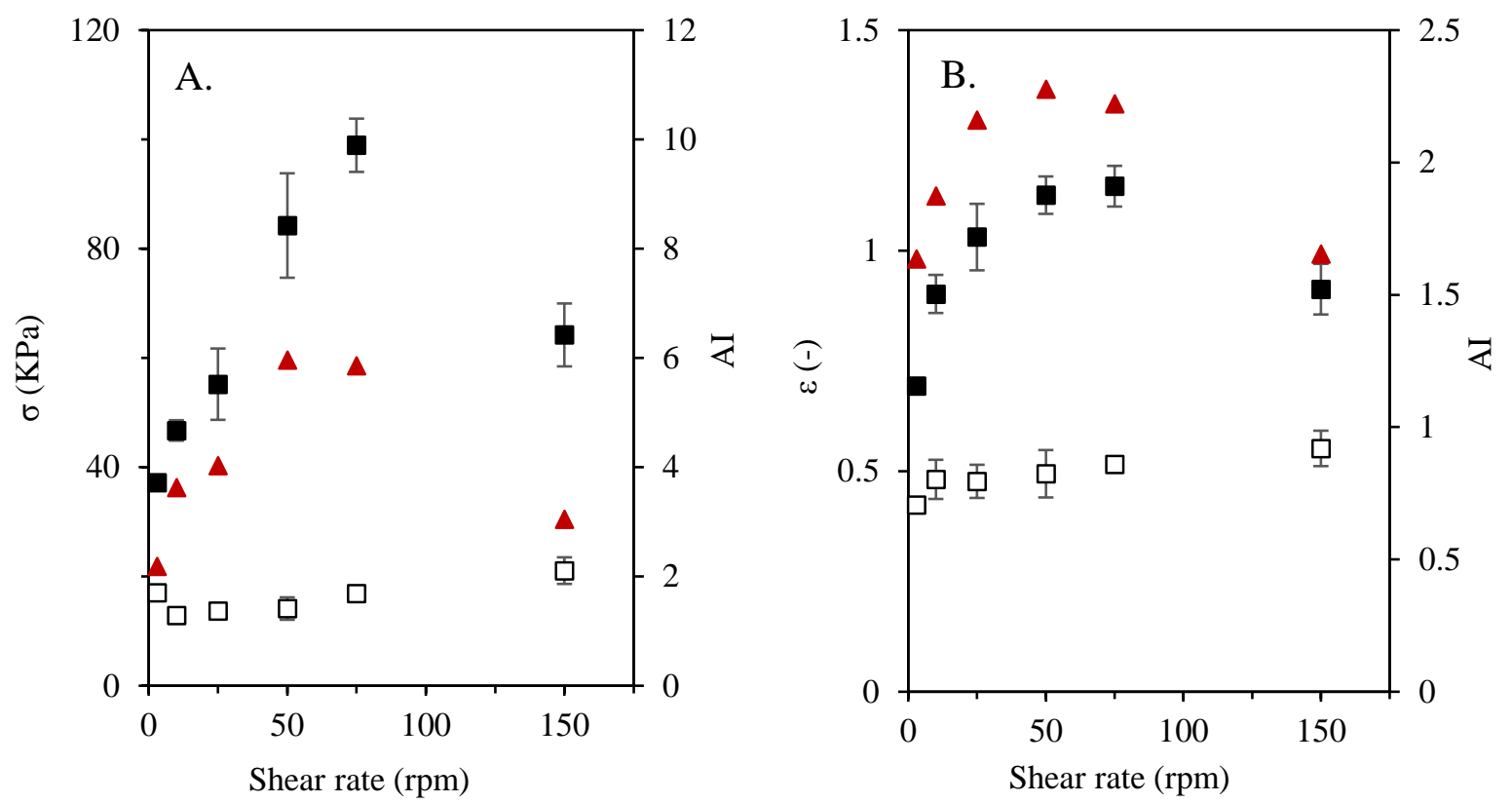

Fig. 3-6. Measured mechanical properties: (A) fracture stress $\sigma$ and (B) fracture strain $\varepsilon$ of $30 \mathrm{wt} \%$ calcium caseinate materials as a function of shear rate $(3,10,25,50,75,100$ and $150 \mathrm{rpm}$ ) parallel ( $\mathbf{\square})$ and perpendicular $(\square)$ to the shear direction. The error bars are \pm standard deviation; where not visible, error bars were smaller than the marker used. The anisotropy index $(\boldsymbol{A})$ is the ratio between the mechanical properties measured in the parallel and perpendicular directions.

\subsubsection{Structure formation from deaerated calcium caseinate dispersions}

In the absence of air bubbles, the $30 \mathrm{wt} \%$ calcium caseinate dispersions formed materials with different properties. Shearing at low rates $(3,10$ and $25 \mathrm{rpm})$ resulted in isotropic materials accompanied by some expelled water and subtle syneresis (Fig. 3-7A). Further, the applied shear rates were too low to create anisotropy (Manski et al., 2008). Materials with an anisotropic structure were formed at higher shear rates (40-75 rpm). A further increase of the shear rate $(100-150 \mathrm{rpm})$ resulted in fibres that were only visible upon tearing manually. Fig. 3-7C showed that the anisotropy index of fracture stress increased 
with increasing shear rate (40-150 rpm). A linear relationship was observed between the anisotropy index of fracture stress and shear rate with an intercept of 1 and a 95\% confidence interval from 0.0248 to 0.0290 . This empirical equation in Fig. 3-7C can be used to estimate the "theoretical" mechanical anisotropy index of the deaerated dispersions sheared at low rates (3-25 rpm).

A.

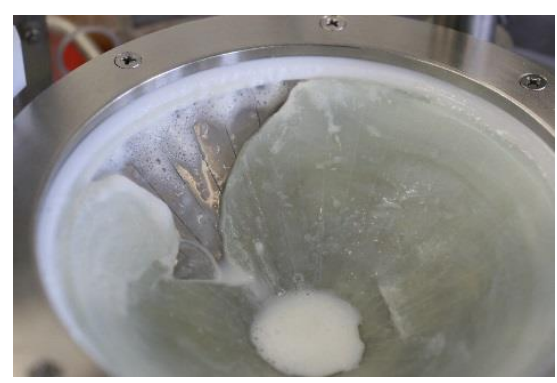

B.

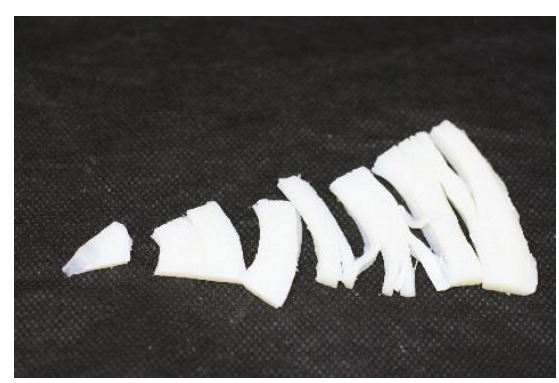

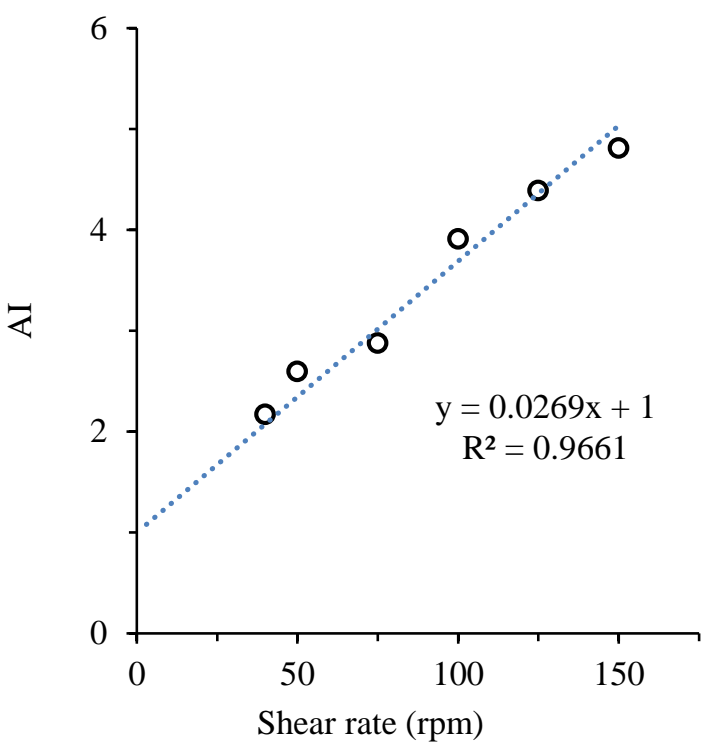

C.

Fig. 3-7. Macrostructure of sheared $30 \mathrm{wt} \%$ deaerated calcium caseinate material at $3 \mathrm{rpm}$ (A) and $100 \mathrm{rpm}$ (B). Anisotropy index of fracture stress for $30 \mathrm{wt} \%$ deaerated calcium caseinate materials as a function of shear rate $(40,50,75,100,125$ and $150 \mathrm{rpm})$. The dotted line represents the fit.

\subsection{Discussion}

The sheared calcium caseinate materials are known to create an anisotropic structure. However, we identified the importance of an additional phase that also contributes to the material properties: the air phase (Wang et al., 2019a). The importance of air is based on the large differences in the structural and mechanical anisotropy between the aerated and non-aerated materials, and the slight deformation of the air bubbles. However, anisotropy was also observed in the non-aerated materials, which means that the protein phase itself can become anisotropic as well. We therefore propose that both the protein matrix and the dispersed air bubbles contribute to the mechanical anisotropy in the material. 
The X-ray tomography measurements provide the parameters needed to describe the change in mechanical properties due to the presence of air bubbles using the model. Those parameters are the volume fraction of the air $p$, the average length of the bubbles $(a)$ parallel to the shear and the average width $(b)$ perpendicular to the shear direction. With that, we calculate the fracture stress in the parallel $\left(\sigma_{\|}\right)$and the perpendicular $\left(\sigma_{\perp}\right)$ directions as well as the anisotropy index $\left(A I_{\text {air }}\right)$ of the fracture stress using Eqs. (3-5), (3-6) and (3-7).

One should bear in mind that the material will be extended during the tensile test in the parallel direction. This extension of the material in the direction of the shear flow will also extend the bubble (Fig. 3-8). To include this effect, Eq. (3-5) is modified:

$$
\sigma_{\|}=\sigma_{c \|} \cdot(1-p)
$$

As a result, Eq. (3-7) can be expressed as:

$$
A I_{\text {air }}=\frac{1-p}{1-\left(\frac{\mathrm{a}}{b}\right)^{\frac{1}{3}} \cdot p^{\frac{2}{3}}}
$$

assuming an isotropic protein phase $\left(\sigma_{\mathrm{c \|}}=\sigma_{\mathrm{c \perp}}\right)$. The mechanical anisotropy caused by the deformation of air bubbles is plotted as a function of the shear rates applied when making the materials (Fig. 3-9). Eq. (3-7) predicts only little anisotropy, given that the bubbles only show limited deformation. However, the bubble deformation during the actual tensile test is much larger, which is illustrated in Fig. 3-8. If we use the deformations obtained just before breakage (Fig. 3-8F), the prediction of the mechanical anisotropy is still much lower than what is found with experimental tensile tests. 

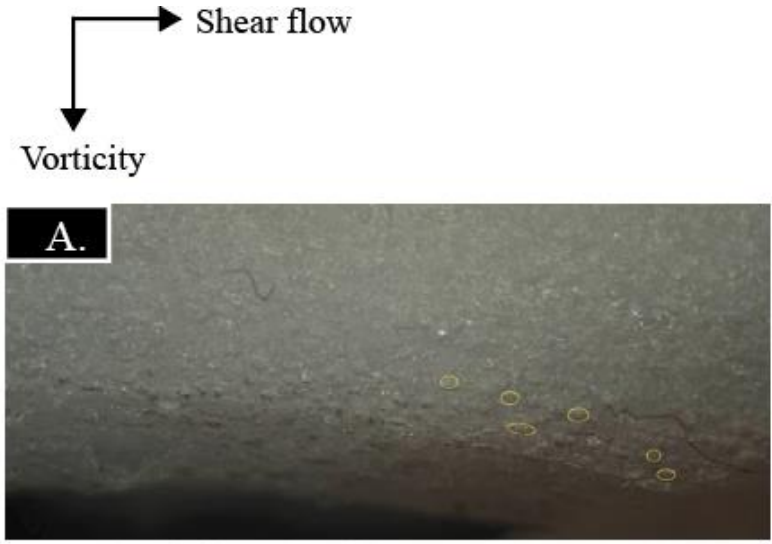

\section{C.}

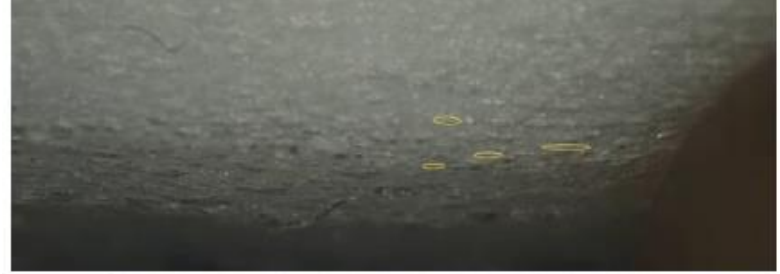

E.

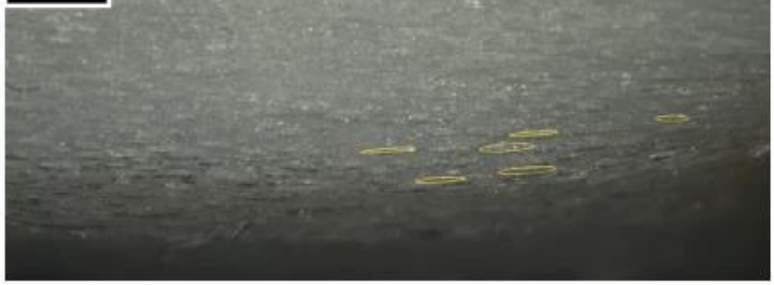

B.

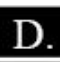

F.

Fig. 3-8. Morphology changes of air bubbles in a fresh sample (50 rpm, $5 \mathrm{~min}$ ) when stretched by hand in the parallel direction to shear flow. A: the bubble deformation before stretching; B-E: the bubble deformation upon stretching; F: the bubble formation upon stretching just before breakage. The yellow ellipses have been drawn to sharpen the morphology of bubbles.

Fig. 3-9 depicts the experimental mechanical anisotropy index of fracture stress for the aerated materials and the predicted anisotropy index based on the deformation of air bubbles as a function of the shear rate. Clearly, the deformation of air bubbles does not fully account for the observed mechanical anisotropy. As a consequence, part of the anisotropy must be caused by anisotropy within the protein phase itself. Indeed, a linear relationship between the anisotropy index of deaerated dispersions and shear rate (40$150 \mathrm{rpm}$ ) was found (Fig. 3-7). In addition, we used Eqs. (3-6) and (3-8) to estimate the fracture stress of the protein matrix in the aerated materials and compared with the experimental data of deaerated materials (Fig. 3-10A, B). The estimated data was in good 
agreement with the experimental data in the perpendicular direction (Fig 3-10B). However, the predicted values were higher than the experimental values in the parallel direction. As a result, the experimental anisotropy indices are higher than the experimental values of deaerated material (3-100 rpm) (Fig. 3-10C). Only the estimation of $150 \mathrm{rpm}$ was much lower than that of $100 \mathrm{rpm}$. This is thought be due to the breakup of protein alignment in the aerated material. Thus, Eq. (3-8) is not suitable for the calcium caseinate material with high shear rate (above $100 \mathrm{rpm}$ ).

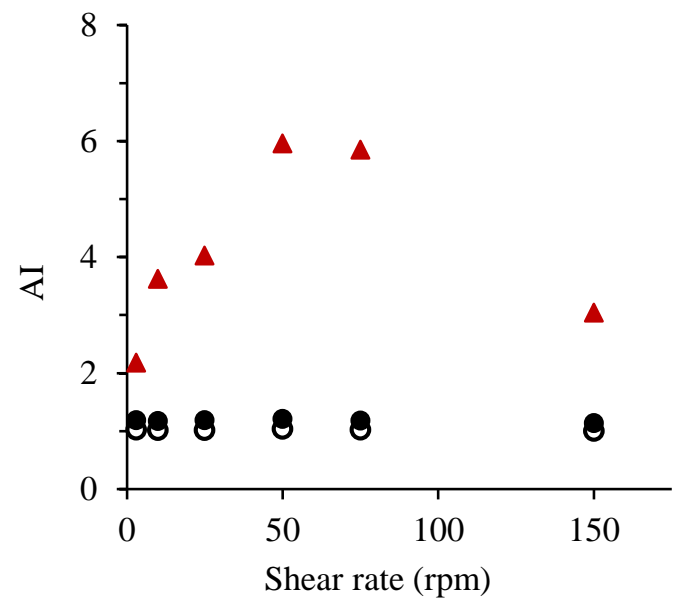

Fig. 3-9. The experimental mechanical anisotropy index ( $\Delta)$ of fracture stress for the aerated materials at various shear rate (3-150 rpm) for $5 \mathrm{~min}$; the predicted anisotropy index based on the bubble deformation before stretching $(\mathrm{O})$, and the bubble deformation upon stretching just before breakage $(\mathbf{O})$.
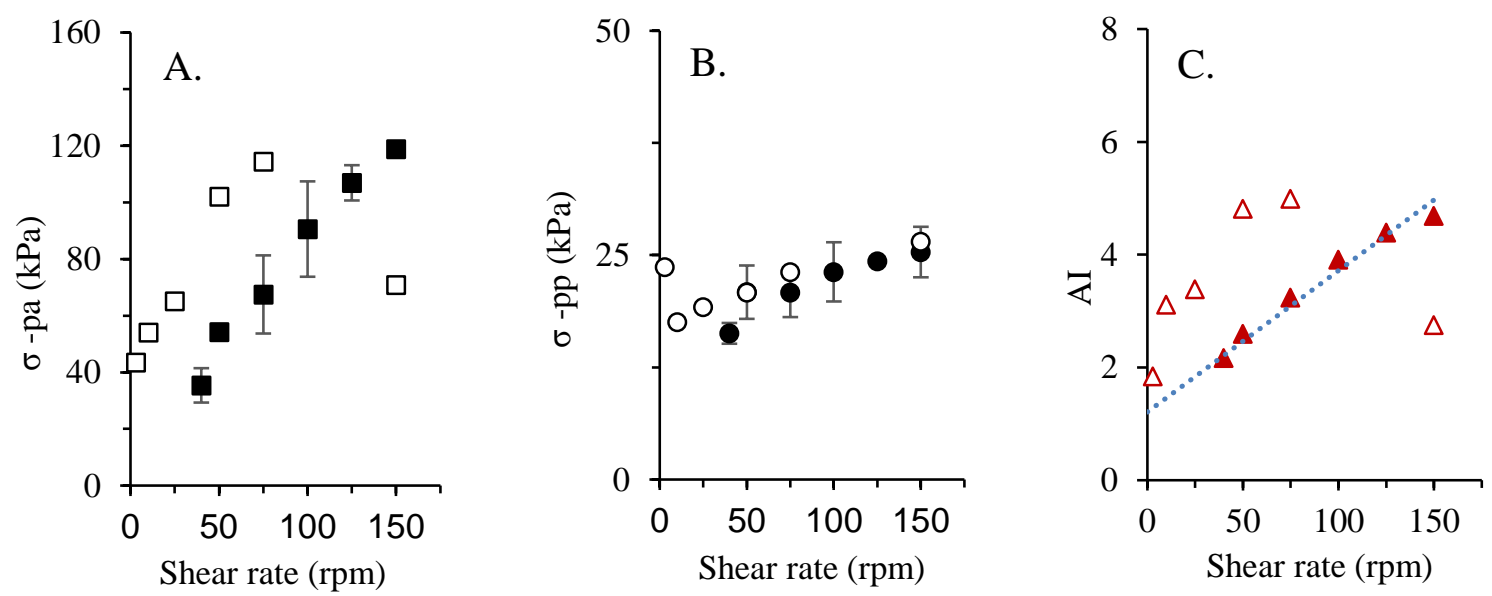

Fig. 3-10. Comparing experimental (closed symbol) with estimated (open symbol) fracture stress in parallel (A) and perpendicular direction(B), and anisotropy index of protein phase (C). 
A previous study showed that the fracture stress of aerated material ( $50 \mathrm{rpm}, 5 \mathrm{~min}$ ) is higher than the material without air in the parallel direction. Our explanation was that air incorporation causes the material to become tougher upon deforming with tensile stress. This factor was taken into account by assuming that air bubbles were infinitely deformed (Eq. (3-8)). However, the estimated fracture stress in the parallel direction was still larger than the experimented value of the deaerated materials (Fig. 3-10). This suggests that shearing the aerated dispersion resulted in a stronger protein phase than that in deaerated material. We hypothesis that air bubbles might behave as rigid particles in the protein dispersion during the structuring process, leading to a strengthening of the material. Indeed, non-linear oscillation tests showed that the presence of air bubbles influenced the behaviour of calcium caseinate dispersions. The apparent complex modulus of $30 \mathrm{wt} \%$ aerated dispersion (137 Pa) was higher than that of deaerated dispersion (39 Pa) with an angular frequency of $10 \mathrm{rad} / \mathrm{s}$ and 80\% strain. The apparent complex modulus increased to $143 \mathrm{~Pa}$ if the deaerated dispersion was whipped with a kitchen mixer to again incorporate air. This effect of air is also supported by several studies on other aerated food (Allen et al., 2008; Chin et al., 2005; Tiwari and Bhattacharya, 2011b). For example, dairy cream is converted from a viscous liquid into a viscoelastic solid by whipping (Allen et al., 2008). In a study on aeration of elastic fluids, it was shown that the apparent viscosity of the aerated polyacrylamide-corn syrup dispersion is much higher than for ungassed fluids (Cheng and Carreau, 1994). The presence of air bubbles as a rigid dispersed phase will lead to an increase of the local shear rate in the protein phase. This effect will lead to a more anisotropic protein material.

\subsection{Conclusion}

The mechanical anisotropy of the fibrous materials was described using a load-bearing model. The void fraction, bubble length and width obtained from X-ray tomography was used to calculate the effect of air bubbles on mechanical anisotropy. Those calculations showed that the deformation of air bubbles could not fully account for the anisotropy of calcium caseinate material. The anisotropy of protein phase plays a greater role on the mechanical anisotropy of the fibrous calcium caseinate material. Therefore, we conclude 46 
that the anisotropy of calcium caseinate material is caused by a combination of anisotropy in the protein phase and an additional effect of air.

\section{Acknowledgements}

The authors would like to thank the China Scholarship Council (grant number: 201406820015) for financial support, Friesland Campina for kindly supplying the protein ingredients, Birgit Dekkers for discussions, Remco Hamoen for helping with the X-ray tomography and Jarno Gieteling for technical support. 


\section{Chapter 4}

\section{Thermal history of calcium caseinate affects fibre formation}

This chapter has been submitted as Wang, Z., Dekkers, L. B., \& van der Goot, A. J. (2019). Thermal history of calcium caseinate affects fibre formation. Journal of Food Engineering (under review). 


\begin{abstract}
In this study, we compare the structuring potential and physical properties of spray-dried calcium caseinate (Scaca) and roller-dried calcium caseinate (Rcaca). Scaca formed more pronounced fibrous materials upon shearing compared with Rcaca. The rheological measurements revealed that the Scaca dispersion exhibited more solid-like behaviour. Besides, the particle size in excess water was larger for Rcaca $(2-300 \mu \mathrm{m})$, while Scaca mainly contained small caseinate aggregates $(<800 \mathrm{~nm})$. Finally, Rcaca was less susceptible to enzymatic crosslinking with transglutaminase. We explain differences in structuring potential and physical properties by the intensive thermal treatment during roller drying for Rcaca. The application of a similar thermal process to Scaca resulted in similar properties as Rcaca. We conclude that the process history of calcium caseinate has major consequences for its fibre formation potential.
\end{abstract}




\subsection{Introduction}

Casein, a composite of four different proteins ( $\alpha_{\mathrm{S1} 1^{-}}, \alpha_{\mathrm{S} 2^{-}}, \beta-$, and $\kappa^{-}$-casein), are held together by colloidal calcium phosphate, which can be removed by precipitation of casein at $\mathrm{pH} 4.6$ and washing. The acid casein can be resuspended by increasing the pH with $\mathrm{NaOH}$ or $\mathrm{Ca}(\mathrm{OH})$ 2. The resulting sodium and calcium caseinate slurries may then be spraydried or roller-dried for further applications (Dalgleigh and Law, 1988; Fox and Mulvihill, 1982; Huppertz et al., 2017). The solid content of the slurry is $\sim 20 \mathrm{wt} \%$ prior to spray drying and $\sim 40$ wt\% prior to roller drying (O'Regan and Mulvihill, 2011). Heating is necessary to reduce viscosity during neutralization and prior to drying (Augustin et al., 2011). The time that caseinate slurries are kept at high temperature during pre-treatment and drying is minimized because browning may occur otherwise, amongst others due to reactions between the proteins and residual lactose (Mulvihill and Ennis, 2003).

Generally, thermally induced changes mostly occur during roller drying and only sporadic with spray drying (Schuck, 2011). Spray-dried caseinate powder has excellent solubility, flavour and colour. Roller drying results in powder that is more scorched and therefore has a less desirable flavour profile than spray-dried powder. This is the reason that roller drying is therefore not widely used for dairy powder (Chandan and Kilara, 2011). However, roller-dried caseinate powder is cheaper, has better dispersibility in water and yields more viscous solutions than spray-dried caseinate at similar concentrations (Carr and Golding, 2016; Carr et al., 2003; Fox and Mulvihill, 1982). Roller-dried caseinates are mostly used in meat products and processed cheese. Commercial roller-dried sodium caseinates are less sensitive to $\mathrm{Ca}^{2+}$ compared with spray-dried caseinates (Dalgleigh and Law, 1988).

Our previous research showed that $30 \mathrm{wt} \%$ calcium caseinate dispersions can form fibrous structures using a technology based on simple shear deformation (Manski et al., 2007a). The presence of $\mathrm{Ca}^{2+}$ is important for structure formation because this structure was not formed with a $30 \mathrm{wt} \%$ sodium caseinate dispersion using the same processing conditions (Manski et al., 2007c). Concurrent enzymatic cross-linking by 
transglutaminase and incorporation of air can enhance the anisotropy of calcium caseinate fibres (Wang et al., 2019a). All those studies were done using spray-dried calcium caseinate. We observed, though, that different batches of spray-dried calcium caseinate powder possessed different properties and the final products could have a different degree of anisotropy, despite the use of similar concentrations and process conditions (Grabowska et al., 2012; Manski et al., 2007a; Wang et al., 2019a). Differences in structures obtained became even more explicit when comparing spray-dried and roller-dried calcium caseinate (Tian et al., 2018; Wang et al., 2019a). Therefore, we hypothesize that the drying process of powder could influence their structuring potential.

This study focusses on understanding differences in spray-dried and roller-dried calcium caseinate powder through investigating their physical properties and structuring potential. The sheared materials were prepared in a shear cell device using deaerated dispersions and aerated dispersions with or without transglutaminase. The resulting products were investigated with tensile tests, X-ray tomography (XRT), and scanning electron microscopy (SEM).

\subsection{Materials and methods}

\subsubsection{Materials}

Spray-dried (Scaca) and roller-dried (Rcaca) calcium caseinate powders were produced by DMV International (Veghel, the Netherlands) from the same milk source. Scaca powder was prepared from a slurry of $\sim 22 \mathrm{wt} \%$ solid. Spray drying was conducted using a feed temperature $82 \pm 2^{\circ} \mathrm{C}$, an air inlet temperature $230-240^{\circ} \mathrm{C}$ and an air outlet temperature $78^{\circ} \mathrm{C}$. The drying air-flow rate was $90,000 \mathrm{~kg}$ dry air/h. The nozzle (Delevan) pressure was 157 bar. Rcaca powder was prepared from a slurry containing 45 wt\% solid. The feed temperature was $120-130^{\circ} \mathrm{C}$. The hot drum temperature was $100^{\circ} \mathrm{C}$. The layer thickness on the hot drum was $1 \mathrm{~mm}$.

The resulting Scaca and Rcaca powders had a dry matter content of $94.5 \mathrm{wt} \%$ and 93.9 wt $\%$, respectively. The powder size distribution was determined using a Mastersizer 
3000 (Malvern Instruments, Malvern, UK). The powder bulk density was measured with a 100-mL cylinder. The calcium content in the powders was determined using an inductively coupled plasma mass spectrometer. Colour measurement of the powders was carried out using a chromameter (CR-400, Konica Minolta, Japan) and a data processor (DP-400, Konica Minolta, Japan).

The activity of microbial $\mathrm{Ca}^{2+}$-independent transglutaminase derived from Streptoverticillium moberansae (1 wt\% transglutaminase, 99 wt\% maltodextrin; Ajinomoto, Tokyo, Japan) was 114 units $\cdot g^{-1}$ determined by the hydroxamate method (Yokoyama et al., 2003). The term "enzyme" in this study refers to the transglutaminase product. A $20 \mathrm{wt} \%$ transglutaminase solution was stirred at room temperature for $1 \mathrm{~h}$. Transglutaminase was used at an enzyme/protein ratio of 1:20 based on weight.

\subsubsection{Particle size measurement}

Scaca and Rcaca powder were mixed into deionized water to obtain a $10 \mathrm{wt} \%$ protein dispersions overnight. The particle size distribution was determined using a Mastersizer 3000.

\subsubsection{Confocal scanning laser microscopy}

Rhodamine B was added to the dispersions. Dispersions were visualized with an CSLM 510 microscope (Zeiss, Oberkochen, Germany) using an Ar-ion laser (543 nm) with an emission filter of 561-711 $\mathrm{nm}$ and a $63 \times$ oil-immersion objective (NA 1.4).

\subsubsection{Rheological properties}

Strain sweep measurements within a strain range of $0.1 \%-100 \%$ were performed to determine the linear viscoelastic region of $30 \mathrm{wt} \%$ calcium caseinate dispersions at $50^{\circ} \mathrm{C}$ using a Paar MCR 301 rheometer (Anton Paar, Graz, Austria). Frequency sweeps (angular frequency $\omega$ range $0.1-100 \mathrm{rad} / \mathrm{s}$ ) were performed at a constant strain of $1 \%$ at $50^{\circ} \mathrm{C}$. Serrated parallel-plates geometry (diameter $25 \mathrm{~mm}$, gap $1 \mathrm{~mm}$ ) was used. A solvent trap was used to prevent water evaporation. For these experiments, 30 wt $\%$ protein dispersions were mixed at $80^{\circ} \mathrm{C}$ for 10 min to make them fully homogeneous. 


\subsubsection{Material preparation in the shear cell device}

A protein dispersion was prepared by manually mixing $30 \mathrm{wt} \%$ calcium caseinate powder and water(with or without transglutaminase solution) for 1 min using a spatula. The dispersion was placed in a shearing device consisting of a rotating and a stationary cone, which were both thermostatted with a heating and a cooling water bath. We used the same shear cell device and methods for material preparation as described previously (Wang et al., 2019a). A schematic drawing of the shear cell geometry appears in an earlier publication (van der Zalm et al., 2012). The dispersion was transferred to the pre-heated $\left(50^{\circ} \mathrm{C}\right)$ shearing device and subsequently processed at the same temperature with a rotating speed of $50 \mathrm{rpm}$ for $5 \mathrm{~min}$. After processing, the samples were cooled to $4^{\circ} \mathrm{C}$ in 10 min before they were removed from the device. Tensile tests were performed on materials within $1 \mathrm{~h}$ after discharge from the device. Parts of the materials were stored at $-20^{\circ} \mathrm{C}$ until further analysis.

To obtain material without air bubbles, deaeration was performed on protein dispersions without transglutaminase by heating $\left(80^{\circ} \mathrm{C}, 5 \mathrm{~min}\right)$, centrifuging and application of a long rest time before shearing (Wang et al., 2019a). An extra experiment was done to elucidate how additional heat treatment influences the structuring potential. Before material preparation, $30 \mathrm{wt} \%$ Scaca dispersion was heated at $130^{\circ} \mathrm{C}$ for $3 \mathrm{~min}$ and cooled down to room temperature in $10 \mathrm{~min}$. The particle size distribution of the resulting dispersion was also measured. To elucidate the effect of $\mathrm{Ca}^{2+}$ on the structure formation, extra $\mathrm{CaCl}_{2}$ was dissolved in a water phase before preparing an Rcaca dispersion with and without air bubbles.

\subsubsection{Tensile strength analysis}

A texture analyser (Instron Testing System, table model 5564) was used with a load cell of $100 \mathrm{~N}$. A dog-bone-shaped mould was used to cut samples for tensile tests. The samples between the two clamps were $15.2 \mathrm{~mm}$ long and $3.18 \mathrm{~mm}$ wide; the thickness varied between 4 and $6 \mathrm{~mm}$. The samples were taken parallel and perpendicular to the shear flow (based on the shear-vorticity plane). The tensile tests were conducted at a constant 
deformation speed of $3 \mathrm{~mm} \cdot \mathrm{s}^{-1}$. Grips with abrasive paper were used to prevent slipping during testing. At least three specimens per direction were measured. The results from these tensile tests were depicted as force-displacement curves. The point following a dramatic decrease in stress in the stress-strain curve was taken as the fracture point. This point determined fracture stress $(\sigma, \mathrm{kPa})$ and fracture strain $(\varepsilon,-)$. Young's Modulus $(E$, $\mathrm{kPa}$ ) was calculated by applying a linear fit $(E=\sigma / \varepsilon)$ using the initial displacement, which was taken as the interval between 0 and $4.5 \mathrm{~mm}$ displacement. The apparent strain hardening coefficient $n$ was calculated by applying a power law fit $\left(\sigma=k \cdot \varepsilon^{n}\right)$ in the strain interval between $30 \%$ and $100 \%$ of fracture strain $\varepsilon$. When $n$ exceeds 1 , the sample exhibits strain hardening. $k$ is the strength coefficient, a mechanical property that indicates the stiffness of the material. The ratio of the average values measured in the parallel and perpendicular directions to the shear flow was used as an indication of the mechanical anisotropy of the samples.

\subsubsection{X-ray tomography}

Samples were scanned with a GE Phoenix v|tome|x m tomographer (General Electric, Wunstorf, Germany) set at $80 \mathrm{kV} / 90 \mu \mathrm{A}$. The system contains two X-ray sources. The 240 $\mathrm{kV}$ micro focus tube with tungsten target was used. The images were recorded by a GE DXR detector array with $2024 \times 2024$ pixels (pixel size $200 \mu \mathrm{m}$ ). The detector and object were located $815 \mathrm{~mm}$ and $28.55 \mathrm{~mm}$ from the X-ray source, respectively, resulting in a spatial resolution of $7.00 \mu \mathrm{m}$. A full scan consisted of 750 projections over $360^{\circ}$. The first image was skipped; images 2-4 were averaged into one projection. GE reconstruction software (Wunstorf, Germany) was used to calculate the 3D structure via back projection. The 3D images were analysed using Avizo imaging software version 9.3.0, including the total bubble volume, total gas volume, gas void fraction and every single bubble volume. The degree of deformation (Deff) was defined as:

$$
\operatorname{Def}=(a-b) /(a+b)
$$

where $a$ is the length of a deformed air bubble parallel to the shear flow, and $b$ is the average width of the air bubble perpendicular to the shear flow (based on velocity 
gradient-shear flow plane and vorticity-shear flow plane). Def $=0$ represents a spherical air bubble; Def $=1$ represents an infinitely deformed air bubble.

\subsubsection{Scanning electron microscopy}

The sheared calcium caseinate materials were torn apart by hand along the shear flow direction $(\sim 2 \mathrm{~mm}$, based on the shear flow-vorticity plane), and rinsed with demineralized water. Subsequently, the samples were fixed in a glutaraldehyde solution $(2.5 \% \mathrm{v} / \mathrm{v})$ for $1 \mathrm{~h}$ and dehydrated in a graded series of ethanol $(10 \%, 30 \%, 50 \%, 70 \%$, 90\% and 100\% v/v, 20 min each). Critical point drying with carbon dioxide (CPD 020, Balzers, Liechtenstein) was performed. The dried samples and powder were then glued onto sample holders using conductive carbon cement (Leit-C, Neubauer Chemicalien, Germany) and carbon tabs (SPI Supplies/Structure Probe, West Chester, USA) respectively, and subsequently sputter coated with $15 \mathrm{~nm}$ platinum (JFC 1200, JEOL, Japan). The surface was observed with a field emission scanning electron microscope (Magellan 400, FEI, Eindhoven, the Netherlands) at ambient temperature using a working distance of 4-8 mm, with secondary electron detection at $2 \mathrm{kV}$ and $6.3 \mathrm{pA}$.

\subsection{Results}

We compared the physical and rheological properties of dispersions of spray-dried (Scaca) and roller-dried calcium caseinate powders (Rcaca), and their ability to form fibrous structures.

\subsubsection{Characterization of calcium caseinate powders}

Fig. 4-1A shows the particle size distribution of spray-dried and roller-dried powder. The peak particle size distribution was similar for the two powders, although the distribution of roller-dried powder was broader than the distribution of spray-dried powders. The spray-dried powder particles were spherical and porous (Fig. 4-1B), whereas the rollerdried powder particles were irregular and angular in shape (Fig. 4-1C). The Scaca powder had lower bulk density $\left(392.7 \mathrm{~g} \cdot \mathrm{L}^{-1}\right)$ than Rcaca $\left(563.4 \mathrm{~g} \cdot \mathrm{L}^{-1}\right)$, partly due to the occluded air in spray-dried powder particles. The colour was analysed in terms of Chroma values 
$\left(C^{*}\right)$, which is a measure for the intensity of visual colour. Rcaca powder showed higher $C^{*}(11.2)$ than Scaca powder (5.8). The higher value of Rcaca powder indicated a less white powder, possibly suggesting colouring due to reaction between calcium caseinate and residual lactose (Morales and Van Boekel, 1998). Scaca and Rcaca powder contained $1.52 \mathrm{wt} \%$ and $1.33 \mathrm{wt} \%$ calcium, respectively.

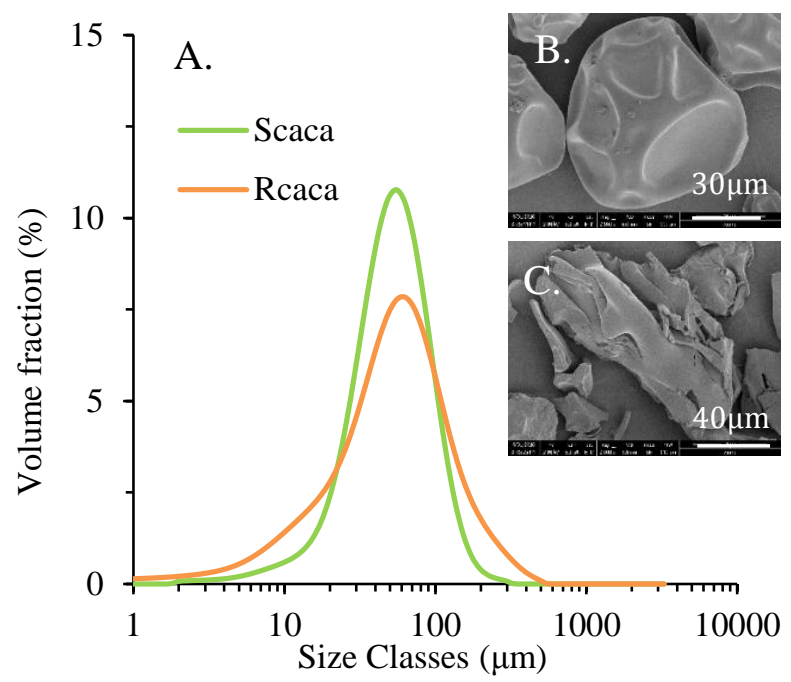

Fig. 4-1. Size distribution of powders (A); SEM images of spray-dried powder (B) and roller-dried powder (C).

We measured the particle size distribution of $10 \mathrm{wt} \%$ dispersions after overnight mixing using a Mastersizer (Fig. 4-2), which gives a quick assessment of difference particle size distributions in Scaca and Rcaca dispersions. The Scaca dispersion had a bimodal particle size distribution: peak 1 contained $63.2 \%$ of small particles by volume, representing caseinate aggregates smaller than $800 \mathrm{~nm}$. Peak 2 contained large particles with sizes ranging from 2-100 $\mu \mathrm{m}$. The Rcaca dispersion showed a unimodal particle size distribution in the range of $2-300 \mu \mathrm{m}$. The dispersion was centrifuged $(18,400 \mathrm{~g}$ and 5 min) to check for smaller particles in the Rcaca dispersion. The particles in the supernatant had a bimodal particle size distribution. Therefore, it can be concluded that both Scaca and Rcaca had both particles on a nano- and micro-length scale, but Rcaca dispersion contained more larger particles and fewer small aggregates than Scaca dispersion, which was also confirmed by CSLM images (Fig. 4-2B, C). 
Heat-induced aggregation during powder processing could explain the different size distribution between Scaca and Rcaca dispersion. To test this hypothesis, we heated a Scaca dispersion. After heating at $130^{\circ} \mathrm{C}$ for $3 \mathrm{~min}$, this Scaca dispersion had a similar particle size distribution as the Rcaca dispersion. The results here were in line with previous studies using milk powder that showed that the differences in micelle size were a result of differences in the drying process (spray and roller drying) (Kalab and Emmons, 1974). The casein micelles in the spray-dried milk powders retained a globular shape (diameter $\sim 100 \mathrm{~nm}$ ), whereas larger casein micelles (clusters) were present in rollerdried milk powder. Heat-induced aggregation was also reported for casein and sodium caseinate dispersions (HadjSadok et al., 2008; Loveday et al., 2010; Ono et al., 1999; Quo et al., 1989).
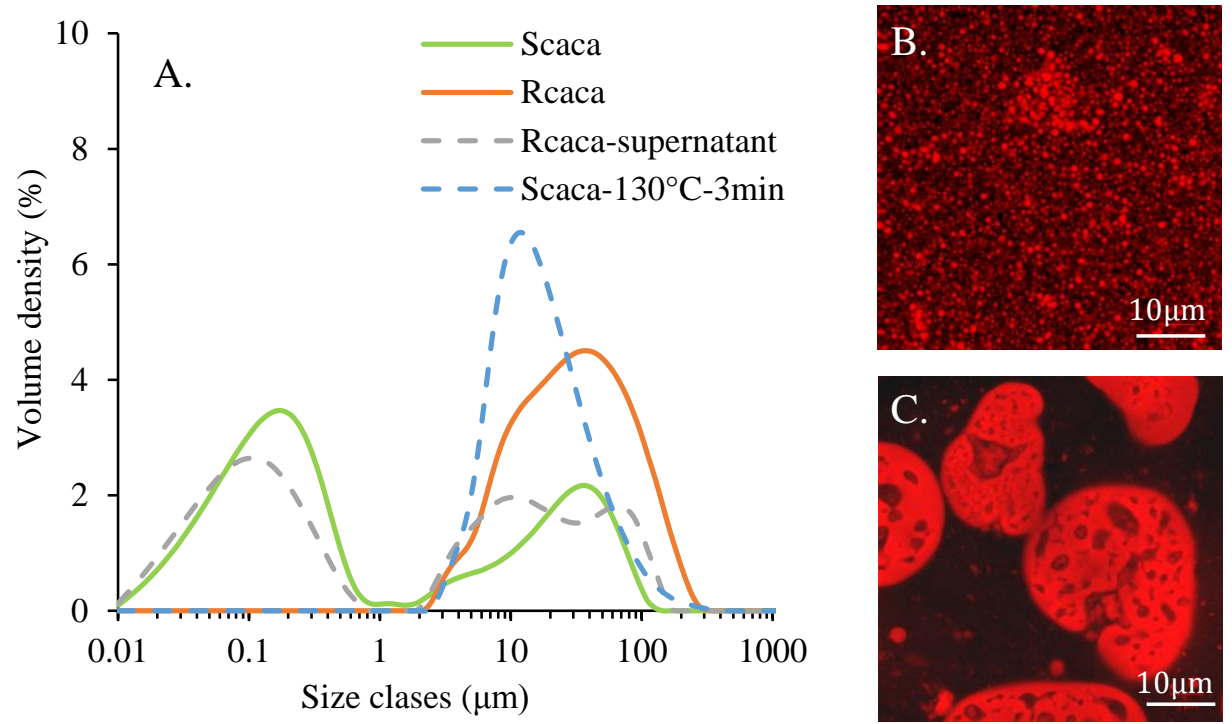

Fig. 4-2. A: Particle size distribution of $10 \mathrm{wt} \%$ Scaca and Rcaca dispersions after overnight mixing. The grey dashed line represents the supernatant of Rcaca dispersion after centrifugation $(18,400 \mathrm{~g}$ and $5 \mathrm{~min})$. The blue dashed line represents a Scaca dispersion after heating at $130^{\circ} \mathrm{C}$ for $3 \mathrm{~min}$. CLSM images of Scaca (B) and Rcaca dispersion (C) after overnight mixing.

\subsubsection{Rheological properties of calcium caseinate}

Fig. 4-3 shows the viscoelastic characteristics of $30 \mathrm{wt} \%$ Scaca and Rcaca dispersions $\left(50^{\circ} \mathrm{C}\right)$. The inverse of the angular frequency at the crossover of storage modulus $\left(G^{\prime}\right)$ and 
loss modulus $\left(G^{\prime \prime}\right)$ is defined as the relaxation time $(\tau)$. Both $G^{\prime}$ and $G^{\prime \prime}$ of the Rcaca dispersion were higher than those of the Scaca dispersion. However, the $\tau$ value of Rcaca was lower than that of Scaca, indicating less solid-like behaviour for Rcaca dispersion. In calcium caseinate dispersion, $\mathrm{Ca}^{2+}$ provides strong interactions between proteins, largely influencing its rheological behaviour (Thomar et al., 2014, 2013). Addition of $\mathrm{CaCl}_{2}$ to dense sodium caseinate led to a reduction of $G^{\prime}$ and an increase in $\tau$ (Thomar et al., 2012). We added $\mathrm{CaCl}_{2}$ to dense Rcaca dispersion to have the same $\mathrm{Ca}^{2+}$ content as in the Scaca dispersion, which resulted in an increase of $G^{\prime}$ and little change in the $\tau$ value. This rheological behaviour remained different with the Scaca dispersion (i.e. $\tau$ value was still lower in case of Rcaca with extra $\mathrm{Ca}^{2+}$ ), suggesting that Rcaca was less sensitive to $\mathrm{Ca}^{2+}$. Similar observations were described for sodium calcium caseinate dispersions to which $\mathrm{Ca}^{2+}$ was added (Dalgleigh and Law, 1988).

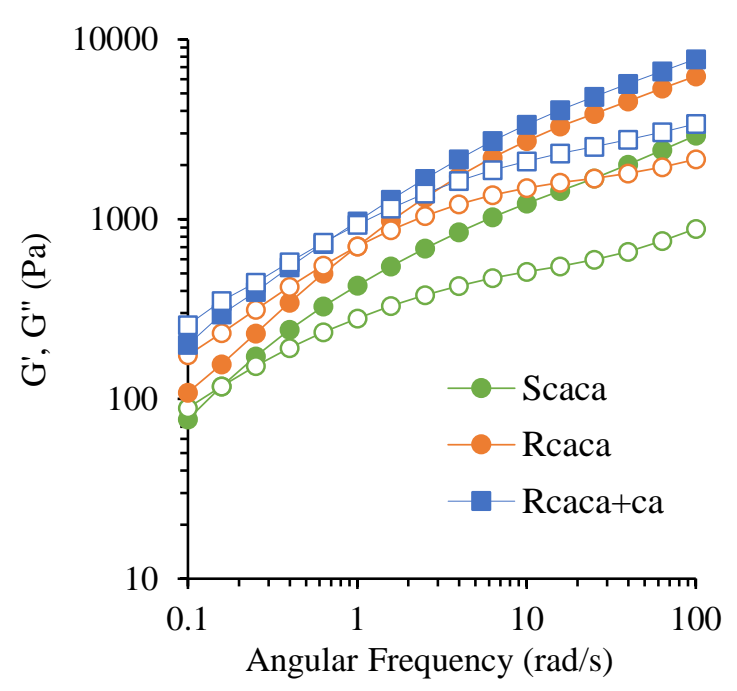

Fig. 4-3. Storage modulus $G$ (filled), loss modulus $G^{\prime \prime}$ (open) as a function of angular frequency of $30 \mathrm{wt} \%$ Scaca and Rcaca dispersion measured at $50^{\circ} \mathrm{C}$.

\subsubsection{Shear-induced structuring}

Dense calcium caseinate dispersions can be transformed into anisotropic structures through shear deformation. Previous studies showed that concurrent enzymatic crosslinking and air incorporation can enhance the anisotropy of calcium caseinate fibres (Wang et al., 2019a). In this study, we compared the structures of Scaca and Rcaca and investigated the effects of air inclusion and transglutaminase. 


\subsubsection{Macrostructure}

The application of shear $\left(50 \mathrm{rpm}, 5 \mathrm{~min}, 50^{\circ} \mathrm{C}\right)$ to calcium caseinate dispersions yielded materials with different macrostructures. Fig. 4-4 shows that the sheared and crosslinked Scaca material had a more pronounced fibrous appearance than Rcaca material. The latter material showed a layered structure. When tearing the Scaca material along the direction of the shear flow, thin and long fibres appeared (Fig. 4-4A). Those clearly separated fibres were hardly present in Rcaca material ( Fig. 4-4B).
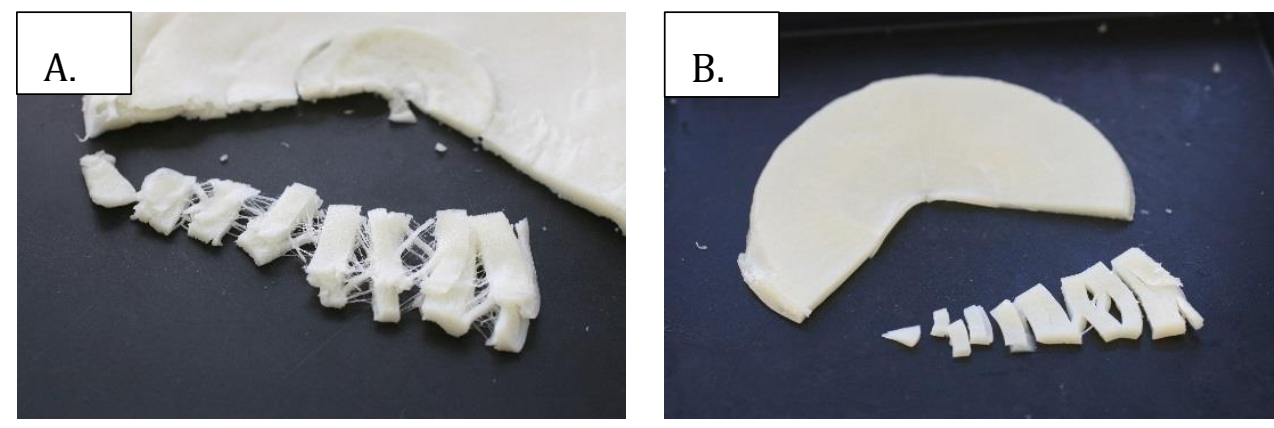

Fig. 4-4. Sheared calcium caseinate dispersion at $50 \mathrm{rpm}$ for $5 \mathrm{~min}$. A: Scaca with transglutaminase; B: Rcaca with transglutaminase.

\subsubsection{Meso- and microstructure}

Meso- and microstructures of Scaca and Rcaca materials were observed with SEM (Fig. 4-5). The sheared and cross-linked Scaca material was torn apart by hand along the shear flow direction. Its fracture surface had long and clear lines (Fig. 4-5A). The orientation of the lines close to air bubbles followed the curvature of the bubbles, which is in line with our previous observations (Wang et al., 2019a). In the Rcaca material, the lines were less explicit (Fig. 45B). Further magnification $(50,000 \times)$ revealed that the Scaca material contained clear alignment parallel to the shear flow direction even at small length scale; this alignment was less visible in the Rcaca material (Fig. 4-5C, D). Clearly, both materials showed anisotropy at a mesoscale; while only Scaca material had orientation at a smaller length scale. 


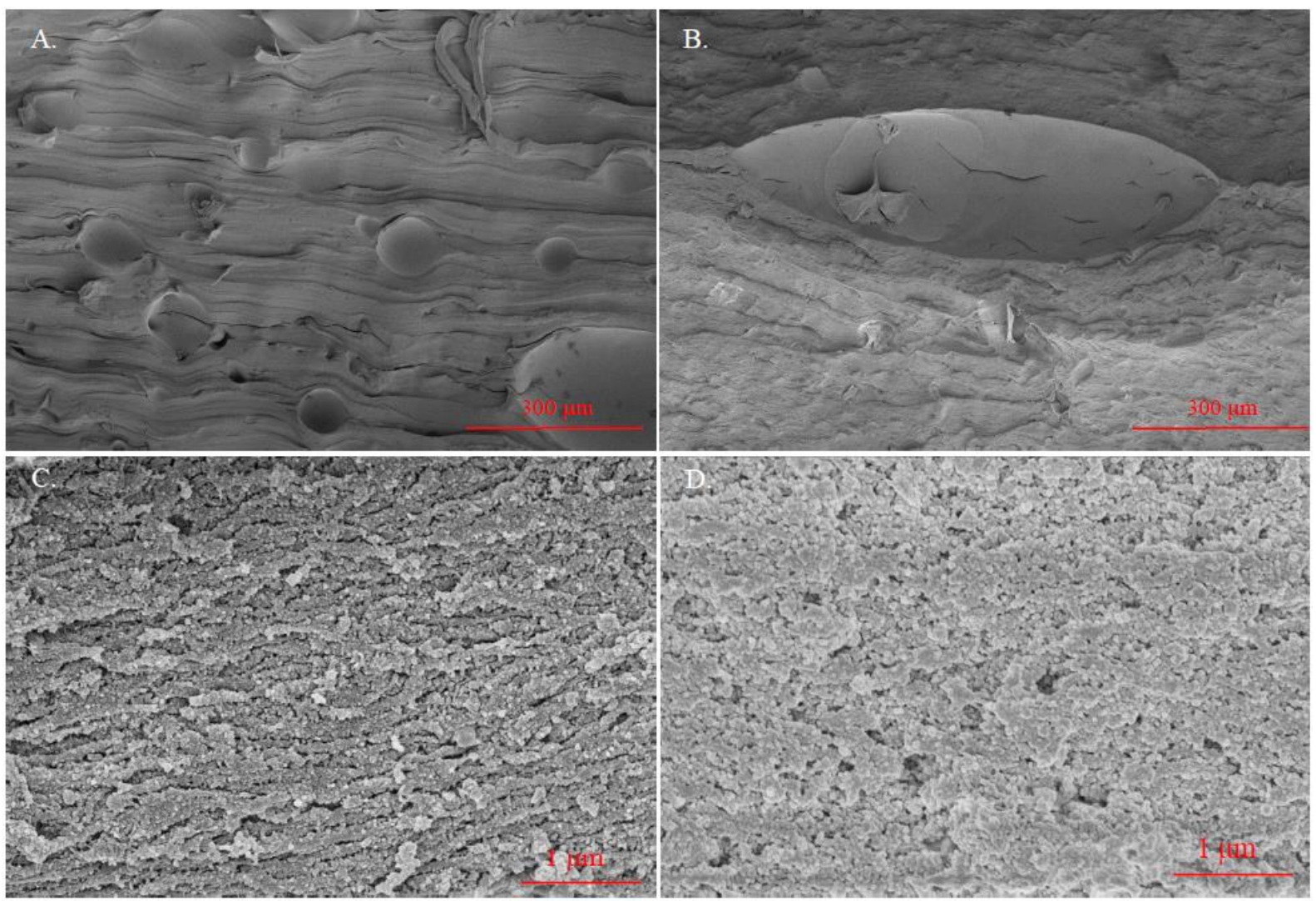

Fig. 4-5. SEM images of sheared and cross-linked Scaca (A, C) and Rcaca material (B, D). The images were taken from the flow-velocity gradient plane.

\subsubsection{Air bubbles}

The void fractions of air in calcium caseinate materials were measured with XRT (Fig. 46A). Non-sheared materials were also prepared in the shear cell device $(0 \mathrm{rpm}, 5 \mathrm{~min}$, $\left.50^{\circ} \mathrm{C}\right)$. Without shearing, more air was present in Scaca material $(20.3 \pm 0.4 \%, \mathrm{v} / \mathrm{v})$ than in Rcaca material $(8.2 \pm 0.6 \%, v / v)$, which is probably a result of the fact that spray-dried powder had more occluded air inside the initial powder particles. After shearing, the void fractions of air in Scaca decreased to $15.9 \pm 0.1 \%(\mathrm{v} / \mathrm{v})$ and $5.1 \pm 0.6 \%(\mathrm{v} / \mathrm{v})$, respectively. When processed with the addition of transglutaminase, the void fraction in Scaca material was higher than that of material processed without transglutaminase. The void fraction in Rcaca material was hardly influenced by enzyme addition. Overall, Scaca materials contained more air bubbles compared with Rcaca materials. 
XRT analysis showed that air bubbles in sheared and cross-linked Scaca and Rcaca materials were deformed differently (Fig. 4-6B). In Scaca materials, the small bubbles $\left(<10^{-3} \mathrm{~mm}^{3}\right)$ were not deformed $(\sim 0)$ and the large air bubbles $\left(>10^{-3} \mathrm{~mm}^{3}\right)$ were only slightly deformed (0.1-0.25). In Rcaca materials, the air bubbles in the largest category $\left(>10^{-1} \mathrm{~mm}^{3}\right)$ were deformed $(\sim 0.23)$, while air bubbles $\left(<10^{-1} \mathrm{~mm}^{3}\right)$ were not deformed.
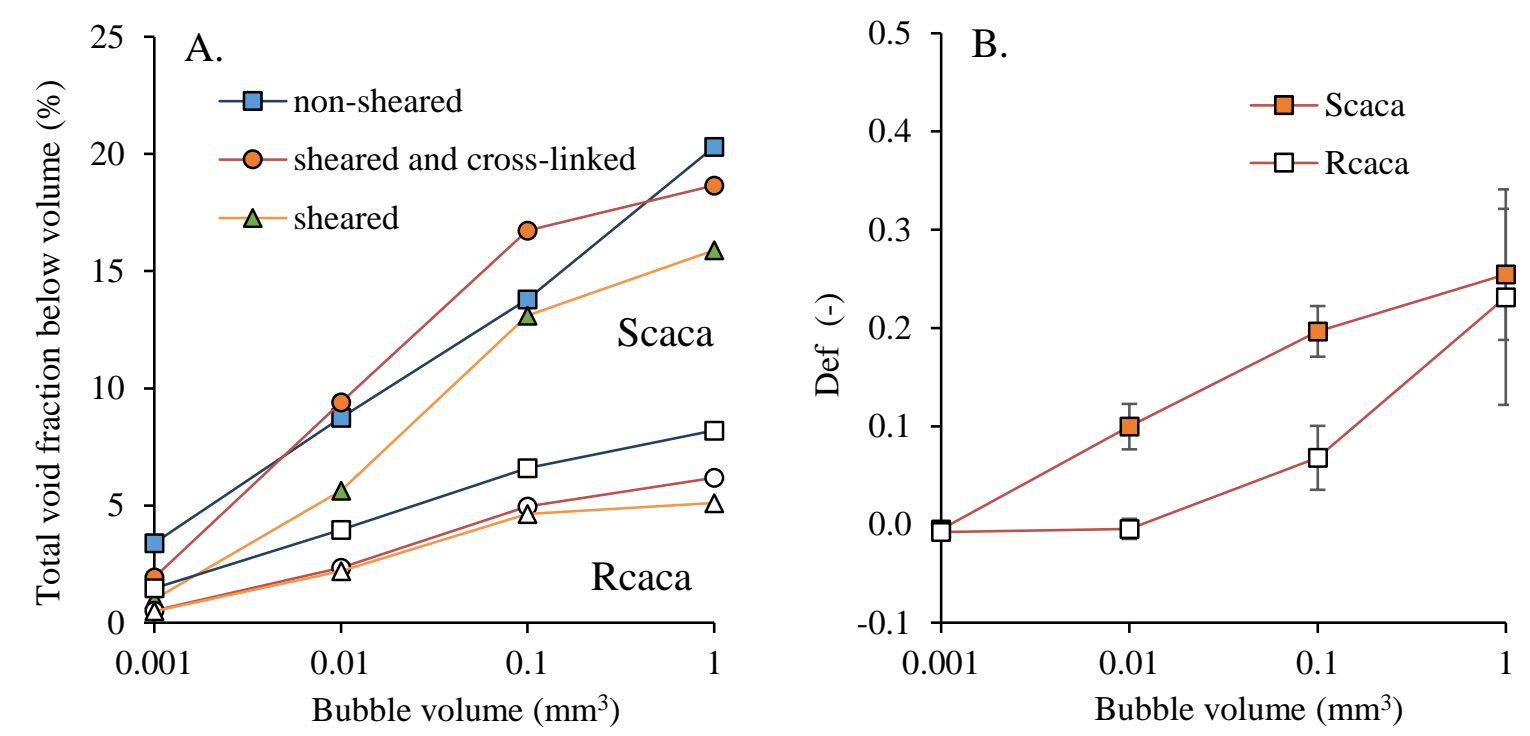

Fig. 4-6. A: Overall cumulative void fraction of air in calcium caseinate materials (as the void fraction below the bubble volume in $\mathrm{mm}^{3}$ ). B: Associated deformation of the bubbles in sheared and cross-linked Scaca and Rcaca materials.

\section{3-3.4. Tensile properties}

Fig. 4-7 shows fracture stress $\sigma$, fracture strain $\varepsilon$, Young's modulus $E$ and strain hardening coefficient $n$ and the corresponding anisotropy indices for Scaca and Rcaca materials. Anisotropy indices of Scaca materials on fracture properties were larger than those of Rcaca materials, which corresponded to a more fibrous appearance of Scaca materials (Fig. 4-4). The results confirmed that Scaca powder has better potential for creating fibrous structures. Sheared materials were also prepared in the absence of air and transglutaminase to better determine the differences in the protein properties of both caseinates. The Scaca material had a higher $\sigma$ and $\varepsilon$ in the parallel direction $(80 \mathrm{kPa}, 0.96)$ than Rcaca material ( $42 \mathrm{kPa}, 0.73)$. In the perpendicular direction, the fracture properties were similar for both Scaca and Rcaca material. Scaca material showed anisotropy in $E$ 
and $n$, whereas Rcaca material showed hardly any anisotropy in these properties. These differences clearly suggest that the protein in Scaca and Rcaca behaved differently upon shear deformation.

With air bubbles incorporated in Scaca materials, the anisotropy index of $\sigma$ increased from 3.9 to 6.6 (Fig. 4-7A). However, this increase in anisotropy index upon air inclusion was not found in Rcaca materials. Similar observations were found for $\varepsilon$ and $n$ (Fig. 4-7B, D). The air incorporation in Scaca enhanced mechanical anisotropy, but this was not the case for Rcaca material. Furthermore, the presence of transglutaminase largely increased all mechanical properties of Scaca in the parallel direction, whereas such reinforcement of transglutaminase was much less when Rcaca material was used. A possible explanation is that thermal-induced damage due to oxidation during roller drying resulted in fewer $\varepsilon$ $\mathrm{NH}_{2}$ groups of lysine available for cross-linking with transglutaminase (Rolls and Porter, 1973). 

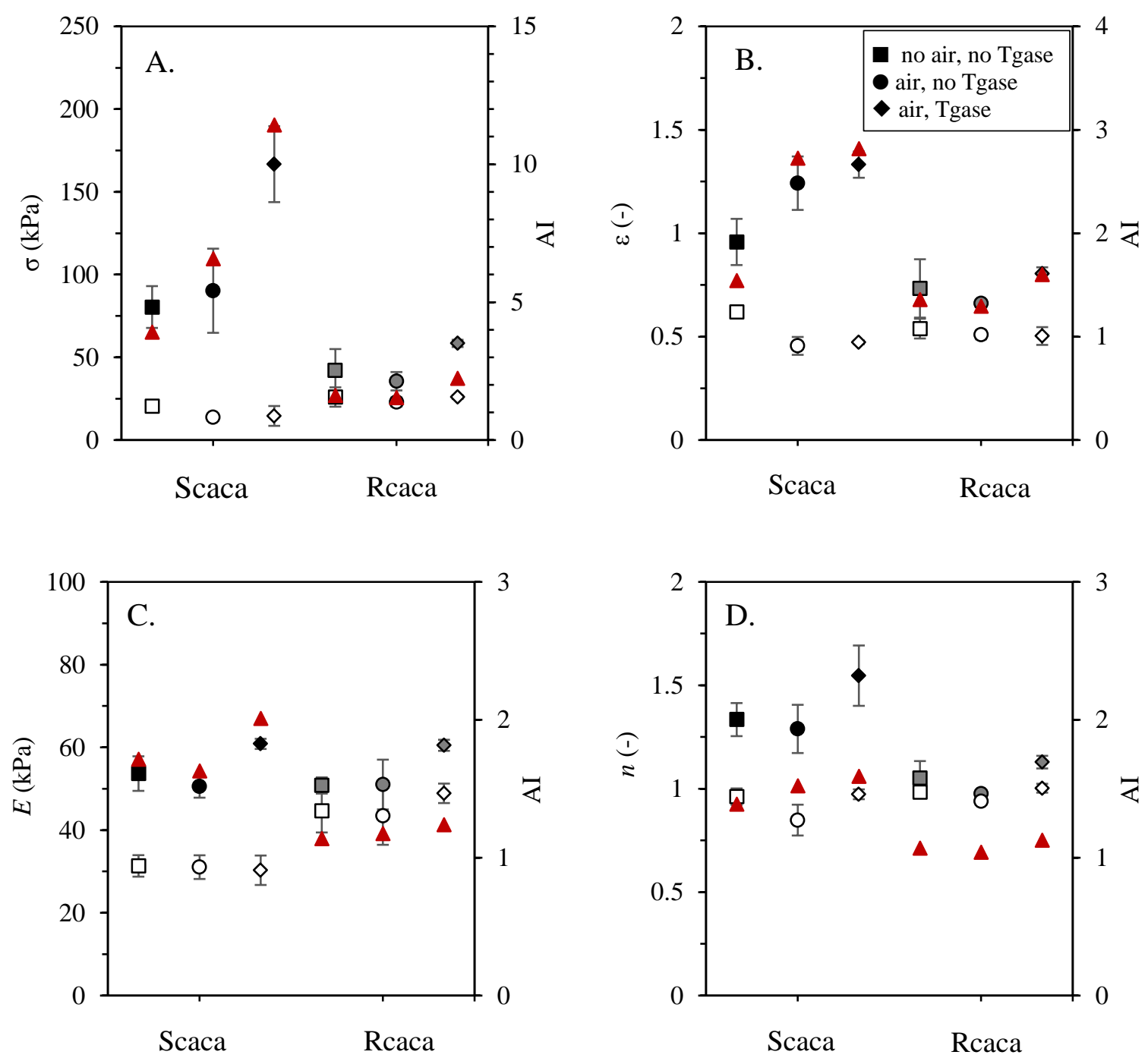

Fig. 4-7. Tensile properties: (A) fracture stress $\sigma$, (B) fracture strain $\varepsilon$, (C) Young's modulus Eand (D) strain hardening coefficient $n$ of $30 \mathrm{wt} \%$ Scaca (black) and Rcaca (grey) materials. The error bars for the tensile properties are \pm standard deviation. The anisotropy index $\mathrm{AI}(\boldsymbol{\Delta})$ is the ratio between the average values measured in the parallel (closed bar) and perpendicular (open bar) directions to the shear flow.

\subsection{Discussion}

We found differences in $\mathrm{Ca}^{2+}$ content in Scaca and Rcaca, which could influence fibrous structure formation. Previous research showed that addition of a calcium chelator, sodium triphosphate pentabasic, prevented formation of an anisotropic structure of calcium caseinate (Grabowska et al., 2012). Thomar et al. (2013) stated that the behaviour of calcium caseinate is the same as that of sodium caseinate with the addition 
of $\mathrm{CaCl}_{2}$. Following this, $\mathrm{CaCl}_{2}$ was added to a Rcaca dispersion to exclude the effect of differences in $\mathrm{Ca}^{2+}$ content on rheological properties and structure formation. It was observed that the rheological properties changed slightly when $\mathrm{Ca}^{2+}$ was added to Rcaca to reach a similar concentration as in Scaca, but the rheological properties were still different compared with the Scaca dispersion (Fig. 4-4). It was found though that shearing of Rcaca dispersion with extra $\mathrm{CaCl}_{2}$ resulted in a fibrous material, containing more air $(8.6 \%, v / v)$ than the original Rcaca material $(5.1 \%, v / v)$. Addition of $\mathrm{CaCl}_{2}$ slightly increased anisotropic indices of fracture properties. No significant differences were found for Young's modulus and strain hardening. Nevertheless, the Rcaca dispersion with extra $\mathrm{CaCl}_{2}$ still had different properties compared with Scaca dispersion. Here, we suspect the cause to be related to heat-induced changes on the properties of Rcaca. Those changes include a reduced sensitivity to $\mathrm{Ca}^{2+}$ and oxidation of lysine groups (Dalgleigh and Law, 1988; Quo et al., 1989).

Moughal et. al also reported that commercial calcium caseinate has a bimodal particle size distribution, although they did not correlate this to a thermal history (Moughal et al., 2000). Here, both the Scaca and Rcaca dispersions had a bimodal nature of particle size distribution (Fig. 4-2). However, caseinate aggregates $(<800 \mathrm{~nm})$ were present at a much higher concentration in Scaca dispersion, while large particles $(2-300 \mu \mathrm{m})$ were mainly found in Rcaca dispersion. This could be explained by the severe thermal treatment during roller drying. Shearing Rcaca dispersion with large structural elements (particles) did not show any anisotropy in Young's modulus and strain hardening. Therefore, we concluded that alignment did not occur at small length scale. Differences in mechanical properties were caused by elongated air bubbles between the large particles present in Rcaca. It seems that protein aggregates as largely present in Scaca can cause orientation at smaller length scale upon shearing.

The lower susceptibility of Rcaca material to transglutaminase addition could also be related to its severe thermal history. The effect of heating was further demonstrated by shearing a Scaca dispersion that was heated $\left(130^{\circ} \mathrm{C}, 3 \mathrm{~min}\right)$. That Scaca did not give any fibres. After heating, the Scaca gave a similar size distribution as Rcaca dispersion (Fig. 4- 
2) and had lost air present in the dispersions. As a result, no fibre formation was observed anymore. (appendix 4A-1).

Air inclusion plays an important role for fibrous appearance and mechanical anisotropy in Scaca fibre formation (Wang et al., 2019b, 2019a). In case of Scaca, it was suggested that those air bubbles behaved like rigid particles due to the strain hardening of the Scaca protein that surrounded the air bubbles. Thus, small and non-deformed air bubbles are observed in Scaca material. However, in case of Rcaca material, mechanical anisotropy was a result of the presence of a few large and deformed air bubbles. We therefore conclude that the formation of anisotropic materials is based on a different mechanism. The anisotropy of Scaca is based on alignment of small aggregates that give rise to strain hardening properties. In case of Rcaca, it seems that the anisotropy is caused by large and deformed air bubbles. The latter mechanism seems is line with structure formation reported for plant-based materials, such as a blend of soy protein isolate-pectin material as described in a previous study (Dekkers et al., 2018).

\subsection{Conclusion}

This study shows the large differences in structuring potential between spray-dried and roller-dried calcium caseinate. Shearing dense Scaca dispersions resulted in fibrous materials, whereas shearing dense Rcaca dispersions yielded layered materials with less mechanical anisotropy. These differences were ascribed to the more intensive thermal treatment during roller drying. Heat-induced changes in Rcaca dispersion reduced the susceptibility to enzymatic crosslinking through transglutaminase, as well as its susceptibility to $\mathrm{Ca}^{2+}$. The latter led to a less solid-like behaviour. Clearly, it is necessary to pay attention to the thermal history of ingredients for fibre formation.

\section{Acknowledgements}

The authors would like to thank the China Scholarship Council (grant number 201406820015) for financial support, Friesland Campina for kindly supplying the protein 
ingredients and drying parameters, Hedde Paulusma and Eric van de Ven for fruitful discussions, and Jarno Gieteling for technical support. 


\section{Appendix 4A}

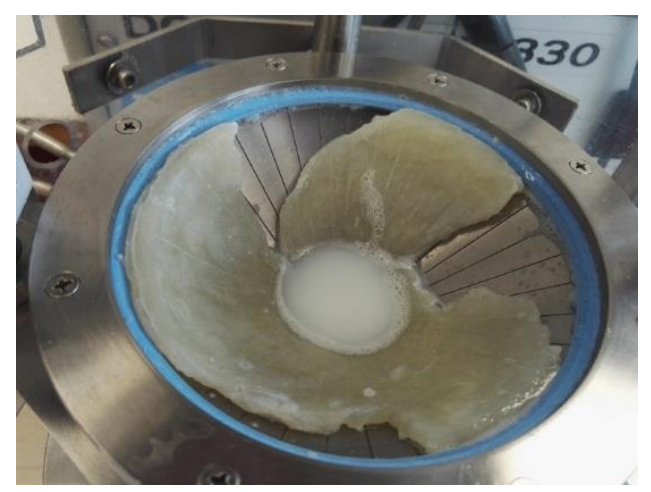

Fig. 4A -1 Sheared calcium caseinate dispersion at $50 \mathrm{rpm}$ for $5 \mathrm{~min}$. The dispersion was pre-heated at $130^{\circ} \mathrm{C}$ for $3 \mathrm{~min}$ and cooled down to room temperature before shearing. 


\section{Chapter 5 .}

\section{Maltodextrin promotes calcium caseinate fibre formation through air inclusion}

This chapter has been published as Wang, Z., Birgit, L. B., Boom, R., \& van der Goot, A. J. (2019). Maltodextrin promotes calcium caseinate fibre formation through air inclusion. Food Hydrocolloids, 95, 143-151. 


\begin{abstract}
Commercial calcium caseinate is available as spray-dried and roller-dried powder. Shearing a dense spray-dried calcium caseinate dispersion gives rise to a fibrous material, whereas shearing dense roller-dried calcium caseinate yields a layered material with only slight anisotropy in mechanical strength. The addition of a polysaccharide phase in a continuous protein phase may lead to formation of fibrous structures after shearing, which is hypothesized to be a result of the elongation and orientation of the dispersed polysaccharide domains. We report the effect of the addition of maltodextrin to rollerdried calcium caseinate on structure formation. The strength of the material increased with the addition of maltodextrin, which is partly caused by the withdrawal of water from the caseinate phase towards the maltodextrin phase, leading to a higher local caseinate concentration. The anisotropy of fracture stress and fracture strain were enhanced with up to $5 \mathrm{wt} \%$ maltodextrin. The effect of maltodextrin on the mechanical anisotropy and fibrous appearance could be ascribed to the greater air incorporation as a result of the presence of maltodextrin.
\end{abstract}




\subsection{Introduction}

The formation of a fibrous morphology in protein materials is of interest for the production of analogues for meat and mozzarella cheese. Extrusion is the technique most commonly used to achieve this (Arêas, 1992; Cheftel et al., 1992). A decade ago, a novel technique based on well-defined shear flow was introduced to create a fibrous structure from a dense calcium caseinate dispersion (Manski, et al., 2007a). Subjecting an aqueous calcium caseinate dispersion to pure shear flow can result in the formation of a highly fibrous matrix. Additional heterogeneities, such as air bubbles or fat droplets, can enhance the fibrousness and the mechanical anisotropy of the calcium caseinate material (Manski et al., 2008; Tian et al., 2018; Wang et al., 2019a). Scanning electron microscopy (SEM) imaging revealed that the resulting fibres showed anisotropy on a scale of 100-200 nm, both with and without air bubbles or fat droplets, when prepared from spray-dried calcium caseinate (Manski et al., 2007, 2008; Wang et al., 2019a). Nevertheless, the anisotropy and the fibrous appearance was more pronounced when the material contained air.

Recently, we found that calcium caseinate that was dried using a roller dryer did not form a fibrous matrix, which is probably related to the more severe thermal history compared with spray-dried calcium caseinate. Therefore, we investigated whether we can induce fibrousness in this material using a different mechanism.

Earlier studies based on both extrusion and well-defined shear deformation concluded that phase separation induced by combining thermodynamically incompatible biopolymers, such as proteins and polysaccharides, can result in anisotropic properties (Cheftel et al., 1992; Dekkers et al., 2018; Dekkers et al., 2016; Grabowska et al., 2016; Oberg et al., 2015; Tolstoguzov, 2006; Yuryev et al., 1991). Based on those studies, we investigated the shear-induced structuring of a calcium caseinate system prepared from roller-dried calcium caseinate and maltodextrin. In the case of sodium caseinate, the addition of maltodextrin was shown to yield a phase-separated system (Manoj et al., 1996). It was also reported that the addition of maltodextrin promoted fibrousness in 
extruded soy protein isolate (Yuryev et al., 1991). In addition, maltodextrin has been used in cheese analogues to improve texture (Jack, 2016) and was applied as fat replacer to produce mozzarella cheese from skimmed milk (Bhaskaracharya and Shah, 2001). In this study, the sheared materials were prepared in a shear cell device using dispersions consisting of $30 \mathrm{wt} \%$ calcium caseinate and varying concentrations of maltodextrin. These structures were investigated with confocal scanning laser microscopy (CSLM) and SEM. The entrapment of air in the material was studied with X-ray tomography (XRT). The tensile properties of the material were determined with a texture analyser. Furthermore, small deformation tests provided insights in the linear viscoelastic behaviour of the caseinate-maltodextrin dispersions.

\subsection{Materials and methods}

\subsubsection{Materials}

Roller-dried calcium caseinate powder was kindly provided by DMV International (Veghel, the Netherlands). The calcium caseinate powder (wet-base) contained $91.7 \mathrm{wt} \%$ protein and $1.2 \mathrm{wt} \%$ calcium according to the manufacturer's specifications. The dry matter content of calcium caseinate powder was $93.3 \mathrm{wt} \%$. Maltodextrin (Sigma-Aldrich, USA) with dextrose equivalent 16.5-19.5 was used. The dry matter content of maltodextrin was $96.6 \mathrm{wt} \%$. All the other chemicals used for microscopy preparation were purchased from Sigma Aldrich.

\subsubsection{Sample preparation in the shear cell device}

A caseinate-maltodextrin dispersion was prepared by manually mixing $30 \mathrm{~g}$ calcium caseinate powder with $70 \mathrm{~g}$ maltodextrin solution, with a spatula for $1 \mathrm{~min}$. First, maltodextrin solutions (70 g) were prepared by dissolving different amounts of maltodextrin $(1.5,3.5,5,7.5$ and $10 \mathrm{~g})$ in demineralized water. Two reference dispersions contained 30 and $35 \mathrm{wt} \%$ calcium caseinate powder without any maltodextrin.

Each dispersion was transferred to a pre-heated $\left(50^{\circ} \mathrm{C}\right)$ shear cell device with gap angle $2.5^{\circ}$ (Wageningen University, the Netherlands). This shear device was also used in 
previous studies (Wang et al., 2019a) and described in more details in an earlier publication (van der Zalm et al., 2012). The device consists of a rotating cone and a stationary cone that were both thermostatted with a heating and a cooling water bath. The shear treatment was carried out with a rotating speed of $150 \mathrm{rpm}$ for $5 \mathrm{~min}$. After stopping the shearing, the materials were cooled with a cooling water bath at $4^{\circ} \mathrm{C}$ before the cell was opened. Tensile tests were performed on materials within $1 \mathrm{~h}$ after discharge from the device. Other samples were stored at $-20^{\circ} \mathrm{C}$ until further analysis. Samples near to the rim ( $\sim 30-65 \mathrm{~mm}$ to the centre) were selected for tensile tests and further analysis.

\subsubsection{Tensile strength analysis}

A texture analyser (Instron Testing System, table model 5564) was used with a load cell of 100 N. A dog-bone-shaped mould was used to cut samples for tensile tests (Fig. 5-1). The samples were $15.2 \mathrm{~mm}$ long and $3.18 \mathrm{~mm}$ wide; the thickness varied between 4 and $6 \mathrm{~mm}$. The samples were taken parallel or perpendicular to the shear flow (based on the shear-vorticity plane).

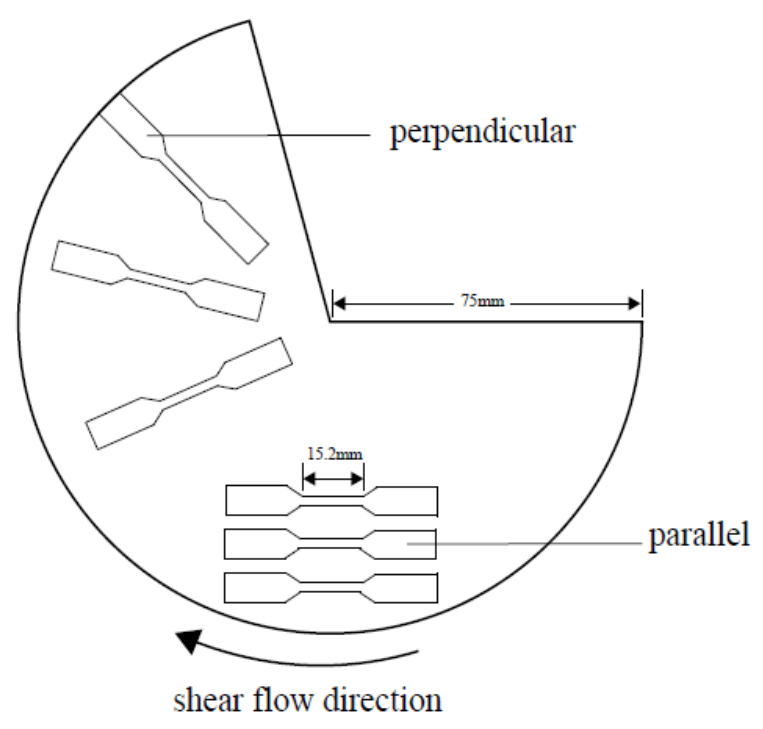

Fig 5-1. Schematic drawing of the sample which was taken from the device.

The tensile tests were conducted at a constant deformation speed of $3 \mathrm{~mm} \cdot \mathrm{s}^{-1}$. Grips with abrasive paper were used to prevent slipping in the grips during testing. At least three specimens per direction were measured. The results obtained from these tensile tests 
were depicted as force-displacement curves. The true stress $(\sigma, \mathrm{kPa})$ and Hencky strain $(\varepsilon,-)$ were calculated. The point at which a dramatic decrease in stress in the stressstrain curve was observed, was taken as the point of fracture, at which we measured the fracture stress $(\sigma, \mathrm{kPa})$ and fracture strain $(\varepsilon,-)$. Young's Modulus $(E, \mathrm{kPa})$ was calculated by applying a linear fit $(E=\sigma / \varepsilon)$ using the initial displacement, which was taken as the interval between 0 and $4.5 \mathrm{~mm}$ displacement. The apparent strain hardening coefficient $n$ was calculated by applying a power law fit $\left(\sigma=k \cdot \varepsilon^{n}\right)$ in the strain interval between $30 \%$ and $100 \%$ of fracture strain $\varepsilon$. When $n$ exceeds 1 , the sample exhibits strain hardening. $k$ is the strength coefficient, a mechanical property that indicates the stiffness of the material. The ratio of the averaged values measured in the parallel and perpendicular directions to the shear flow was used as an indication of the mechanical anisotropy of the samples.

\subsubsection{X-ray tomography}

The void fraction and geometry of air bubbles in the sheared materials were studied with X-ray tomography. Samples were scanned with a GE Phoenix v|tome|x m tomographer (General Electric, Wunstorf, Germany) set at $80 \mathrm{kV} / 90 \mu \mathrm{A}$. The system contains two X-ray sources; the $240 \mathrm{kV}$ micro-focus tube with a tungsten target was used. The images were recorded by a GE DXR detector array with $2024 \times 2024$ pixels (pixel size, $200 \mu \mathrm{m}$ ). The detector and object were located $815 \mathrm{~mm}$ and $28.55 \mathrm{~mm}$ from the X-ray source, respectively, resulting in a spatial resolution of $7.00 \mu \mathrm{m}$. A full scan consists of 750 projections over $360^{\circ}$. The first image was skipped; images 2-4 were averaged into one projection. GE reconstruction software (Wunstorf, Germany) was used to calculate the 3D structure via back projection. The 3D images were analysed using Avizo imaging software version 9.3.0. Three samples of each material were measured. The degree of deformation (Def) was defined as:

$$
D e f=(a-b) /(a+b)
$$

where $a$ is the length of a deformed air bubble parallel to the shear flow, and $b$ is the average width of the air bubble perpendicular to the shear flow (based on velocity 74 
gradient-shear flow plane and vorticity-shear flow plane). Def $=0$ represents a spherical air bubble; Def $=1$ represents an infinitely deformed air bubble.

\subsubsection{Confocal scanning laser microscopy}

Confocal scanning laser microscopy was used to observe the calcium caseinate and maltodextrin phase on a mesoscale. To locate the maltodextrin phase in caseinatemaltodextrin materials, maltodextrin was labelled with Rhodamine B isothiocyanate (RITC). The maltodextrin was covalently labelled using the method of De Belder and Granath (1973). Maltodextrin (10 g) was dispersed in dimethyl sulphoxide DMSO (100 $\mathrm{ml}$ ) for $15 \mathrm{~min}$ at room temperature. Pyridine (a few drops), RITC (0.1 g) and dibutyltin dilaurate $(200 \mathrm{mg})$ were added and the solution was stirred at $65^{\circ} \mathrm{C}$ for $2 \mathrm{~h}$. The maltodextrin was subsequently precipitated with ethanol $(95 \% \mathrm{v} / \mathrm{v})$. After filtration, the maltodextrin was washed several times with ethanol to remove the free dye, then once with absolute ethanol, followed with acetone and diethyl ether. The labelled maltodextrin was then dried overnight in an oven at $45^{\circ} \mathrm{C}$.

Rhodamine 110 was used to stain the calcium caseinate before sample preparation and shearing. Labelled and unlabelled maltodextrin powders were mixed well in the ratio of 1:4 based on weight before sample preparation in the shear cell device. Visually, maltodextrin labelled with RITC displayed a pink colour; calcium caseinate stained with Rhodamine 110 had a yellow colour. We checked that partly labelling maltodextrin had no discernible difference on shear-induced structure formation.

After shearing and cooling, the materials were cut into thin slices with a razor blade parallel to the shear flow, resulting in the velocity gradient-shear flow plane. The samples were transferred to 8-well chambered cover glasses (Ibidi, Germany) and visualized with LSM 510 (Zeiss, Oberkochen, Germany) using an Ar-ion laser (480 nm) and a HeNe laser $(543 \mathrm{~nm})$. For the protein phase, an emission filter of 505-530 $\mathrm{nm}$ was applied, whereas a 560-615 nm filter was used for the maltodextrin phase. Images were taken with a $20 \times$ dry objective (N.A. 0.3, zoom). Image analysis with Matlab was also performed to quantify the surface area fraction of the maltodextrin domains in the CSLM images. The surface 
area of air bubbles was excluded in the image analysis. Three images in each part were analysed.

\subsubsection{Compositional analyses}

Based on visual observation of the material with $10 \mathrm{wt} \%$ maltodextrin, its calcium caseinate and maltodextrin content could vary from the rim to the centre of the device. To confirm this, the moisture and protein content were measured. Samples were taken from different locations in the material. The moisture content was determined by drying the sample in an oven at $105^{\circ} \mathrm{C}$ overnight. The protein content of the pulverised dry samples was measured using Dumas analysis (Nitrogen analyser, FlashEA1112 series, Thermo Scientific, Interscience). A conversion factor of $\mathrm{N} \times 6.25$ was used.

\subsubsection{Scanning electron microscopy}

The sheared materials were torn apart manually along the shear flow direction $(\sim 2 \mathrm{~mm}$, based on the shear flow-vorticity plane), and rinsed with demineralized water. Subsequently, the samples were fixed in a glutaraldehyde solution $(2.5 \% \mathrm{v} / \mathrm{v})$ for $1 \mathrm{~h}$ and dehydrated in a graded series of ethanol (10\%, 30\%, 50\%, 70\%, 90\% and 100\% v/v, 20 min each). Critical point drying with carbon dioxide (CPD 020, Balzers, Liechtenstein) was performed. The dried samples were then glued to sample holders using conductive carbon cement (Leit-C, Neubauer Chemicalien, Germany), and sputter coated with $15 \mathrm{~nm}$ platinum (JFC 1200, JEOL, Japan). The surface was examined with a field emission scanning electron microscope (Magellan 400, FEI, Eindhoven, the Netherlands) at ambient temperature at a working distance of 4-8 $\mathrm{mm}$, with secondary electron detection at $2 \mathrm{kV}$ and $6.3 \mathrm{pA}$.

\subsubsection{Rheology}

Mixtures of $30 \mathrm{wt} \%$ calcium caseinate and maltodextrin (0-10 wt\%) were prepared at $70^{\circ} \mathrm{C}$ for $15 \mathrm{~min}$ to obtain homogeneous dispersions. Care was taken to remove any air. The mixtures were kept at $50^{\circ} \mathrm{C}$ before the rheological measurements. Strain sweep measurements within a strain range of $0.1-100 \%$ were performed to determine the linear 
viscoelastic region at $50^{\circ} \mathrm{C}$ using a Paar MCR 301 rheometer (Anton Paar, Graz, Austria) with serrated parallel plate geometry (diameter $25 \mathrm{~mm}$, gap $1 \mathrm{~mm}$ ). Frequency sweeps were performed at a constant strain of $1 \%$ (based on strain amplitude sweeps performed at $6.28 \mathrm{rad} / \mathrm{s}$ ) within an angular frequency $(\omega)$ range of $0.1-62.8 \mathrm{rad} / \mathrm{s}$. The samples were covered with silicone oil to reduce evaporation and rested for $1 \mathrm{~min}$ before testing. The measurement was also conducted with a $30 \mathrm{wt} \%$ maltodextrin solution.

\subsection{Results}

\subsubsection{Macrostructure}

Fig. 5-2 shows the materials obtained after shearing $30 \mathrm{wt} \%$ calcium caseinate with varying maltodextrin concentrations (0-10 wt\%). With only calcium caseinate (30 or 35 wt\%) or $1.5 \mathrm{wt} \%$ maltodextrin in $30 \mathrm{wt} \%$ calcium caseinate dispersion, a layered material was obtained after shearing (Fig. 5-52A). Those materials did not show any detached fibres on tearing. Calcium caseinate dispersions containing more maltodextrin (3.5 and 5 wt\%) yielded fibrous materials after shearing, demonstrated by isolated thin fibres after tearing (Fig. 5-2B,C). Addition of $7.5 \mathrm{wt} \%$ maltodextrin resulted in a fibrous material with some disrupted areas near the rim of the device (Fig. 5-2D). Addition of $10 \mathrm{wt} \%$ maltodextrin yielded damaged material near the rim and a fibrous material near the centre of the device (Fig. 5-2E). To allow a fair comparison of all materials, we took samples near the rim ( $\sim 30-65 \mathrm{~mm}$ from the centre) for further analysis. 

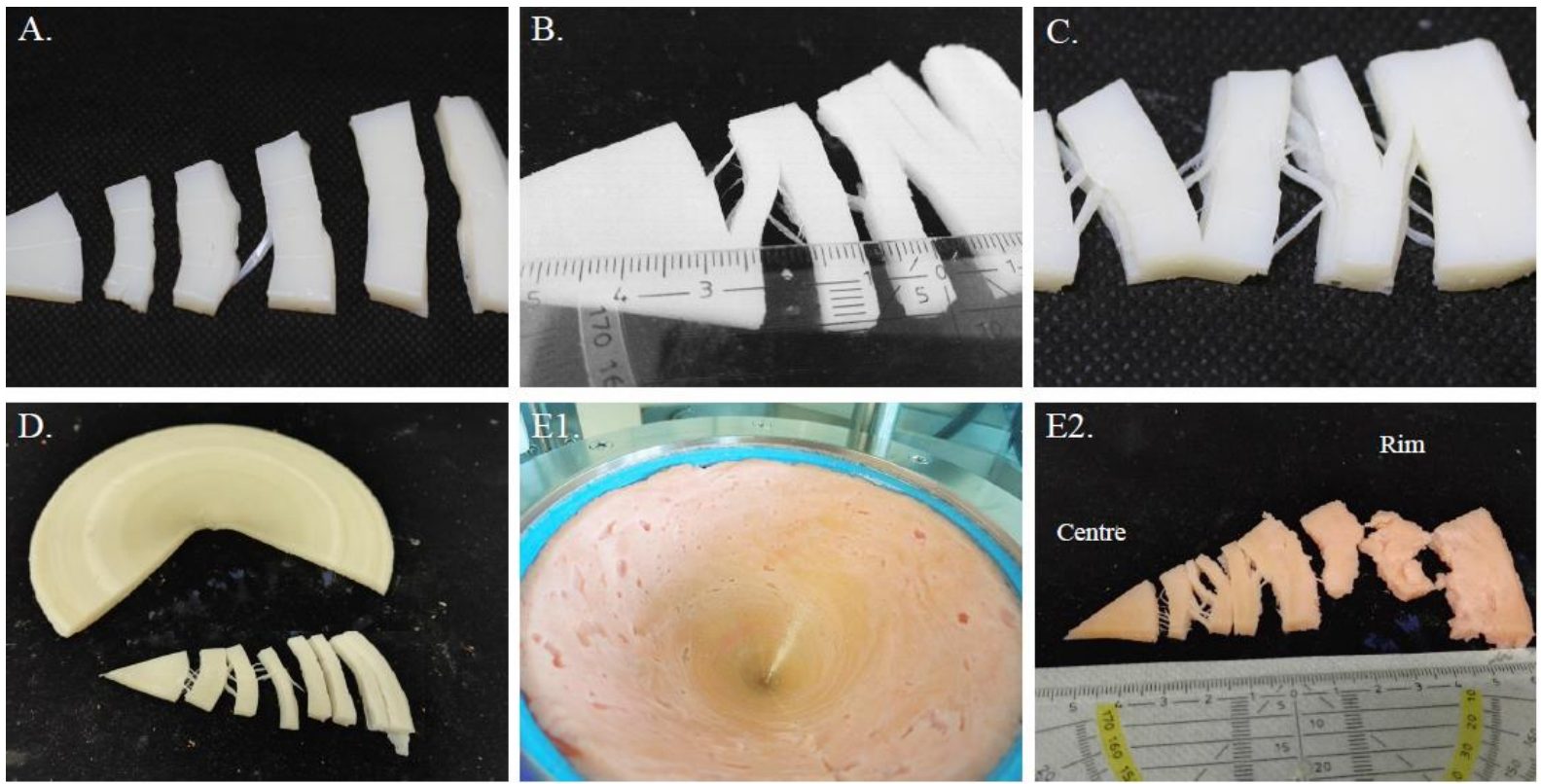

Fig. 5-2. Macrostructure of sheared $30 \mathrm{wt} \%$ calcium caseinate dispersions (A) without and with (B) $3.5 \mathrm{wt} \%$, (C) $5 \mathrm{wt} \%$, (D) $7.5 \mathrm{wt} \%$ and (E) $10 \mathrm{wt} \%$ maltodextrin. (D) The calcium caseinate (yellow) was stained with Rhodamine 110. (E) The maltodextrin (pink) and calcium caseinate (yellow) were stained with fluorescent dyes before shearing.

\subsubsection{Mesostructure}

CSLM images in Fig. 5-3 reveal the mesostructure of (un)sheared calcium caseinatemaltodextrin dispersions. The samples with 5 and $10 \mathrm{wt} \%$ maltodextrin were chosen because their macrostructures are clearly different compared with 30 wt\% calcium caseinate without maltodextrin (Fig. 5-2). In the absence of shearing (Fig. 5-3A), the sample with $5 \mathrm{wt} \%$ maltodextrin contained large maltodextrin domains (red), as well as air bubbles (black), dispersed in the calcium caseinate phase (yellow/green). After shearing (Fig. 5-3B), the maltodextrin domains appeared as smaller domains with irregular shape, and air bubbles were deformed in the shear flow direction. The surfaces of the air bubbles are surrounded by the maltodextrin phase, not the caseinate phase. No systematic alignment and orientation of the maltodextrin domains were observed in sheared materials. A high concentration of maltodextrin (10 wt\%) resulted in a partially damaged material (Fig. 5-2E). The CSLM image showed that calcium caseinate was the continuous phase in the fibrous sample near the centre of the device (Fig. 5-3C); the 
damaged sample near the rim had a bicontinuous structure (Fig. 5-3D). Image analysis showed that this disrupted structure contained more maltodextrin (area fraction of $52 \pm 3 \%$ ) than the fibrous structure near the centre (area fraction of $40 \pm 4 \%$ ). Thus, the calcium caseinate and maltodextrin content in this material is location dependent. This was further shown by determining the calcium caseinate and maltodextrin content at different locations (Fig. 5-A1). For the samples close to the centre (5-20 mm), the mass ratio between calcium caseinate and maltodextrin was 4.8 , whereas it was approximately 2.4 for the sample near the rim (55-75 mm). This macroscopic migration might be related to the phenomenon observed by Peighambardoust et al. (2008) in wheat dough under a curvilinear shear flow. A clear macro-separation was obtained between gluten and starch using the same shearing device as we used here (van der Zalm et al., 2012).

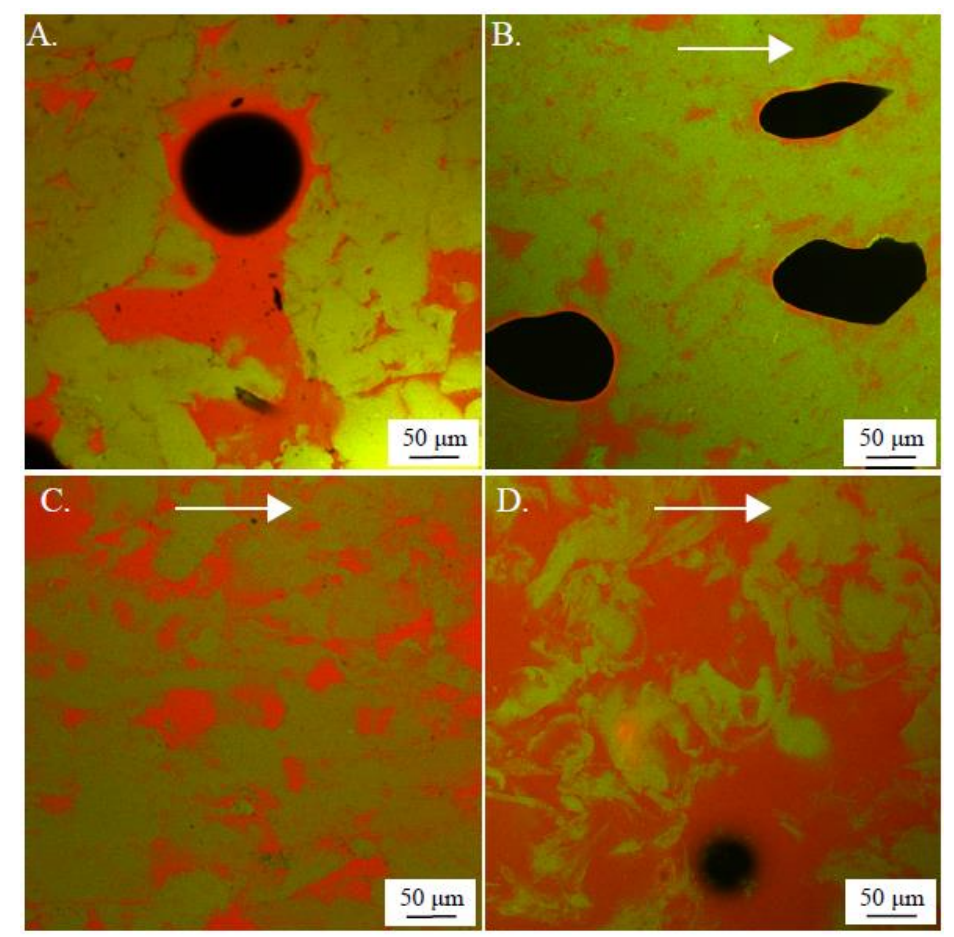

Fig. 5-3. CSLM images of materials containing $30 \mathrm{wt} \%$ calcium caseinate and $5 \mathrm{wt} \%$ maltodextrin: (A) before shearing ( $0 \mathrm{rpm}, 5 \mathrm{~min}$ ) and (B) after shearing at $150 \mathrm{rpm}$ for 5 min. CSLM images of the sheared material contained $30 \mathrm{wt} \%$ calcium caseinate and $10 \mathrm{wt} \%$ maltodextrin near the centre (C) and the rim (D) after shearing at $150 \mathrm{rpm}$ for $5 \mathrm{~min}$. Yellow/green indicates calcium caseinate; red indicates maltodextrin. The white arrow represents the shear flow direction. 
The volume fraction of air in (un)sheared materials could be measured with XRT (Fig. 54 and 5-5). The reference materials, consisting of 30 and $35 \mathrm{wt} \%$ calcium caseinate without maltodextrin, contained $2.3 \%$ and $1.7 \%(\mathrm{v} / \mathrm{v})$ air. The addition of $1.5 \mathrm{wt} \%$ maltodextrin had no significant effect on the void fraction $(2.7 \%, \mathrm{v} / \mathrm{v})$ compared with pure calcium caseinate material. In these samples with a low void fraction, air was mainly present as small air bubbles $\left(\leq 0.01 \mathrm{~mm}^{3}\right)$. The addition of $3.5 \mathrm{wt} \%$ maltodextrin increased the void fraction to $7.6 \%(\mathrm{v} / \mathrm{v})$ in the material. The material with $5 \mathrm{wt} \%$ maltodextrin had the highest void fraction $(11.0 \%, \mathrm{v} / \mathrm{v})$. These materials with higher void fraction mainly contained large air bubbles $\left(>0.01 \mathrm{~mm}^{3}\right)$. The addition of more maltodextrin (7.5 or $10 \mathrm{wt} \%$ ) resulted in a lower void fraction. These materials contained a lower fraction of large air bubbles $\left(>0.1 \mathrm{~mm}^{3}\right)$ than the materials with $5 \mathrm{wt} \%$ maltodextrin. Thus, the addition of maltodextrin increases the amount of air in sheared materials with an optimum at $5 \mathrm{wt} \%$. Image analyses based on XRT data showed that small air bubbles (Def $=0.0-0.1$ ) were not deformed, whereas large air bubbles were deformed (Def $=0.10-0.65$ ) and aligned in the shear flow direction (Fig. 5-4).
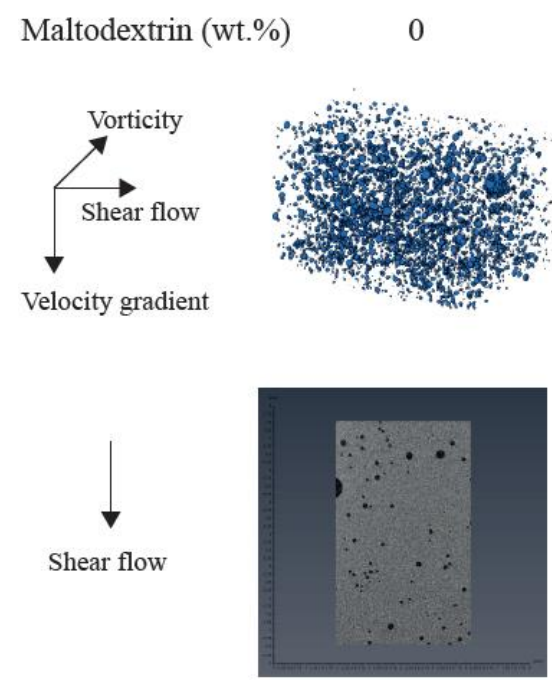

3.5
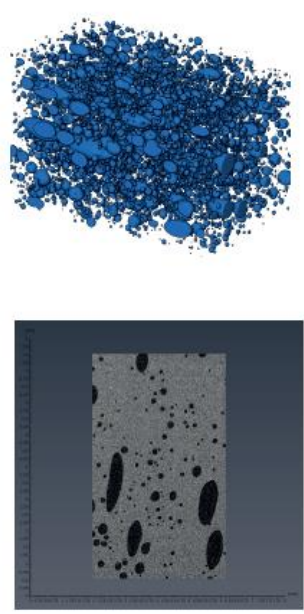
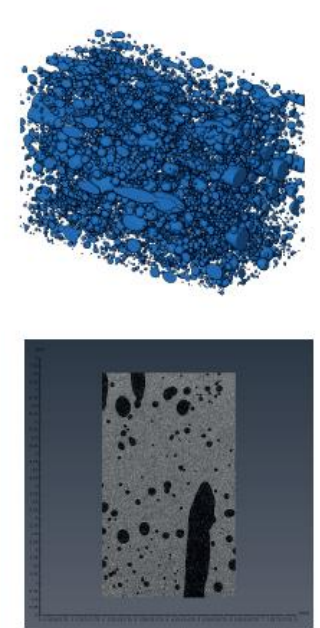

7.5
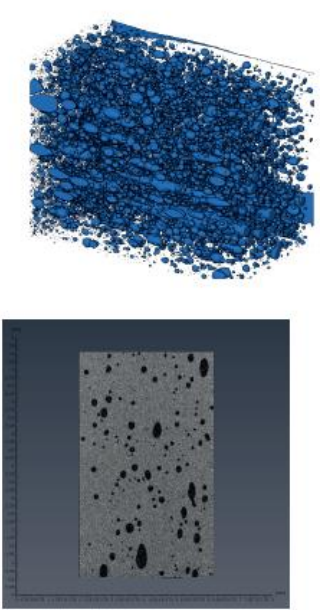

Fig. 5-4. XRT images of air bubble morphology in sheared materials containing $30 \mathrm{wt} \%$ calcium caseinate and various concentrations of maltodextrin. All the materials were obtained after shearing at $150 \mathrm{rpm}$ for $5 \mathrm{~min}$. Blue colour represents air bubbles in threedimensional images; black colour represents air bubbles in two-dimensional images. 


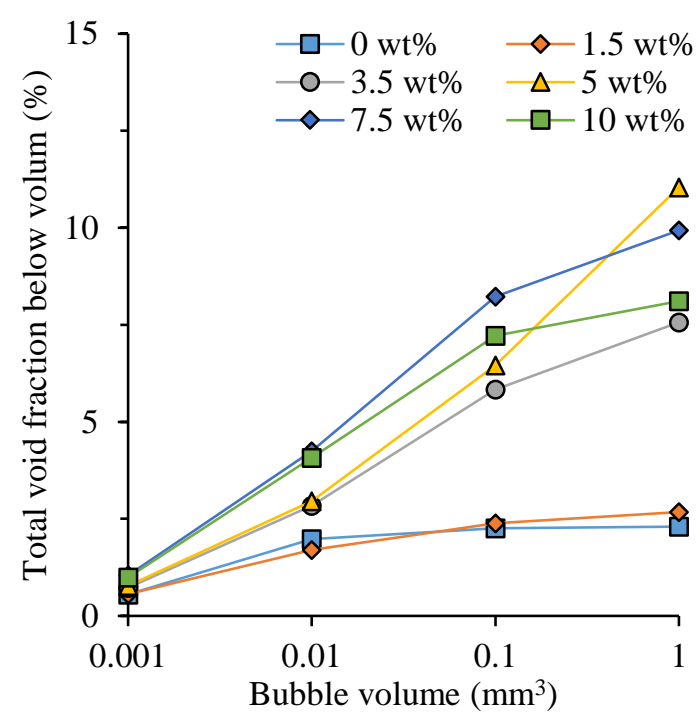

Fig. 5-5. Overall cumulative void fractions (as the void fraction below the bubble volume in $\mathrm{mm}^{3}$ ).

\subsubsection{Microstructure}

The SEM images in Fig. 5-6 show the microstructure of sheared material without maltodextrin and with $5 \mathrm{wt} \%$ maltodextrin. The latter sample has the most pronounced fibrous character (Fig. 5-2C), which has potential to show structural anisotropy at submicrometre length scale. In the absence of maltodextrin (Fig. 5-6A), non-oriented, spherical protein particles $(\sim 100-200 \mathrm{~nm})$ were observed. After the addition of maltodextrin (Fig. 5-6B), no protein alignment was obtained at this scale, which is accordance with the results reported by Yuryev et al., (1991) who showed that the fibrous structure of soy protein isolate-maltodextrin extrudates did not result from the orientation of macromolecular chains in the flow direction. However, protein alignment was shown in previous studies on shear-induced calcium caseinate fibre formation (Manski et al., 2008, 2007a; Wang et al., 2019a). Obviously, spray-dried calcium caseinate allows orientation at small scale, whereas the roller-dried one does not have this property. 


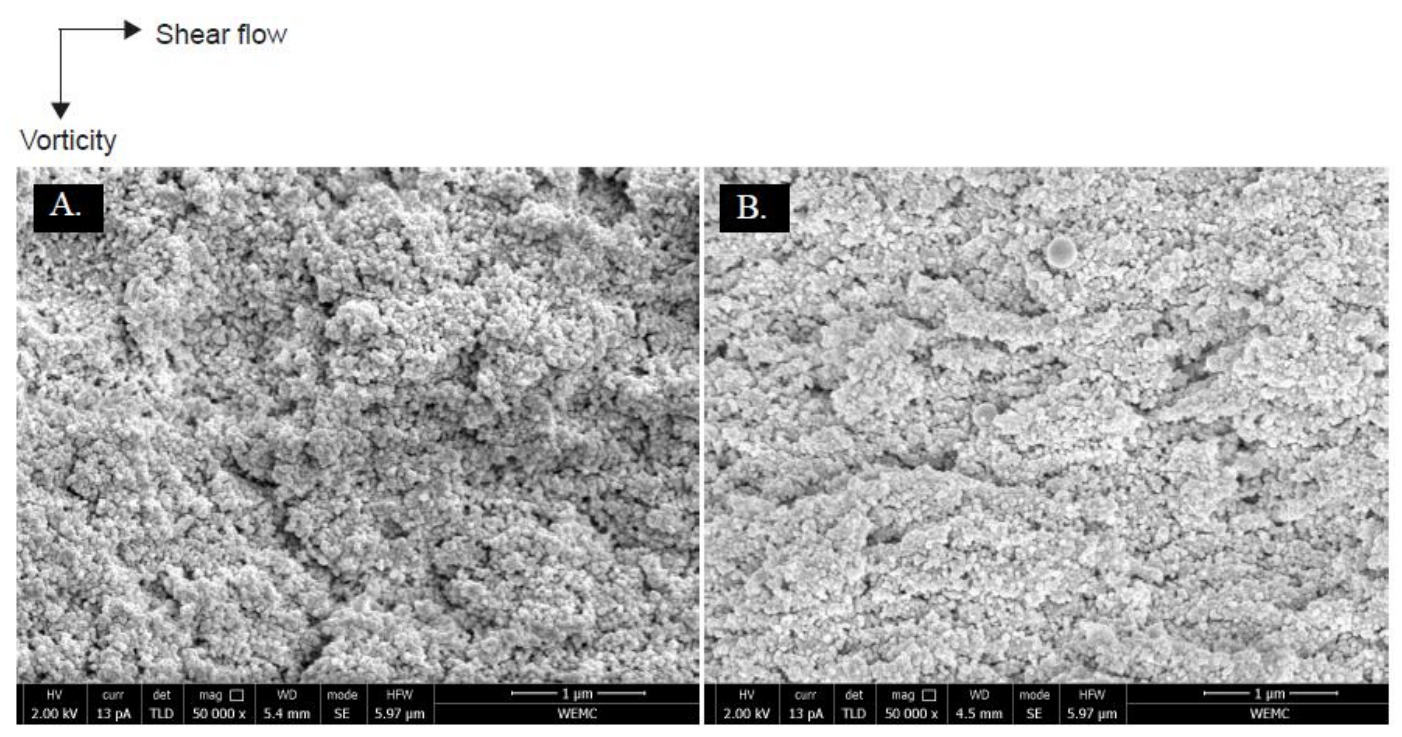

Fig. 5-6. SEM images of fractured surface parallel to the shear flow direction of the sheared material (A) without maltodextrin and (B) with $5 \mathrm{wt} \%$ maltodextrin. Scale bars indicate $1 \mu \mathrm{m}$.

\subsubsection{Tensile properties}

The addition of maltodextrin promoted fibre formation on macroscale (Fig. 5-2). We investigated the tensile properties to confirm the anisotropy. Tensile tests were performed parallel and perpendicular to the shear flow, and their ratio was used as an index for anisotropy. This anisotropy index of fracture stress $\sigma$ and strain $\varepsilon$ increased with the addition of maltodextrin up to $5 \mathrm{wt} \%$ and then decreased again for the material with $7.5 \mathrm{wt} \%$ maltodextrin (Fig. 5-7A, B). The material with $10 \%$ maltodextrin did not have sufficient coherence to be subjected to a tensile test. We compared the results with a sample that consisted of $35 \mathrm{wt} \%$ calcium caseinate, with a similar dry matter content as the material with $5 \mathrm{wt} \%$ maltodextrin. This sample showed anisotropy with an index of 1.5 for $\sigma$ and 1.3 for $\varepsilon$. Those values are similar to values for the $30 \mathrm{wt} \%$ caseinate material, but are much lower than the material with $5 \mathrm{wt} \%$ maltodextrin (3.3 for $\sigma$ and 2.3 for $\varepsilon$ ). This confirms that the addition of maltodextrin enhances the mechanical anisotropy. 

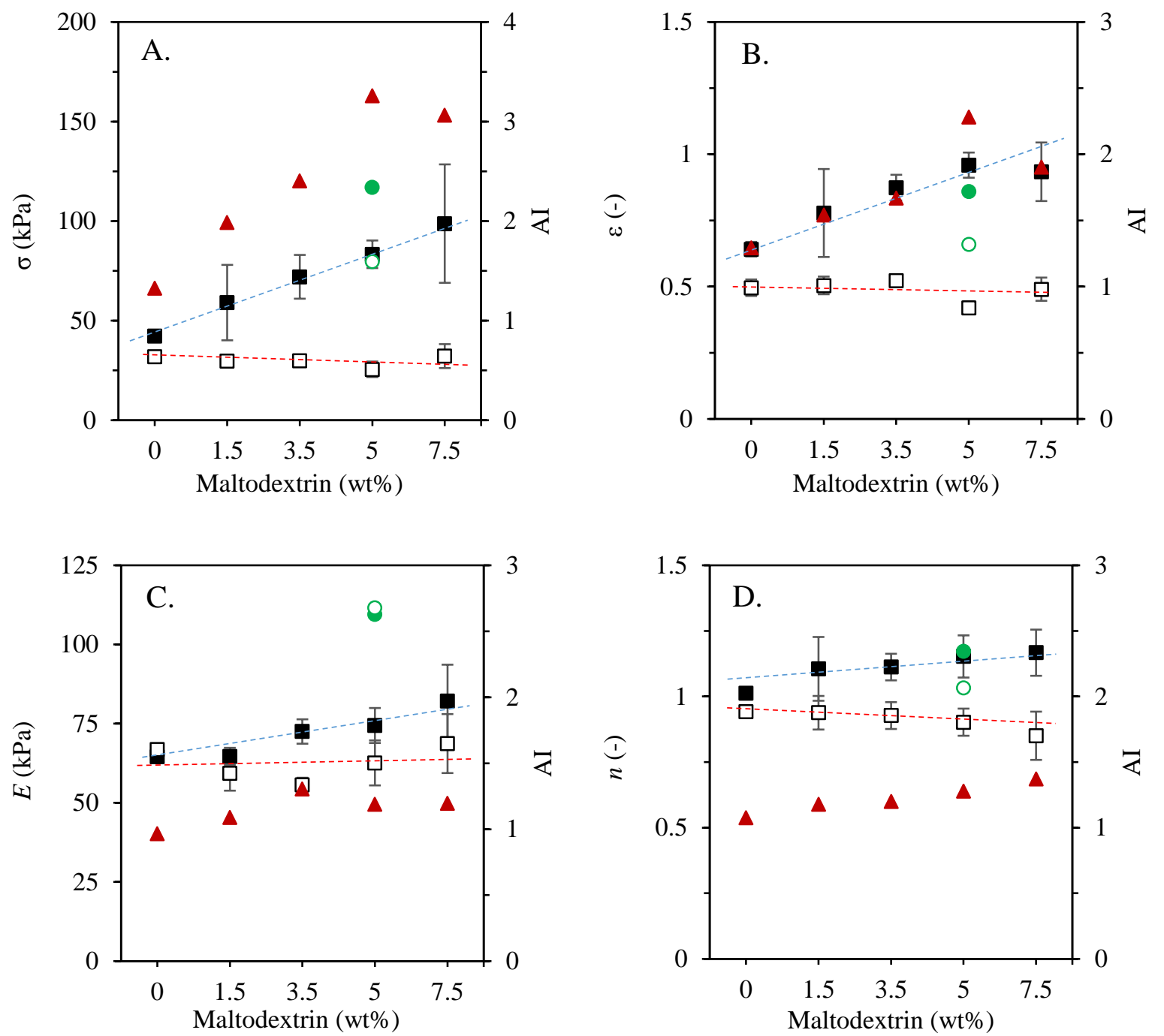

Fig. 5-7. Tensile properties: (A) fracture stress $\sigma$, (B) fracture strain $\varepsilon$, (C) Young's modulus $E$ and (D) apparent strain hardening coefficient $n$ as a function of maltodextrin concentration in the sheared calcium caseinate-maltodextrin materials, deformed parallel ( $\square$ ) and perpendicular $(\square)$ to the shear direction. The error bars for the mechanical properties are \pm standard deviation; where not visible, they were smaller than the marker used. The anisotropy index $\mathrm{AI}(\boldsymbol{\Delta})$ is the ratio between the average values measured in the parallel and perpendicular directions. The tensile properties of 35 wt\% calcium caseinate material were also measured in the parallel (O) and perpendicular direction $(O)$. The dashed lines were added to guide the eye. 
In the case of $30 \mathrm{wt} \%$ calcium caseinate without maltodextrin, the value for $\sigma$ and $\varepsilon$ in the parallel direction $(42 \mathrm{kPa}, 0.64)$ to the shear flow were only slightly higher than those in the perpendicular direction $(32 \mathrm{kPa}, 0.50)$. The strength in the parallel direction increased with the addition of maltodextrin. The material with $7.5 \mathrm{wt} \%$ maltodextrin showed large standard deviations in $\sigma$ and $\varepsilon$. We link these large variations to the observation that maltodextrin domains were unevenly distributed in the material; some of these domains were similar in size to the tensile bars used. In the perpendicular direction, no significant difference was found with the addition of maltodextrin. This is consistent with a layered or fibrous structure, resulting in continuity of the caseinate matrix in the parallel direction, whereas it is interrupted by maltodextrin in the perpendicular direction. The calcium caseinate-maltodextrin materials exhibited no significant anisotropy in Young's modulus and strain hardening (Fig. 5-7C, D), which could be due to the lack of structural anisotropy on a microscale (Fig. 5-6).

\subsubsection{Rheological properties}

Strain frequency sweeps were conducted with calcium caseinate-maltodextrin dispersions that were well mixed; care was taken to remove any air. Fig. 5-8A shows that the addition of maltodextrin to calcium caseinate resulted in increased value of the storage $\left(G^{\prime}\right)$ and loss moduli $\left(G^{\prime}\right)$. The addition of $5 \mathrm{wt} \%$ maltodextrin to a $30 \mathrm{wt} \%$ calcium caseinate dispersion resulted in higher values for both moduli compared with the $35 \mathrm{wt} \%$ calcium caseinate dispersion. All dispersions had a $G^{\prime}$ and $G^{\prime \prime}$ crossover, except for the dispersion with $10 \mathrm{wt} \%$ maltodextrin (data not shown). Here, the storage modules $G^{\prime}$ was already higher than the loss modules $G^{\prime \prime}$ at the lowest frequency measured which suggests crossover at a lower frequency than $0.1 \mathrm{rad} / \mathrm{s}$. In the case of a $30 \mathrm{wt} \%$ maltodextrin solution, both moduli were much lower than $30 \mathrm{wt} \%$ calcium caseinate dispersion (data not shown). Moreover, its $G^{\prime}$ values were much smaller than the $G^{\prime \prime}$ values throughout the frequency range measured, which indicates a fluid behaviour.

The inverse of the angular frequency $(\omega)$ at the crossover is defined as the relaxation time $(\tau)$. This relaxation time increases exponentially with the addition of maltodextrin (Fig. 
5-8B). The addition of $5 \mathrm{wt} \%$ maltodextrin to $30 \mathrm{wt} \%$ calcium caseinate dispersion resulted in a higher $\tau$ value compared with the $35 \mathrm{wt} \%$ calcium caseinate dispersion. Thus, the dispersion with maltodextrin behaved like a calcium caseinate dispersion with a higher concentration. Such a higher apparent concentration could be a result of water withdrawal from the caseinate, into the maltodextrin phase. The phase shift, The phase shift, expressed as $\tan \delta$ (equal to $G^{\prime \prime} / G^{\prime}$ ), decreased with the maltodextrin concentration throughout the frequency range measured, indicating that the dispersions are more solidlike with the addition of maltodextrin (Fig. 5-8C).
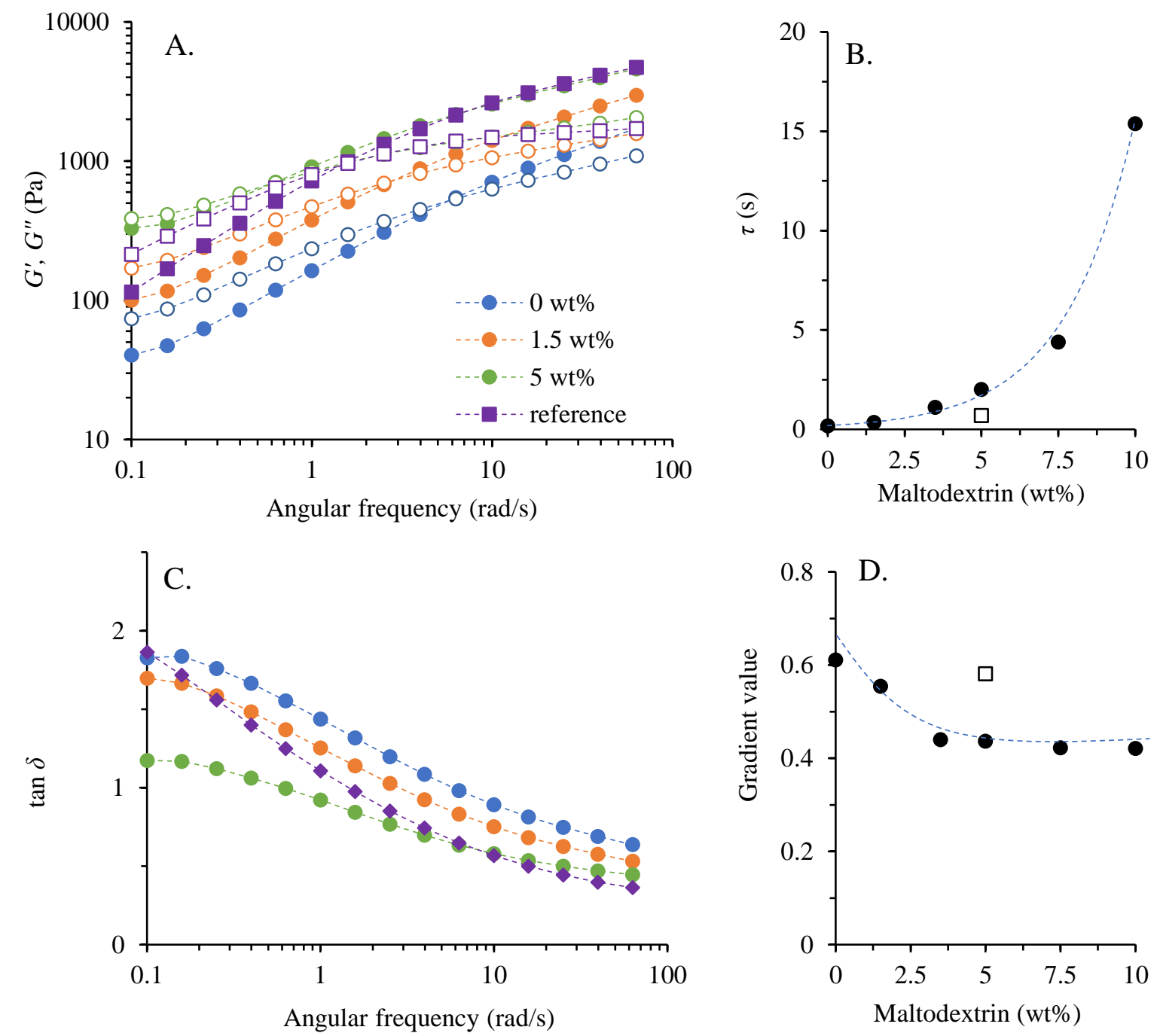

Fig. 5.8. (A) Storage modulus $G^{\prime}$ (closed circles) and loss modulus $G^{\prime \prime}$ (open circles) as a function of angular frequency of calcium caseinate-maltodextrin dispersions. (B) Relaxation time as a function of maltodextrin concentration. The line represents a fitted exponential. (C) Loss tangent ( $\tan \delta$ ) as a function of angular frequency. (D) The gradient 
values of $\mathrm{d}\left(\log G^{\Upsilon}\right) / \mathrm{d}(\log \omega)$ as a function of maltodextrin concentration. The open squares (B,C) represent the results for a $35 \mathrm{wt} \%$ calcium caseinate dispersion with no maltodextrin. The lines have been added to guide the eye.

The gradient value of $\mathrm{d}\left(\log G^{\prime}\right) / \mathrm{d}(\log \omega)$ provides useful information about the structure of the biopolymer (Ross-Murphy, 1995). When the gradient has a value approaching 0, the material behaves like a rubbery material, whereas a liquid flowing material has a gradient approaching 2 (Ferry, 1980). In the case of a three-dimensional network, the gradient value is expected to be near 0 , which is a characteristic of highly cross-linked material. Increasing the concentration of cross-linked material could cause the gradient value to decrease. Fig. 5-8D shows the effect of maltodextrin addition on the gradient value of $\mathrm{d}\left(\log G^{\prime}\right) / \mathrm{d}(\log \omega)$. This gradient value decreases with the addition of maltodextrin up to $3.5 \mathrm{wt} \%$, and then remains constant with higher concentrations. This indicates that the dispersions behave more as a solid after the addition of maltodextrin. The low gradient values of the dispersions with high maltodextrin concentrations (3.5$10 \mathrm{wt} \%$ ) coincide with high void fractions in the sheared materials (Fig. 5-5).

\subsection{Discussion}

The results show that the addition of maltodextrin promotes the mechanical anisotropy of calcium caseinate after well-defined deformation. The maltodextrin creates a separate phase next to the calcium caseinate phase. The resulting calcium caseinate-maltodextrin dispersion behaves more as a solid than the original caseinate matrix. The creation of this second phase (maltodextrin) also promotes withholding of air in the calcium caseinate matrix, which may play an important role in the anisotropy of the final materials, as shown previously (Tian et al., 2018; Wang et al., 2019b).

In extrusion, it is known that the addition of a separated polysaccharide phase leads to elongated, dispersed polysaccharide domains after processing, which then leads to a fibrous morphology of the final material and mechanical anisotropy on tearing (Cheftel et al., 1992; Tolstoguzov, 2006; Yuryev et al., 1991). A similar observation has been reported by for soy-gluten blends and soy-pectin blends in a device similar to that used in this 
study (Dekkers et al., 2018; Dekkers et al., 2016). One might expect therefore that the polysaccharide phase in the calcium caseinate-maltodextrin dispersion is also aligned. However, no evidence was found in the CSLM images that dispersed maltodextrin domains were aligned or oriented (Fig. 5-3).

There is a correlation between the void fraction and the fracture anisotropy of the materials (Fig. 5-9). Our earlier study on spray-dried calcium caseinate systems without maltodextrin indeed showed that air is important for mechanical anisotropy (Wang et al., 2019a, 2019b), probably through localized stress build-up. It was also found that deformation of air bubbles influences the mechanical anisotropy of processed materials, such as sheared soy-pectin materials (Dekkers et al., 2018) and whey-starch extrudates (Cho and Rizvi, 2009). In line with this, we also conclude that the anisotropy of calcium caseinate-maltodextrin materials originates from the presence of air bubbles. All air bubbles that were observed using CSLM, were embedded in maltodextrin, rather than in the caseinate phase (Fig. 5-3A, B). Therefore, we postulate that maltodextrin is responsible for the withholding of air in roller-dried calcium caseinate and thus promotes anisotropy through this mechanism. On shearing, the additional maltodextrin stabilizes the air bubbles by increasing the viscosity. However, the high viscosity of the continuous calcium caseinate phase induces the deformation and alignment of air bubbles (Fig. 5-4). This phase may be too viscous to allow air bubbles to migrate, which might explain why the air associates with the maltodextrin phase. In the case of higher addition of maltodextrin, it is likely that the volume fraction of the maltodextrin is higher, which could lead to enhanced coalescence and eventually phase inversion; which is consistent with the breakup of the matrix at high maltodextrin concentrations. The latter could be stimulated by the large differences in viscosity between the maltodextrin and calcium caseinate phase: the maltodextrin phase has a much lower viscosity than the calcium caseinate phase, and therefore has a tendency to invert to the continuous phase. Larger domains of maltodextrin further facilitate the escape of air from this phase, which explains the lower void fractions at higher maltodextrin concentrations. Shorter 
processing time could have prevented phase inversion, but then the anisotropic structure would not have been formed.

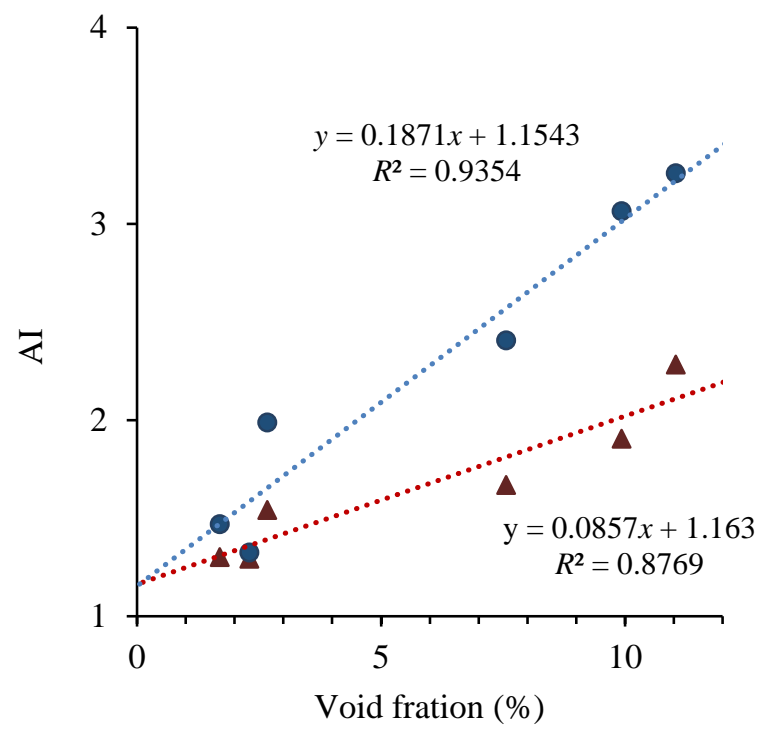

Fig. 5-9. Anisotropy in fracture stress (circles) and strain (triangles) (cf. Fig. 5-7A,B) as function of the overall void fraction (cf. Fig. 5-5).

\subsection{Conclusion}

A roller-dried calcium caseinate dispersion cannot be transformed into a fibrous material due to the heat treatment during powder production, but it gives a layered structure with only slight anisotropy after shearing. The addition of maltodextrin promotes the formation of fibre and enhances mechanical anisotropy. The effect of maltodextrin is twofold. (1) It resulted in a more solid-like dispersion due to a higher apparent protein concentration. Moreover, through the addition of maltodextrin, (2) air is held within in the dispersion, and we found a correlation between the fracture anisotropy and the void fraction. The bubbles were large and elongated, which is important for the fibrous appearance on a macroscale. Here, we found that the fibrous structure or mechanical anisotropy was a result of anisotropy on a mesoscale. In contrast to structured spraydried calcium caseinate, no protein alignment was observed at smaller length scale. At high maltodextrin concentrations, phase inversion occurs, leading to an inhomogeneous, disrupted material and less air incorporation. Clearly, there is an upper limit for the addition of maltodextrin. 


\section{Acknowledgements}

The authors would like to thank the China Scholarship Council (grant number 201406820015) for financial support, Friesland Campina for kindly supplying the protein ingredients, Anna Moller and Zulhaj Rizki for helping with the image analysis and Jarno Gieteling for technical support. 


\section{Appendix 5A}

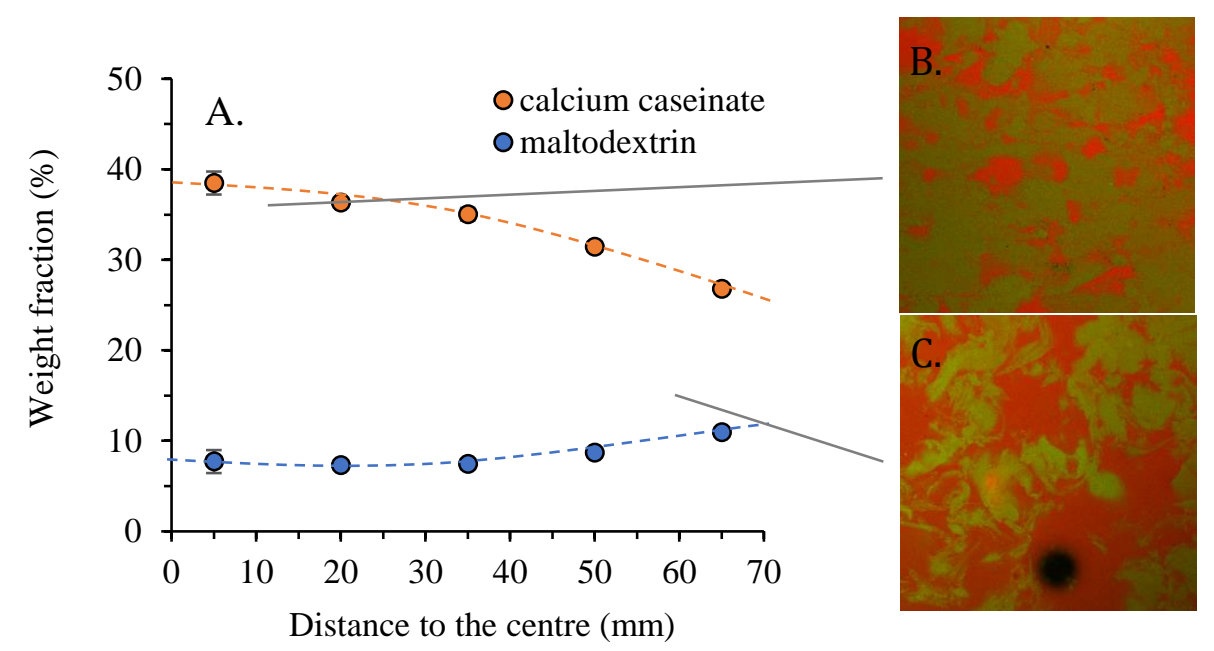

Fig. 5A -1. (A) Weight fraction of calcium caseinate and maltodextrin as a function of the sample location. The sheared sample contained $30 \mathrm{wt} \%$ calcium caseinate and $10 \mathrm{wt} \%$ maltodextrin. (B,C) CLSM images of the sample near the centre and the rim. 
Chapter 6

General discussion 


\subsection{Introduction}

The creation of fibrous structures within protein foods is of great relevance, but still not completely understood. Therefore, this thesis aimed to further understand the mechanisms of fibrous structure formation in dense calcium caseinate dispersions. This chapter summarises the main findings of this thesis, and discusses the intrinsic properties of calcium caseinate and the importance of air bubbles. The chapter is completed with a discussion on the potential of extrapolating the new insights for caseinate system towards plant-based protein materials.

\subsection{Main findings}

Concentrated calcium caseinate dispersions can be transformed into hierarchically structured, fibrous materials using well-defined shear deformation and concurrent enzymatic crosslinking by transglutaminase. This thesis identified that there is a parameter in this system that was hitherto not investigated, and which was found crucial: air holdup. Thus, we investigated the role of air bubbles on the microstructure and mechanical properties of the final material (Chapter 2). Although anisotropy is also obtained in the absence of entrapped air, the fibrous appearance and especially the mechanical anisotropy of the calcium caseinate materials was much more pronounced with dispersed air being present. Air was therefore considered as an additional phase causing the material to become tougher with strain hardening in the direction parallel to the fibres, and more susceptible to fracture upon stretching in the perpendicular direction. Chapter $\mathbf{3}$ further considers the relative importance of air bubbles and protein alignment on the mechanical anisotropy of calcium caseinate material. It is concluded that the deformation of air bubbles can only partly explain the mechanical anisotropy; the anisotropy of the protein phase is also important. It was further concluded that air bubbles have an influence on the protein phase behaviour during processing, thereby affecting the degree of anisotropy of the final material.

Calcium caseinate is commercial available in two types: roller-dried and spray-dried powder. Shearing spray-dried calcium caseinate dispersions resulted in fibrous materials, whereas shearing roller-dried calcium caseinate dispersions yielded layered materials with less mechanical anisotropy (Chapter 4). Those differences were attributed to the more intensive thermal treatment during roller drying. Heat-induced changes led to 
reduced susceptibility to enzymatic crosslinking through transglutaminase, as well as reduced interaction with $\mathrm{Ca}^{2+}$. The latter resulted in less solid-like behaviour of rollerdried calcium caseinate dispersions. Chapter 5 describes a method to make fibrous materials using roller-dried calcium caseinate with a different structuring mechanism. Fibrous materials were formed by adding maltodextrin to roller-dried calcium caseinate dispersions. The addition resulted in more solid-like behaviour and led to more air holdup in sheared materials. Here, we found that the fibrous appearance and mechanical anisotropy was a result of anisotropy on mesoscale. Moreover, no protein alignment was observed at a smaller length scale, which is in contrast when using spray-dried calcium caseinate.

Overall, the research established that fibrous structure formation under well-defined shear flow is enhanced by air bubbles. In case of spray-dried calcium caseinate, the high anisotropy is caused by a combination of the anisotropy in the protein phase and the additional effect of air. The effect of air is a result of air bubble alignment with air bubbles remaining mostly spherical. However, the presence of largely elongated air bubbles in an isotropic matrix could still induced fibrous appearance and mechanical anisotropy when using roller-dried calcium caseinate.

\subsection{Shear-induced fibre formation from spray-dried calcium} caseinate powder

Spray dried calcium caseinate powder was used to create hierarchically fibrous materials using well-defined shear deformation. The resulting material visually appeared highly fibrous when tearing along the direction of shear flow. A typical fibre diameter of $\sim 100-$ $200 \mathrm{~nm}$ was observed with scanning electron microscopy (SEM). Here, we summarise the main criteria for spray dried calcium caseinate powder as a suitable ingredient for fibrous structure formation.

\subsubsection{Protein: structural elements at small length scales}

The structuring of calcium caseinate is the result of a mechanism that can be described as directed self-assembly (Boom, 2011). Self-assembly is the spontaneous assembly of molecules into structural elements such as aggregates, fibrils or micelles through molecular association. Caseins in calcium caseinate tend to self-assemble into caseinate 
micelles (100-300 nm) with attractive interactions between micelles, due to the presence of $\mathrm{Ca}^{2+}$. The size of the micelles and the interactions between them are important factors influencing shear-induced structuring (Manski et al., 2007a). In case of calcium caseinate, these micelles are large enough to be aligned by shear force, while their interaction are strong enough to have them associated into strings, but sufficiently weak to avoid the formation of larger and inflexible aggregates. By concurrent enzymatic crosslinking with transglutaminase, the aligned aggregates can be fixed, effectively creating a fibrous structure (Fig. 6-1A).

Directed self-assembly as a mechanism for fibrous structure formation was also suggested in an earlier work, in which calcium caseinate and sodium caseinate were compared under shear deformation (Manski et al., 2007c). Shearing a $30 \mathrm{wt} \%$ sodium caseinate dispersion at similar conditions as calcium caseinate resulted in an isotropic material without any orientation at macroscopic scale. Sodium caseinate micelles are smaller (20-50 nm) and did not show a tendency to cluster, making sodium caseinate micelles unsusceptible to shear alignment. Moreover, the formation of fibrous structure in calcium caseinate was blocked by the addition of sodium triphosphate, which exchanges the $\mathrm{Ca}^{2+}$ bound to the caseins with $\mathrm{Na}^{+}$(Grabowska et al., 2012).

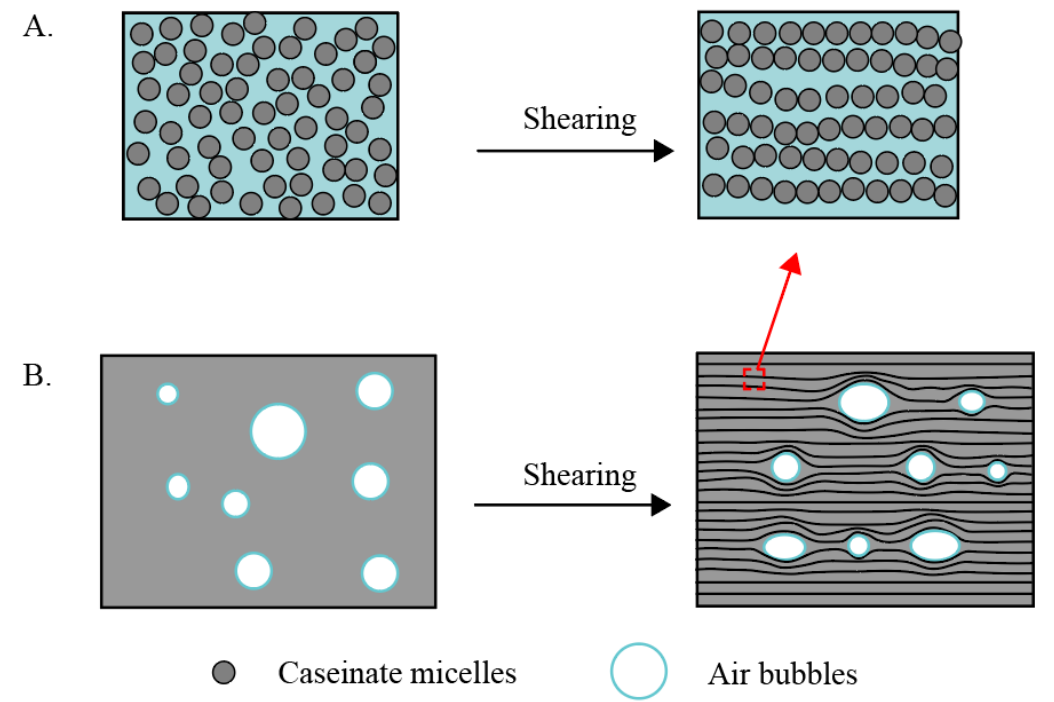

Fig. 6-1. Schematic picture on shear-induced fibrous structure from spray dried powder. The diameter of caseinate micelles is about $1 \mu \mathrm{m}(\mathrm{A})$; the diameter of air bubbles is about 100-1000 $\mu \mathrm{m}$ (B). 


\subsubsection{Air bubbles: structural elements at a microscale}

Air bubbles incorporated in calcium caseinate matrices greatly enhanced the fibrous nature and the mechanical anisotropy of the material (Fig. 6-1B). Air bubbles act as a weak phase in the perpendicular direction to the shear flow. As a result, the stress on their surface and especially near their locations with small curvature is concentrated, which makes the material more susceptible to fracture. In parallel direction to the shear flow, the incorporated air causes the material to become tougher (Chapter 2). In Chapter 3, the relative importance of air bubbles and caseinate (micelles) alignment on the mechanical anisotropy of calcium caseinate material was studied. The effect of air on the mechanical anisotropy of these fibrous materials was described with a load-bearing model. The anisotropy of the protein phase in the aerated materials was estimated and compared with the materials obtained from deaerated caseinate dispersions. It was concluded that the deformation of air bubbles as such can only partly explain the mechanical anisotropy; the anisotropy of the protein phase is still important. However, this matrix anisotropy is enhanced by the presence of air bubbles during shearing. The hypothesis is that the local differences in loss modulus, due to the combination of the air bubbles and the (local) strain hardening of the dispersion, lead to local differences in shear rate, thereby leading to the formation of bands or strings that ultimately form a fibrous material.

This effect of air bubbles on the matrix morphology is also significant at lower concentrations. $25 \mathrm{wt} \%$ calcium caseinate dispersions were prepared with and without air bubbles, and then sheared at $50{ }^{\circ} \mathrm{C}$ and $50 \mathrm{rpm}$ for $5 \mathrm{~min}$. The resulting material with air bubbles was visually anisotropic upon tearing along the shear flow direction (Fig. 6$2 \mathrm{~A})$. In the absence of air, the dispersion formed a sticky isotropic material, which could not be removed from the shear cell without breaking the material (Fig. 6-2B).

A.

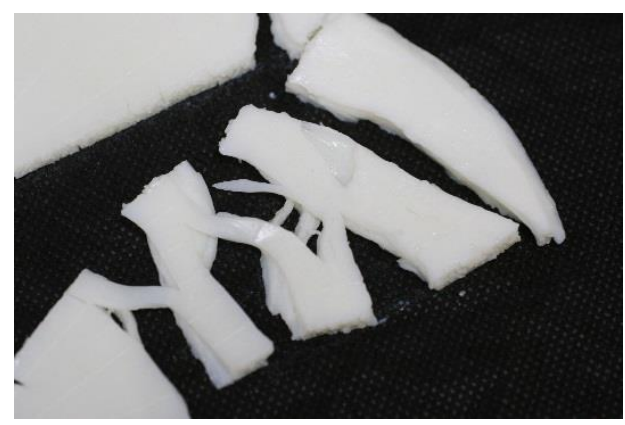

B.

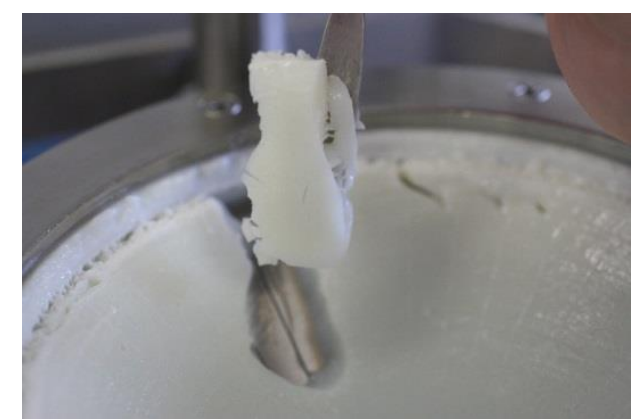

Fig. 6-2. Sheared 25 wt\% calcium caseinate dispersion (A) with and (B) without air bubbles at $50{ }^{\circ} \mathrm{C}$ and $50 \mathrm{rpm}$ for $5 \mathrm{~min}$. 


\subsubsection{Caseinate powder properties}

The processing history of calcium caseinate powder is of importance for its structuring potential (Chapter 4). Severe heat treatment induces aggregation of the caseins, resulting in larger aggregates when hydrated. A calcium caseinate dispersion with such large particles forms a layered material with slight mechanical anisotropy after shearing, which corresponds to the absence of protein alignment at submicron length scale. A second effect is the prevalence of $\mathrm{Ca}^{2+}$ in the caseinate: its associative effect is important for the formation of protein alignment at microscale. Hence, variations in the $\mathrm{Ca}^{2+}$ content will have an effect on the degree of anisotropy after shearing. Both effects could be important in explaining the variations on the tendency to form a fibrous structure, between powders with different processing history and between different batches with similar process history.

Spray dried calcium caseinate powder is hydrated more slowly than roller dried powder. Calcium caseinate powder (10 wt\%) was mixed with water at $50{ }^{\circ} \mathrm{C}$ for $5 \mathrm{~min}$. For spraydried caseinate, subsequent centrifugation $(18,400 \mathrm{~g}, 5 \mathrm{~min}), 68 \mathrm{wt} \%$ of calcium caseinate ended up in the pellet (Fig. 6-3). The particle in the sediment has a similar morphology and larger size distribution when compared to dry powder (Appendix 6A). In case of roller-dried powder, $14 \mathrm{wt} \%$ of calcium caseinate was in the sediment.

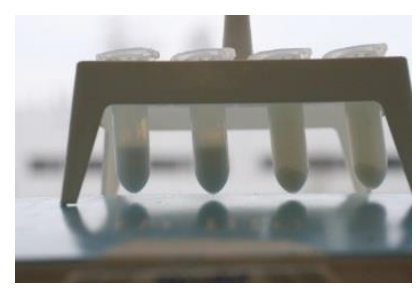

Fig 6-3. Spray-dried (left two) and roller-dried (right two) calcium caseinate dispersions (after 5 min mixing) after centrifugation at 18,400 $g$ for 5 min.

We noticed that slower hydration is positively correlated with the tendency of a dispersion to form fibre upon shearing. A $30 \mathrm{wt} \%$ dispersion after resting for some time resulted in a lower air holdup and less pronounced fibrousness after shearing (Chapter 2).

Subjecting a freshly prepared $20 \mathrm{wt} \%$ calcium caseinate dispersion to constant shear led to high initial shear stress, which then gradually decreased in approximately $2 \mathrm{~min}$, and finally remained constant (Fig. 6-4) The same dispersion that was allowed to hydrate 
overnight, showed constant shear stresses as a function of shear time, at a somewhat lower level than the final value with the fresh dispersion. This $20 \mathrm{wt} \%$ dispersion that was allowed to hydrate overnight, did not give any fibrousness after shearing; the freshly prepared dispersion did give some weak fibres. We therefore hypothesize that incomplete hydration results in an inhomogeneous material. This is supported by the larger shear stress obtained with the freshly prepared dispersion, which still contains a significant volume of particulate matter. As discussed before, some inhomogeneity may lead to the formation of shear bands, which will help the formation of a fibrous material. Of course there is balance here; if the powder cannot be sufficiently dissolved, then the inhomogeneity will be too large, and any incipient structure will be destroyed by the motion (and rotation) of the inhomogeneity. We confirmed this hypothesis by spray drying a batch of calcium caseinate using a lab-scale spray dryer at high air temperatures. We observed that the calcium caseinate obtained is difficult to disperse in water, and cannot be used for fibre formation in a shear cell.

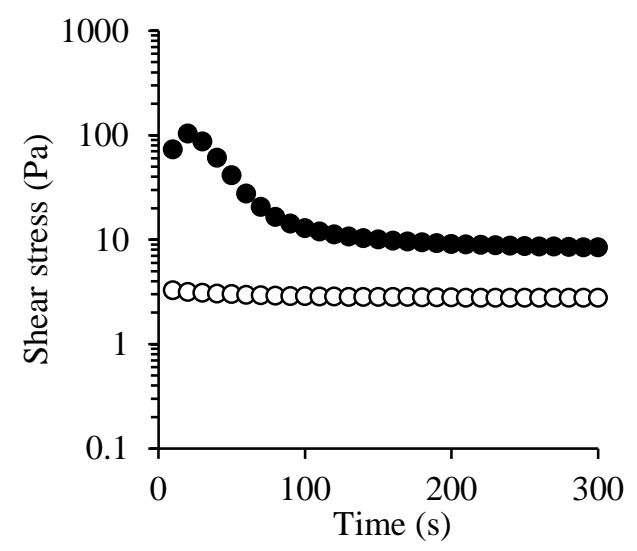

Fig 6.4. Shear stress as function of time for $20 \mathrm{wt} \%$ calcium caseinate dispersions at $50 \mathrm{~s}^{-}$ 1. Dispersions were prepared by roughly mixing calcium caseinate powder with water. Constant shear rate tests were performed on the dispersion immediately after sample preparation (O) and after storage at room temperature overnight (O).

\subsection{Mechanisms of shear-induced fibre formation}

This thesis describes two routes for structuring calcium caseinate. In case of spray-dried calcium caseinate, we made use of the intrinsic properties of the material. Shearing this dispersion leads to alignment at small length scale. Strain hardening behaviour prevents the loss of air that is introduced when mixing the porous spray dried particles with water. Roller-dried calcium caseinate does not possess these properties. The nature of the roller 
drying process implies that hardly any air is retained in the powder, leading to a very low air holdup in its aqueous dispersion. Shearing roller-dried calcium caseinate results in a layered material with only slight mechanical anisotropy (Chapter 4). The resulting material does not show protein alignments at small length scale, which we attribute to the large inhomogeneity caused by large particles $(2-300 \mu \mathrm{m})$. Those are thought to disrupt any structure created at smaller scale. A few large and deformed air bubbles still resulted in a layered appearance upon tearing the material.

The addition of maltodextrin induced fibre formation and more mechanical anisotropy (Chapter 5). The maltodextrin solution holds air, which is retained in the caseinatemaltodextrin dispersion. The interfaces between the two phases will stabilize the air bubbles. A second effect is that the water in the dispersion is unequally distributed over the maltodextrin phase and the caseinate phase. Preliminary experiments of water content in both phases revealed that maltodextrin absorbs more water, which makes the caseinate phase more concentrated. Indeed, the resulting calcium caseinate-maltodextrin dispersions behaved similarly to a caseinate dispersion with a higher concentration. This higher effective concentration also leads to a better stability and retention of the air bubbles. Upon shearing, stress is applied to both the calcium caseinate and the maltodextrin phase (Fig. 6-5). The dispersed air bubbles are deformed and aligned in the shear flow direction. When tearing the material, it breaks along the long side of the air bubbles, resulting in a fibrous appearance.

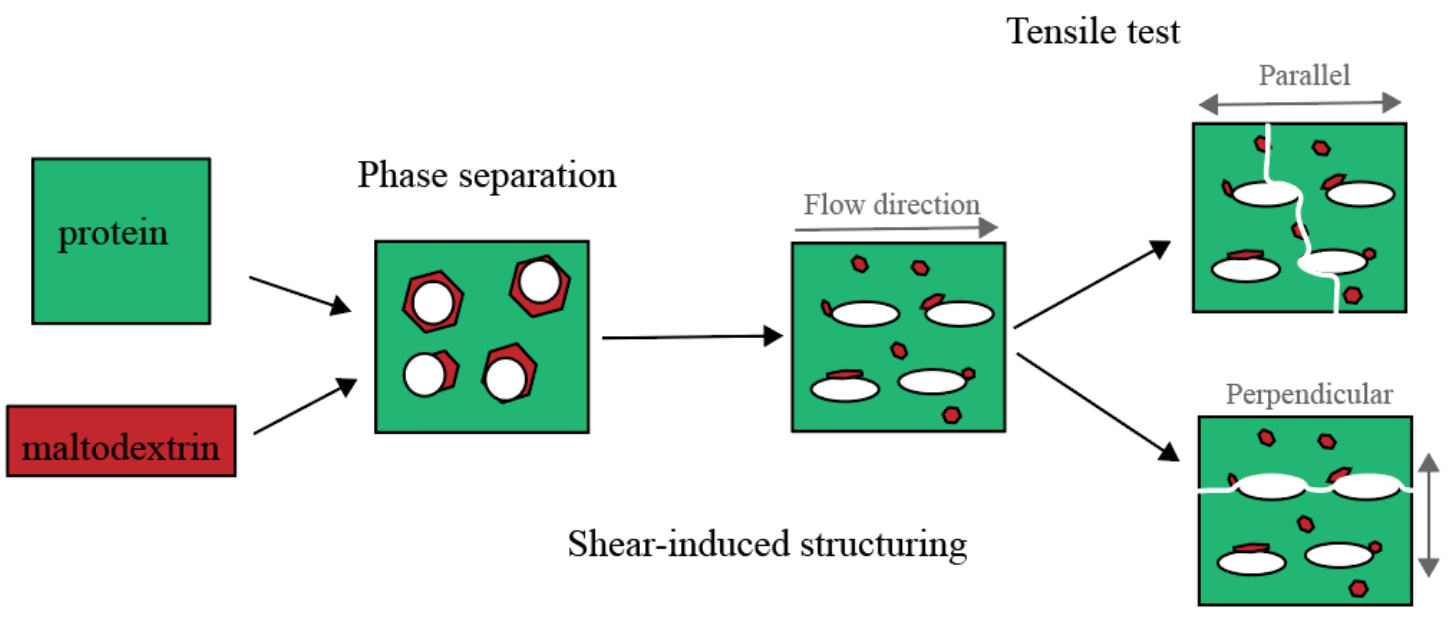

Fig. 6-5. Schematic picture of shear-induced structuring and fracture behaviour of the resulting material which was deformed in the parallel and perpendicular direction of shear flow. 
When the maltodextrin phase is not or is insufficiently added, the amount of air added is too limited, resulting in a layered, but not fibrous material. When adding too much maltodextrin, the volume fraction of maltodextrin phase is higher, leading to enhanced coalescence and eventually phase inversion. As a result, a broken material is obtained. Hence, the phase volume of maltodextrin is of importance for the final material.

\subsection{Structural analysis of shear-induced fibrous material}

Adequate structural analysis is important for quantification of the material properties. Different analytical techniques are necessary to determine the size and anisotropy on different length scales.

Till now, visual inspection of the material upon tearing has been used for a direct assessment of the macrostructure. However, this only gives a first and qualitative impression. The tensile test was used to quantify the mechanical anisotropy. Fibrous calcium caseinate materials have much higher fracture stress ( $\sim 13$ times) and fracture strain ( 2.6 times) in the direction parallel to shear flow than in the perpendicular direction. In this study, we extended this analysis of these mechanical properties towards Young's modulus and strain hardening. Since we believe that strain hardening is associated to the tendency to form fibrous morphology this should be further investigated. Strain hardening could provide information about the interactions between particles (in this case micelles) in a dense dispersion.

Microscale characteristics, such as the size distribution and the shape of air bubbles in calcium caseinate material have been studied with reflective light microscopy and X-ray tomography (XRT). Both methods revealed that air bubbles are slightly deformed in the shear flow direction, thus contributing to the structural anisotropy. However, XRT can only be used for phases that have sufficient contrast of density, while normal light microscopy does not give the 3D shape of phase domains. Confocal scanning laser microscopy can show 3D information to some degree, and can be used with phases that are similar in refractive index by using fluorescent markers, such as with protein and maltodextrin. Moreover, SEM images suggested that calcium caseinate material has structural anisotropy on a submicron length scale (100-200 nm). However, sample preparation with SEM is extensive, and it cannot be ruled out that the protein alignment observed from SEM could be induced during sample preparation. 
All microscopic methods, such as light and electron microscopy only give information about a small sample of a much bigger material. It is therefore nearly impossible to draw statistically significant conclusions from those images alone. This is somewhat better with XRT, even though the size of the samples here is still relatively small. For more reliable information we need to resort to analyses on larger scales. Recently, the structural anisotropy of calcium caseinate was studied using the Small Angle Neutron Scattering (SANS) technique (Tian, et al., to be submitted). No pre-treatment on the sample is needed for this technique. The anisotropic 2D scattering pattern indicated the existence of structural anisotropy at the submicron length scale. SANS analysis revealed a fibre diameter of $\sim 100 \mathrm{~nm}$, which is in line with SEM observations. This type of analysis could help to obtain more quantitative understanding of the structure itself, although the scattering profiles needs extensive interpretation and the assumptions made will also influence the outcome.

\subsection{Structure stability}

Food products need to be stored for distribution and convenient delivery to the consumers. Thus, proper storage conditions are necessary to maintain the fibrous structure. To assess this, sheared and enzymatically cross-linked calcium caseinate materials were stored at various storage temperatures $\left(20,4\right.$ and $\left.-20{ }^{\circ} \mathrm{C}\right)$ for $24 \mathrm{~h}$. The fresh material had a highly fibrous appearance when tearing it along the shear flow direction. The material stored at $-20{ }^{\circ} \mathrm{C}$ with subsequent thawing still showed a fibrous appearance that was comparable to that of the fresh sample. The material stored at $20^{\circ} \mathrm{C}$ or $4{ }^{\circ} \mathrm{C}$ had an altered structure and revealed a layered structure upon tearing after storage. The tensile properties were in line with these observations (Fig. 6-6). Compared with the fresh sample, the tensile properties of the samples stored at $20{ }^{\circ} \mathrm{C}$ increased greatly in both parallel and perpendicular direction to the shear flow, indicative of annealing of the structure. At the same time, it showed much lower anisotropy indices.

The conclusion is that the fibrous appearance remains for months when the material is kept frozen at low temperatures. The crystallization of the water results in a matrix that is close to its glass transition and thus will not evolve in time. At 4 and $20^{\circ} \mathrm{C}$, the material is still fully hydrated and deformable and can anneal its structure into a homogeneous mass. 

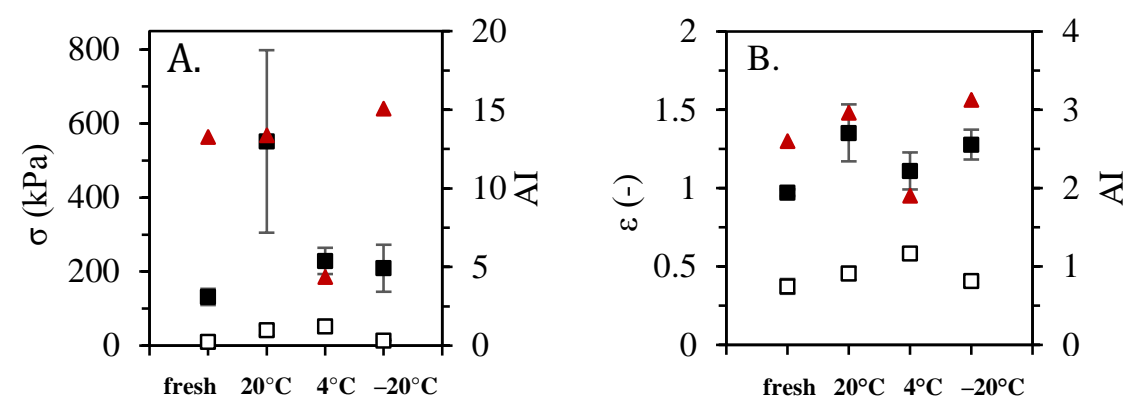

Fig. 6-6. Tensile properties: (A) fracture stress $\sigma$ and (B) fracture strain $\varepsilon$ of $30 \mathrm{wt} \%$ sheared and enzymatic cross-linked calcium caseinate materials after storing at different temperature for $24 \mathrm{~h}$. Fresh samples were measured as a reference. The error bars for the tensile properties are \pm standard deviation. The anisotropy index AI $(\boldsymbol{\Delta})$ is the ratio between the average values measured in the parallel (ם) and perpendicular $(\square)$ directions to the shear flow.

\subsection{Future perspective}

Plant-based analogues are important both for ensuring food security with a larger world population and for contributing to our health. With respect to a meat-like structure, the fibrous materials that can be achieved with calcium caseinate are highly relevant for this category of food products. So far, the use of plant proteins did not result in similar fibrousness. Hence it is relevant to consider whether the understanding of the structure formation obtained in this thesis, can be extrapolated to plant proteins.

Shear-induced structure formation from spray-dried calcium caseinate combines the benefits of self-assembly and forced assembly. It is not trivial to translate this towards other proteins, as different proteins have different properties. We can relate the behaviour of concentrated calcium caseinate dispersions to the behaviour of synthetic polymers under shear, which have been extensively studied. Spherical particles at micron length scale that dispersed in a viscoelastic fluid align during shearing; a phenomenon often indicated as shear banding. This alignment generally depends on shear thinning behaviour and the presence of significant normal stress differences (Jaensson, et al., 2016; Van Loon et al., 2014). Wall confinement, the volume fraction of the particle concentration, and the applied shear rate all influence this alignment (Pasquino et al., 2013; Van Loon et al., 2014). We believe that the alignment that we see in the caseinate dispersions is based on the same phenomenon. Calcium caseinate dispersions exhibit well-defined aggregates (micelles) sizes due to their self-assembly, and the mild association between the 
aggregates that is induced by the divalent $\mathrm{Ca}^{2+}$ give it sufficient non-Newtonian behaviour to induce the banding phenomenon, leading to string formation. This is in agreement with the fact that the structure formation is only found in concentrated dispersions; dispersions with much lower concentrations (below $20 \mathrm{wt} \%$ ) do not show this behaviour. Moreover, wall confinement might be also a very relevant parameter for promoting fibre formation. We found that a more pronounced fibrous structure was obtained when using an inner cone with a larger angle $\left(5^{\circ}\right)$, compared to the cone with an angle of $2.5^{\circ}$ used in this thesis (Fig. 1-1B).

It may be interesting to investigate plant proteins to form fibrous materials using the same structuring mechanism as calcium caseinate. Clearly, the plant protein dispersion should contain small aggregates of well-defined size $(100-300 \mathrm{~nm})$ with weak association, which are prone to alignment under flow. The weak association may be induced with a second phase (e.g., by depletion flocculation), or by adding a component that acts as a bridge, as $\mathrm{Ca}^{2+}$ does for caseinate. However, plant proteins are generally globular proteins that do not associate, at least not in a highly defined manner. The induction of associative behaviour is also more complex, since there is no natural interaction leading to such association. Therefore, the phase separation is generally imposed by combining two incompatible phases, such as a (plant) protein and a polysaccharide. This is similar to the use of the calcium caseinate-maltodextrin dispersions in this thesis (Chapter 5).

The structure from these dispersions showed similarities to the structure obtained with soy protein isolate (SPI)-pectin blends (Dekkers et al., 2018; Dekkers et al., 2016). The starting ingredients form a concentrated two-phase biopolymer blend. During shearing, the dispersed phase may coalesce, deform and break up. Furthermore, the addition of the polysaccharide to the protein results in $\sim 10 \%(\mathrm{v} / \mathrm{v})$ air holdup. These air bubbles were deformed and aligned in the shear flow direction. Also in these materials, it can be hypothesized that air bubbles are nuclei for rupture (stress concentrators) when such material is extended perpendicular the shear flow direction. The size and orientation of the weak, dispersed phase (polysaccharide and air) explain the different fracture properties when stretching the material parallel and perpendicular to the shear flow (Fig. 6-5). However, differences also were observed. In SPI-pectin blends, the addition of pectin leads to elongated, dispersed pectin droplets after shearing giving fibrous appearance and mechanical anisotropy upon tearing. In caseinate-maltodextrin dispersions, there was no 
orientation of the maltodextrin phase, even though macroscopic anisotropy could be achieved.

The structure formation in caseinate-maltodextrin dispersions indicated that only deformed air bubbles in the matrix are able to create microscopic anisotropy. The effect of the polysaccharide phase is more likely to introduce and stabilise air bubbles upon shear deformation. This mechanism could be developed for other plant proteins as well. First, it is important to obtain a biopolymer blend in which air bubbles are stabilised, which could be achieved by adding polysaccharides or gluten as a dispersed phase into a protein phase. Polysaccharides (e.g. carrageenan or maltodextrin) are often added as stabilizing agent in foams. These components do not only increase the viscosity but also create an elastic network (Makri and Doxastakis, 2006; Pycia, et al., 2017; Pycia, et al., 2016; Smith, et al., 2000). Gluten, which is often added to SPI for making a range of fibrous structures, is also important for hindering coalescence of bubbles during bread making due to strain hardening behaviour (Dobraszczyk et al., 2003; Kokelaar et al., 1996; Vliet et al., 1992). In addition, the ratio of volume fractions between the protein and the additional phase should be large to prevent phase inversion upon shear deformation, an effect that is detrimental for formation of fibrous materials.

Besides choosing the right dispersed phase, the temperature at which the blend is sheared, is as well important for the air holdup. The fibre formation in the caseinate-maltodextrin dispersion revealed that very high temperature is not necessary for the formation of a fibrous material. By applying relatively low temperature, it is possible to minimise heating-induced changes in the blend, which probably impacts the viscosity and deformability over time. However, very low temperature may require a high torque to deform the blend. Thus, the temperature should be adjusted based on the properties of the biopolymer phases. Last but not least, shear rate and processing time determine the extent of deformation of air bubbles, which also need to be optimised. 


\section{Appendix 6A}
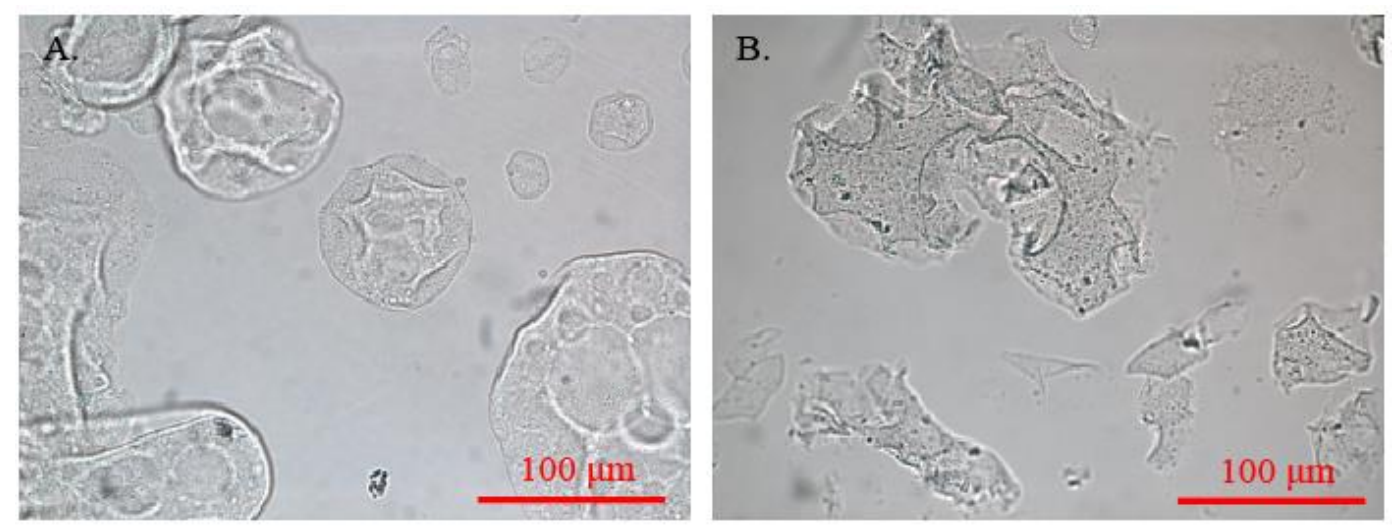

Fig 6A-1. Morphology of spray-dried (A) and roller-dried (B) calcium caseinate in the sediment (v. Fig. 6-3). $10 \mathrm{wt} \%$ dispersions were mixed for $5 \mathrm{~min}$, and then centrifuged at $18,400 \mathrm{~g}$ for $5 \mathrm{~min}$.

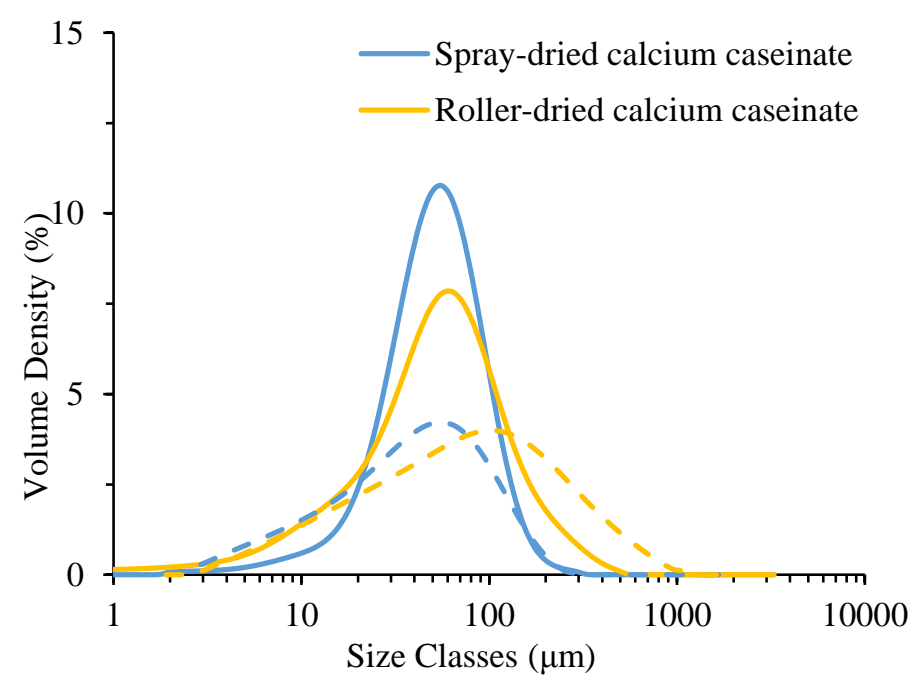

Fig 6A-2. Size distribution of spray-dried and roller dried calcium caseinate powder, and their sediments (v. Fig. 6-3). 
References 
Akkermans, C., van der Goot, A.J., Venema, P., van der Linden, E., Boom, R.M., 2008a. Formation of fibrillar whey protein aggregates: Influence of heat and shear treatment, and resulting rheology. Food Hydrocoll. 22, 1315-1325.

Akkermans, C., Van der Goot, A.J., Venema, P., Van der Linden, E., Boom, R.M., 2008. Properties of protein fibrils in whey protein isolate solutions: Microstructure, flow behaviour and gelation. Int. Dairy J. 18, 1034-1042.

Akkermans, C., Venema, P., van der Goot, A.J., Gruppen, H., Bakx, E.J., Boom, R.M., van der Linden, E., 2008b. Peptides are building blocks of heat-induced fibrillar protein aggregates of $\beta$-lactoglobulin formed at pH 2. Biomacromolecules 9, 1474-1479.

Allen, K.E., Murray, B.S., Dickinson, E., 2008. Development of a model whipped cream: Effects of emulsion droplet liquid/solid character and added hydrocolloid. Food Hydrocoll. $22,690-699$.

Andersons, J., Kirpluks, M., Stiebra, L., Cabulis, U., 2015. The effect of a circular hole on the tensile strength of neat and filled rigid PUR foams. Theor. Appl. Fract. Mech. 78, 8-14.

Arêas, J.A.G., 1992. Extrusion of food proteins. Crit. Rev. Food Sci. Nutr. 32, 365-392.

Augustin, M.A., Oliver, C.M., Hemar, Y., 2011. Casein, caseinates, and milk protein concentrates. Dairy Ingredients Food Process. 161-178.

Bast, R., Sharma, P., Easton, H.K.B., Dessev, T.T., Lad, M., Munro, P.A., 2015. Tensile testing to quantitate the anisotropy and strain hardening of mozzarella cheese. Int. Dairy J. 44, 614.

Bastier, P., Dumay, E., Cheftel, J.C., 1993. Physico-chemical and functional properties of commercial caseinates. LWT - Food Sci. Technol. 26, 529-537.

Bhaskaracharya, R.K., Shah, N.P., 2001. Texture and microstructure of skim milk mozzarella cheeses made using fat replacers. Aust. J. Dairy Technol. 56 (1), 9-14.

Bolder, S.G., Hendrickx, H., Sagis, L.M.C., Van Der Linden, E., 2006. Fibril assemblies in aqueous whey protein mixtures. J. Agric. Food Chem. 54, 4229-4234. 
Boom, R.M., 2011. Nanotechnology in food production, in: Nanotechnology in the AgriFood Sector: Implications for the Future. pp. 39-56.

Campbell, G.M., Mougeot, E., 1999. Creation and characterisation of aerated food products. Trends Food Sci. Technol. 10, 283-296.

Carr, A., Golding, M., 2016. Functional milk proteins production and utilization: caseinbased ingredients, in: McSweeney, P.L.H., O’Mahony, J.A. (Eds.), Advanced Dairy Chemistry: Volume 1B: Proteins: Applied Aspects. Springer, New York, NY, pp. 35-66.

Carr, A.J., Southward, C.R., Creamer, L.K., 2003. Protein hydration and viscosity of dairy fluids, in: Fox, P.F., McSweeney, P.L.H. (Eds.), Advanced Dairy Chemistry-1 Proteins. Springer, New York, NY, pp. 1289-1323.

Chandan, R.C., Kilara, A., 2011. Dairy Ingredients for Food Processing. Blackwell, Ames, IA.

Cheftel, J.C., Kitagawa, M., Queguiner, C., 1992. New Protein Texturization Processes by Extrusion Cooking at High Moisture Levels, Food Reviews International. 8, 235-275.

Chen, X., Wu, S., Zhou, J., 2013. Influence of porosity on compressive and tensile strength of cement mortar. Constr. Build. Mater. 40, 869-874.

Cheng, J., Carreau, P.J., 1994. Aerated mixing of viscoelastic fluids with helical ribbons impellers. Chem. Eng. Sci. 49, 1965-1972.

Chin, N.L., Martin, P.J., Campbell, G.M., 2005. Dough aeration and rheology: Part 3. Effect of the presence of gas bubbles in bread dough on measured bulk rheology and work input rate. J. Sci. Food Agric. 85, 2203-2212.

Cho, K.Y., Rizvi, S.S.H., 2009. 3D Microstructure of supercritical fluid extrudates. II: Cell anisotropy and the mechanical properties. Food Res. Int. 42, 603-611.

Dalgleigh, D.G., Law, A.J.R., 1988. Sodium caseinates - composition and properties of different preparations. Int. J. Dairy Technol. 41, 1-4.

de Belder, A.N., Granath, K., 1973. Preparation and properties of fluorescein-labelled dextrans. Carbohydr. Res. 30, 375-378. 
De Kruif, C.G., Holt, C., 2003. Casein Micelle Structure, Functions and Interactions, in: Advanced Dairy Chemistry-1 Proteins. Springer, New York, pp. 233-276.

De Kruif, C.G., Huppertz, T., Urban, V.S., Petukhov, A. V., 2012. Casein micelles and their internal structure. Adv. Colloid Interface Sci. 171-172, 36-52.

Dekkers, B.L., Emin, M.A., Boom, R.M., van der Goot, A.J., 2018. The phase properties of soy protein and wheat gluten in a blend for fibrous structure formation. Food Hydrocoll. 79, 273-281.

Dekkers, B.L., Hamoen, R., Boom, R.M., van der Goot, A.J., 2018. Understanding fiber formation in a concentrated SPI-pectin blend. J. Food Eng. 222, 1-20.

Dekkers, B.L., Nikiforidis, C. V, van der Goot, A.J., 2016. Shear-induced fibrous structure formation from a pectin/SPI blend. Innov. Food Sci. Emerg. Technol. 36, 193-200.

Dissanayake, M., Liyanaarachchi, S., Vasiljevic, T., 2012. Functional properties of whey proteins microparticulated at low pH. J. Dairy Sci. 95, 1667-1679.

Dobraszczyk, B.J., Smewing, J., Albertini, M., Maesmans, G., Schofield, J.D., 2003. Extensional rheology and stability of gas cell walls in bread doughs at elevated temperatures in relation to breadmaking performance. Cereal Chem. 80, 218-224.

Ferry, J.D., 1980. Viscoelastic properties of polymer solutions, 3rd ed. ed. Wiley, New York. Fox, P.F., Mulvihill, D.M., 1982. Milk proteins: molecular, colloidal and functional properties. J. Dairy Res. 49, 679-693.

Gibson, L. J., Ashby, M.F., 1997. Cellular solids: structure and properties. 2nd ed. Cambridge Cambridge Univ. Press.

Gibson, L.J., 2012. The hierarchical structure and mechanics of plant materials. J. R. Soc. Interface 9, 2749-2766.

Grabowska, K.J., Tekidou, S., Boom, R.M., van der Goot, A.-J., 2014. Shear structuring as a new method to make anisotropic structures from soy-gluten blends. Food Res. Int. 64, 743-751. 
Grabowska, K.J., van der Goot, A.J., Boom, R.M., 2012. Salt-modulated structure formation in a dense calcium caseinate system. Food Hydrocoll. 29, 42-47.

Grabowska, K.J., Zhu, S., Dekkers, B.L., de Ruijter, N.C.A., Gieteling, J., van der Goot, A.J., 2016. Shear-induced structuring as a tool to make anisotropic materials using soy protein concentrate. J. Food Eng. 188, 77-86.

Greil, P., Lifka, T., Kaindl, A., 1998. Biomorphic cellular silicon carbide ceramics from wood: II. Mechanical properties. J. Eur. Ceram. Soc. 18, 1975-1983.

Greil, P., Vogli, E., Fey, T., Bezold, A., Popovska, N., Gerhard, H., Sieber, H., 2002. Effect of microstucture on the fracture behavior of biomorphous silicon carbide ceramics. J. Eur. Ceram. Soc. 22, 2697-2707.

Griffiths, L., Heap, M.J., Xu, T., Chen, C., Baud, P., 2017. The influence of pore geometry and orientation on the strength and stiffness of porous rock. J. Struct. Geol. 96, 149-160.

Habibi, M.K., Lu, Y., 2014. Crack propagation in bamboo's hierarchical cellular structure. Sci. Rep. 4, 1-6.

HadjSadok, A., Pitkowski, A., Nicolai, T., Benyahia, L., Moulai-Mostefa, N., 2008. Characterisation of sodium caseinate as a function of ionic strength, $\mathrm{pH}$ and temperature using static and dynamic light scattering. Food Hydrocoll. 22, 1460-1466.

Haghpanah, B., Oftadeh, R., Papadopoulos, J., Vaziri, A., 2013. Self-similar hierarchical honeycombs. Proc. R. Soc. A.

Hoek, A.C., Luning, P.A., Weijzen, P., Engels, W., Kok, F.J., de Graaf, C., 2011. Replacement of meat by meat substitutes. A survey on person- and product-related factors in consumer acceptance. Appetite 56, 662-673.

Hogan, S.A., Chaurin, V., O’Kennedy, B.T., Kelly, P.M., 2012. Influence of dairy proteins on textural changes in high-protein bars. Int. Dairy J. 26, 58-65.

Huppertz, T., Fox, P.F., Kelly, A.L., 2017. The caseins: Structure, stability, and functionality, Proteins in Food Processing: Second Edition. Woodhead Publishing, Duxford, UK. 
Hwang, W.R., Hulsen, M.A., 2011. Structure formation of non-colloidal particles in viscoelastic fluids subjected to simple shear flow. Macromol. Mater. Eng. 296, 321-330.

Hyun, S.K., Murakami, K., Nakajima, H., 2001. Anisotropic mechanical properties of porous copper fabricated by unidirectional solidification. Mater. Sci. Eng. A 299, 241-248.

Jack, M., 2016. Cheese Analogues. J. Food Dairy Technol. 4, 44-48.

Jaensson, N.O., Hulsen, M.A., Anderson, P.D., 2016. Direct numerical simulation of particle alignment in viscoelastic fluids. J. Nonnewton. Fluid Mech. 235, 125-142.

Ji, S., Gu, Q., Xia, B., 2006. Porosity dependence of mechanical properties of solid materials. J. Mater. Sci. 41, 1757-1768.

Kalab, M., Emmons, D.., 1974. Milk gel structure III . Microstructure of skim milk powder and gels as related to the drying procedure. Milchwissenschaft 29, 585-589.

Kokelaar, J.J., van Vliet, T., Prins, A., 1996. Strain Hardening Properties and Extensibility of Flour and Gluten Doughs in Relation to Breadmaking Performance. J. Cereal Sci. 24, 199214.

Kurtz, S.M., Pruitt, L.A., Jewett, C.W., Foulds, J.R., Edidin, A.A., 1999. Radiation and chemical crosslinking promote strain hardening behavior and molecular alignment in ultra-high molecular weight polyethylene during multi-axial loading conditions. Biomaterials 20, 1449-1462.

Lakes, R., 1993. Materials with structural hierarchy. Nature 361, 511-515.

Lepetit, J., Culioli, J., 1994. Mechanical properties of meat. Meat Sci. 36, 203-237.

Lin, S., Huff, H.E., Hsieh, F., 2000. Texture and Chemical Characteristics of Soy Protein Meat Analog Extruded at High Moisture. J. Food Sci. 65, 264-269.

Loveday, S.M., Rao, M.A., Creamer, L.K., Singh, H., 2010. Rheological behavior of highconcentration sodium caseinate dispersions. J. Food Sci. 75, 30-35.

Lucey, J.A., Horne, D.S., 2018. Perspectives on casein interactions. Int. Dairy J. 85, 56-65. 
Makri, E.A., Doxastakis, G.I., 2006. Surface tension of Phaseolus vulgaris and coccineus proteins and effect of polysaccharides on their foaming properties. Food Chem. 101, 3748.

Manoj, P., Kasapis, S., Chronakis, I.S., 1996. Gelation and phase separation in maltodextrincaseinate systems. Food Hydrocoll. 10, 407-420.

Manski, J.M., van der Goot, A.J., Boom, R.M., 2007a. Formation of fibrous materials from dense calcium caseinate dispersions. Biomacromolecules 8, 1271-1279.

Manski, J.M., van der Goot, A.J., Boom, R.M., 2007b. Advances in structure formation of anisotropic protein-rich foods through novel processing concepts. Trends Food Sci. Technol. 18, 546-557.

Manski, J.M., van der Zalm, E.E.J., van der Goot, A.J., Boom, R.M., 2008. Influence of process parameters on formation of fibrous materials from dense calcium caseinate dispersions and fat. Food Hydrocoll. 22, 587-600.

Manski, J.M., van Riemsdijk, L.E., Boom, R.M., van der Goot, A.J., 2007c. Importance of intrinsic properties of dense caseinate dispersions for structure formation. Biomacromolecules 8, 3540-3547.

Masmoudi, M., Kaddouri, W., Kanit, T., Madani, S., Ramtani, S., Imad, A., 2017. Modeling of the effect of the void shape on effective ultimate tensile strength of porous materials: Numerical homogenization versus experimental results. Int. J. Mech. Sci. 130, 497-507.

McMahon, D.J., Alleyne, M.C., Fife, R.L., Oberg, C.J., 1996. Use of Fat Replacers in Low Fat Mozzarella Cheese. J. Dairy Sci. 79, 1911-1921.

Mioche, L., Bourdiol, P., Monier, S., 2003. Chewing behaviour and bolus formation during mastication of meat with different textures. Arch. Oral Biol. 48, 193-200.

Mioche, L., Hiiemae, K.M., Palmer, J.B., 2002. A postero-anterior videofluorographic study of the intra-oral management of food in man. Arch. Oral Biol. 47, 267-280.

Morales, F.J., Van Boekel, M.A.J.S., 1998. A study on advanced Maillard reaction in heated casein/sugar solutions: Colour formation. Int. Dairy J. 8, 907-915. 
Moughal, K.I., Munro, P.A., Singh, H., 2000. Suspension stability and size distribution of particles in reconstituted, commercial calcium caseinates. Int. Dairy J. 10, 683-690.

Mulvihill, D.M., Ennis, M.P., 2003. Functional milk proteins: production and utilization, in: Fox, P.F., McSweeney, P.L.H. (Eds.), Advanced Dairy Chemistry- Proteins. Springer, New York, NY, pp. 1175-1228.

Nagashima, K., Mitsumata, T., 2011. Isotropy And Anisotropy In Agricul. Encycl. Agrophysics 407-409.

Nakajima, H., 2007. Fabrication, properties and application of porous metals with directional pores. Prog. Mater. Sci. 52, 1091-1173.

O’Regan, J., Mulvihill, D.M., 2011. Milk protein products | Caseins and caseinates, industrial production, compositional standards, specifications, and regulatory aspects. Encycl. Dairy Sci.

Oberg, E.N., Oberg, C.J., Motawee, M.M., Martini, S., McMahon, D.J., 2015. Increasing stringiness of low-fat mozzarella string cheese using polysaccharides. J. Dairy Sci. 98, 4243-4254.

Olurin, O.B., Fleck, N.A., Ashby, M.F., 2001. Tensile and compressive failure of notched cellular foams. Adv. Eng. Mater. 3, 55-58.

Ono, T., Yoshida, M., Tanaami, H., Ohkosi, H., 1999. Changes in casein micelle size induced by heating. Int. Dairy J. 9, 405-406.

Pasquino, R., Panariello, D., Grizzuti, N., 2013. Migration and alignment of spherical particles in sheared viscoelastic suspensions. A quantitative determination of the flowinduced self-assembly kinetics. J. Colloid Interface Sci. 394, 49-54.

Peighambardoust, S.H., Hamer, R.J., Boom, R.M., van der Goot, A.J., 2008. Migration of gluten under shear flow as a novel mechanism for separating wheat flour into gluten and starch. J. Cereal Sci. 48, 327-338. 
Peighambardoust, S.H., Van der Goot, A.J., Van Vliet, T., Hamer, R.J., Boom, R.M., 2006. Microstructure formation and rheological behaviour of dough under simple shear flow. J. Cereal Sci. 43, 183-197.

Peressini, D., Peighambardoust, S.H., Hamer, R.J., Sensidoni, A., Van Der Goot, A.J., 2008. Effect of shear rate on microstructure and rheological properties of sheared wheat doughs. J. Cereal Sci. 48, 426-438.

Peters, J.P.C.M., Luyten, H., Alting, A.C., Boom, R.M., van der Goot, A.J., 2015. Effect of crosslink density on the water-binding capacity of whey protein microparticles. Food Hydrocoll. 44, 277-284.

Purwanti, N., Moerkens, A., van der Goot, A.J., Boom, R., 2012. Reducing the stiffness of concentrated whey protein isolate (WPI) gels by using WPI microparticles. Food Hydrocoll. 26, 240-248.

Purwanti, N., Peters, J.P.C.M., Van Der Goot, A.J., n.d. Protein micro-structuring as a tool to texturize protein foods.

Pycia, K., Gałkowska, D., Juszczak, L., 2017. Maltodextrins produced from chemically modified starches as agents affecting stability and rheological properties of albumin foam. LWT 80, 394-400.

Pycia, K., Juszczak, L., Gałkowska, D., 2016. Effect of native potato starch maltodextrins on stability and rheological properties of albumin foams. Starch - Stärke 68, 611-620.

Quo, M., Fox, P.F., Mahammad, K.S., Flynn, A., 1989. Heat-induced changes in sodium caseinate. J. Dairy Res. 56, 503-512.

Rice, R.W., 1996. The porosity dependence of physical properties of materials: a summary review. Key Eng. Mater. 115, 1-20.

Rice, R.W., 1996. Evaluation and extension of physical property-porosity models based on minimum solid area. J. Mater. Sci. 31, 102-118.

Rice, R.W., 1993. Comparison of stress concentration versus minimum solid area based mechanical property-porosity relations. J. Mater. Sci. 28, 2187-2190. 
Rolls, B.A., Porter, J.W.G., 1973. Some effects of processing and storage on the nutritive value of milk and milk products. Proc. Nutr. Soc. 32, 9-15.

Rondanelli, M., Klersy, C., Terracol, G., Talluri, J., Maugeri, R., Guido, D., Faliva, M.A., Solerte, B.S., Fioravanti, M., Lukaski, H., Perna, S., 2016. Whey protein, amino acids, and Vitamin D supplementation with physical activity increases fat-free mass and strength, functionality, and quality of life and decreases inflammation in sarcopenic elderly. Am. J. Clin. Nutr. 103, 830-840.

Ross-Murphy, S.B., 1995. Structure-property relationships in food biopolymer gels and solutions. Cit. J. Rheol. 39, 1451.

Ross, K.A., Pyrak-Nolte, L.J., Campanella, O.H., 2006. The effect of mixing conditions on the material properties of an agar gel - Microstructural and macrostructural considerations. Food Hydrocoll. 20, 79-87.

Sağlam, D., Venema, P., de Vries, R., van den Berg, M., van der Linden, E., 2014. Whey protein particles modulate mechanical properties of gels at high protein concentrations. Food Hydrocoll. 38, 163-171.

Schuck, P., 2011. Milk powder: physical and functional properties of milk powders. Encycl. Dairy Sci.

Shang, N., Chaplot, S., Wu, J., 2018. Food proteins for health and nutrition. Proteins Food Process. 301-336.

Shurtleff, W., Aoyagi, A., 2016. History of modern soy protein ingredients-isolates, concentrates, and textured soy protein products (1911-2016): Extensively annotated bibliography and sourcebook.

Smith, A.K., Goff, H.D., Kakuda, Y., 2000. Microstructure and rheological properties of whipped cream as affected by heat treatment and addition of stabilizer. Int. Dairy J. 10, 295-301.

Stanzl-Tschegg, S.E., Keunecke, D., Tschegg, E.K., 2011. Fracture tolerance of reaction wood (yew and spruce wood in the TR crack propagation system). J. Mech. Behav. Biomed. Mater. 4, 688-698. 
Thomar, P., Benyahia, L., Durand, D., Nicolai, T., 2014. The influence of adding monovalent salt on the rheology of concentrated sodium caseinate suspensions and the solubility of calcium caseinate. Int. Dairy J. 37, 48-54.

Thomar, P., Durand, D., Benyahia, L., Nicolai, T., 2012. Slow dynamics and structure in jammed milk protein suspensions. Faraday Discuss. 158, 325-339.

Thomar, P., Nicolai, T., Benyahia, L., Durand, D., 2013. Comparative study of the rheology and the structure of sodium and calcium caseinate solutions. Int. Dairy J. 31, 100-106.

Tian, B., Wang, Z., van der Goot, A.J., Bouwman, W.G., 2018. Air bubbles in fibrous caseinate gels investigated by neutron refraction, X-ray tomography and refractive microscope. Food Hydrocoll. 83, 287-295.

Tian, B., Wang, Z., van der Goot, A.J., Bouwman, W.G., 2019. Anisotropy and structure of the calcium caseinate fibres studied by neutron scattering. Food Hydrocoll. To be submitted.

Tiwari, S., Bhattacharya, S., 2011a. Aeration of model gels: rheological characteristics of gellan and agar gels. J. Food Eng. 107, 134-139.

Tiwari, S., Bhattacharya, S., 2011b. Aeration of model gels: rheological characteristics of gellan and agar gels. J. Food Eng. 107, 134-139.

Tolstoguzov, V., 2006. Texturising by phase separation. Biotechnol. Adv. 24, 626-628.

Torres, I.C., Janhøj, T., Mikkelsen, B.Ø., Ipsen, R., 2011. Effect of microparticulated whey protein with varying content of denatured protein on the rheological and sensory characteristics of low-fat yoghurt. Int. Dairy J. 21, 645-655.

van den Einde, R.M., Bolsius, A., van Soest, J.J.G., Janssen, L.P.B.M., van der Goot, A.J., Boom, R.M., 2004. The effect of thermomechanical treatment on starch breakdown and the consequences for process design. Carbohydr. Polym. 55, 57-63.

van den Einde, R.M., van der Veen, M.E., Bosman, H., van der Goot, A.J., Boom, R.M., 2005. Modeling macromolecular degradation of corn starch in a twin screw extruder. J. Food Eng. 66, 147-154. 
van der Zalm, E.E.J., Berghout, J.A.M., van der Goot, A.J., Boom, R.M., 2012. Starch-gluten separation by shearing: Influence of the device geometry. Chem. Eng. Sci. 73, 421-430.

Van Loon, S., Fransaer, J., Clasen, C., Vermant, J., 2014. String formation in sheared suspensions in rheologically complex media: The essential role of shear thinning. J. Rheol. $58,237-254$.

van Riemsdijk, L.E., Pelgrom, P.J.M., van der Goot, A.J., Boom, R.M., Hamer, R.J., 2011. A novel method to prepare gluten-free dough using a meso-structured whey protein particle system. J. Cereal Sci. 53, 133-138.

Veerman, C., Ruis, H., Sagis, L.M.C., van der Linden, E., 2002. Effect of electrostatic interactions on the percolation concentration of fibrillar $\beta$-lactoglobulin gels. Biomacromolecules 3, 869-873.

Vincent, J.F. V, 2011. Unusual uses of holes-With input from biology. J. Mech. Behav. Biomed. Mater. 4, 682-687.

Vliet, T. van, Janssen, A.M., Bloksma, A.H., Walstra, P., 1992. Strain hardening of dough as a requirement for gas retention. J. Texture Stud. 23, 439-460.

Wang, S., Yang, L., Lu, J., Mu, Y., 2014. High-protein breakfast promotes weight loss by suppressing subsequent food intake and regulating appetite hormones in obese Chinese adolescents. Hormone Research in Paediatrics. 83, 19-25.

Wang, Z., Tian, B., Boom, R., van der Goot, A.J., 2019a. Air bubbles in calcium caseinate fibrous material enhances anisotropy. Food Hydrocoll. 87, 497-505.

Wang, Z., Tian, B., Boom, R., van der Goot, A.J., 2019b. Understanding the role of air and protein phase on mechanical anisotropy of calcium caseinate fibers. Food Res. Int. In Press.

Wegst, U.G.K., Bai, H., Saiz, E., Tomsia, A.P., Ritchie, R.O., 2015. Bioinspired structural materials. Nat. Mater. 14, 23-36.

Won, D., Kim, C., 2004. Alignment and aggregation of spherical particles in viscoelastic fluid under shear flow. J. Nonnewton. Fluid Mech. 117, 141-146. 
Yang, S.W., Watkinson, P., Gillies, G., James, B.J., 2016. Microstructural transformations in anisotropy and melt-stretch properties of low moisture part skim mozzarella cheese. Int. Dairy J. 62, 19-27.

Yokoyama, K., Ohtsuka, T., Kuraishi, C., Ono, K., Kita, Y., Arakawa, T., Ejima, D., 2003. Gelation of food protein induced by recombinant microbial transglutaminase. J. Food Sci. $68,48-51$.

Yuryev, V.P., Zasypkin, D. V., Ghenin, Y. V., Zhukov, V.A., Alexeyev, V. V., Tolstoguzov, V.B., 1991. Role of maltodextrin in promoting structure formation in extruded soya isolate. Carbohydr. Polym. 15, 243-253.

Zúñiga, R.N., Aguilera, J.M., 2009. Structure-fracture relationships in gas-filled gelatin gels. Food Hydrocoll. 23, 1351-1357. 
Summary 
The development of new generation meat analogues contributes to a more sustainable food production. The creation of fibrous structures within these kind of protein foods is of relevance. Concentrated calcium caseinate dispersions can be transformed into hierarchically fibrous materials using shear deformation. The fibrousness of these materials is more pronounced compared with materials created from plant proteins. Thus, calcium caseinate can be used as a model system for studying fibrous structure formation.

In previous studies, the transformation of calcium caseinate into distinct fibrous structures was attributed to the caseinate micelles with attractive interactions between them. However, the mechanism that was previously described cannot fully explain all the phenomena in structure formation, i.e. the large differences between batches of caseinate. Therefore, it was deemed essential to further elucidate the mechanism of fibre formation in concentrated calcium caseinate dispersion.

In Chapter 2, the role of air in calcium caseinate was investigated by studying the structure and mechanical properties of the final material. Although anisotropy was present in deaerated calcium caseinate dispersions after shearing, air incorporation enhanced the fibrous appearance and mechanical anisotropy of the sheared calcium caseinate materials. Air bubbles were therefore considered as an additional, important phase, causing the material to become tougher with strain hardening in the direction parallel to the fibres, and making the material more susceptible to fracture upon stretching in the perpendicular direction.

Chapter 3 further describes the relative importance of air bubbles in the protein matrix on the mechanical anisotropy of calcium caseinate material. In this study, the effect of air on mechanical anisotropy of these fibrous materials was described with a load-bearing model, with the void fraction, and the bubble length and width as input parameters. The anisotropy of the protein phase was estimated using materials obtained from deaerated dispersions after shearing at different shear rates. It was concluded that the deformation of air bubbles could only partly explain the mechanical anisotropy; the anisotropy of the protein phase is more important. Based on all results, we further hypothesised that the anisotropy of the protein phase was affected by the air bubbles present during the structuring process. This effect was explained by a locally higher shear rate in the protein matrix during the structuring process. 
All previous studies on shear-induced calcium caseinate fibre formation were done using spray-dried powder. However, large differences were observed in the structuring potential and physical properties of spray-dried calcium caseinate (Scaca) and rollerdried calcium caseinate (Rcaca). In Chapter 4, it is described that Scaca formed more pronounced fibrous materials upon shearing than Rcaca. The rheological measurements revealed that the Scaca dispersion exhibited more solid-like behaviour. Besides, the particle size in excess water was larger for Rcaca $(2-300 \mu \mathrm{m})$, while Scaca mainly contained small caseinate aggregates $(<800 \mathrm{~nm}$ ). Finally, Rcaca was less susceptible to enzymatic crosslinking with transglutaminase. The differences in structuring potential and physical properties were explained by changes in the protein induced by the intensive thermal treatment during roller drying. The application of a similar thermal process to Scaca resulted in similar properties as Rcaca indeed. Clearly, the process history of calcium caseinate has major consequences for its fibre formation potential.

Chapter 5 describes another mechanism to create fibrous materials using roller-dried calcium caseinate powder. The method is based on the use of an extra phase in the initial dispersion, being maltodextrin. The addition of maltodextrin in a continuous protein phase led to the formation of fibrous materials and enhanced mechanical anisotropy after shearing. The maltodextrin addition resulted in a more solid-like dispersion and more air bubbles were entrapped in the sheared material. These air bubbles were large and elongated, which is of importance for the fibrous appearance on macroscale. Here, we found that the fibrous structure or mechanical anisotropy was a result of anisotropy on a mesoscale. No protein alignment was observed at a smaller length scale, which is in contrast to structured spray-dried calcium caseinate.

Chapter 6 reviews all results presented in this thesis and provides an overview of anisotropic structure formation principles with calcium caseinate. Further, different analytical techniques are discussed for studying the hierarchically fibrous structure. Finally, the insights on structuring of caseinate system were extrapolated towards other proteins. In conclusion, the results described in this thesis highlight the importance of air bubbles and contribute to a better understanding of the fibrous structure formation process based on shear cell technology. 


\section{Acknowledgement}

I would like to acknowledge all who helped my work presented in this thesis. Firstly, I would like to express my gratitude to my promotors. Dear Atze Jan, thank you for offering me the opportunity to do a PhD under your supervision. You always helped me to come back on track when my thoughts went too fast. I will never forget the first day that you picked me up in Schiphol Airport at 4 am. Dear Remko, thank you for your endless scientific thoughts. Your smart ideas always gave me light when I faced scientific challenges.

Secondly, I would like to thank Jarno, Jos and Maurice for the technical support and Martin for the help with financial matters. Marjan and Ilona, thank you so much for the excellent administrative works and sweets. I would like to express my appreciation to my students Laura, Astrid, Madelon, Saffiera, Yovita and Eline. Thank you so much for your valuable contributions to this thesis. It was a pleasure working with all of you. Bei and Birgit, it is a pleasure to collaborate with you. Thank you very much!

Thank you all my great officemates, Pachy, Kacie, Juliana, Pina, Anja, Jorien, Zulhaj, Birgit, Anna and Ivanna. It is great to have you around. Thank you so much for discussing researches, organizing the fantastic travels, sharing different culture and having great office dinners. Kacie, Angelica and Daniel, thank you for visiting me in China. Bijoy, Yu, Elvira, Dimitri and Jun, thank you for supporting me during thesis writing. Andrea, Konstantina and Sirinan, thank you for sharing the authentic and delicious food. Eline, it is great to have the defence on the same day with you. Thank all my fellow gardener, Jarno, Durk (and Juliana), Lu, Elvira, Wanqing and Sirinan. I really enjoyed the time with you in our beautiful garden. Birgit and Lu, thank you very much for being my paranymphs. I would like to express my appreciation to Jaap, Jan-Eise, Evelien, Victor, Qinhui, Emma, Floor, Steven, Jan and all other FPE colleagues. I really enjoyed all the scientific and playful activities that we did together: coffee breaks, We-days, labuitijes, borrels, groupdays, Leeuwarden weekends and PhD trips.

I would also like to thank all my Chinese friends in Wageningen: Gou Fang, Li Chunjie, Li Guohua, Zou Yi, Wu Jia, Ouyang Wenjing, Tan Meixiu, Li Huayi, Zhang Hao, Xue Wei, Zhang 
Peiyu, Zhang Ningyi, Xu Wei, Tang Kailei, Zhang Chunyue, Fahui Liu, Wu Qimeng, Kan Lijiao, Wang Zhijun, Zeng Zhe, Simon, Jiang Yibo and many others. Gou Fang, I am very lucky having you during my first two years in Wageningen as my best friend and neighbour. Thank you for introducing me to your friends, helping me quickly adapt to the new environment. Thank you for all the delicious food when I travelled back home. Thank you for sharing your passion and knowledge on plants. Many thanks to my housemates Luo Qi and Bian Mengxi. Thank you for all the delicious food, nice music and talks.

最后, 感谢我爱的家人们。亲爱的爷爷奶奶, 感谢你们的培养和教育, 没有你们我就 没有今天的成就。亲爱的爸爸, 妈妈, 叔叔, 婶婶, 大叔叔, 盛姨, 妹妹, 弟弟, 谢 谢你们对我在外求学的支持和鼓励。 


\section{About the author}

Zhaojun Wang (Fiona) was born in Hunan, China, on April 25 th 1990. In 2007, she started her bachelor's study of Food Science and Technology at Changsha University of Science and Technology in China. After graduating in 2011, she continued to study for a Master's degree at State Key Laboratory of Food Science and Technology at Nanchang University. She completed her Master thesis entitled 'Identification of volatile compounds

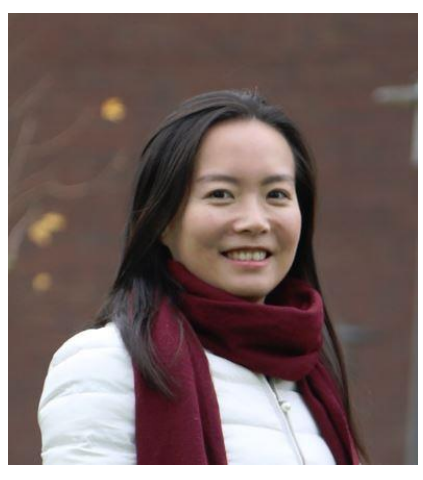
and organic acid from Choerospondias axillaris fruit'. In March 2015, she received scholarship from China Scholarship council to perform a PhD project at the Food Process Engineering group of Wageningen University \& Research. The result of her PhD research are presented in this thesis.

Contact e-mail: fionawang679@gmail.com 


\section{List of publications}

\section{This thesis:}

Wang, Z., Tian, B., Boom, R., \& van der Goot, A. J. (2019). Air bubbles in calcium caseinate fibrous material enhances anisotropy. Food Hydrocolloids, 87, 497-505.

Wang, Z., Tian, B., Boom, R., \& van der Goot, A. J. (2019). Understanding the role of air and protein phase on mechanical anisotropy of calcium caseinate fibres. Food Research International, 121, 862-869.

Wang, Z., Dekkers, B.L., Boom, R., \& van der Goot, A. J. (2019). Maltodextrin promotes calcium caseinate fibre formation through air inclusion. Food Hydrocolloids, 95, 143-151.

Wang, Z., Dekkers, B.L. \& van der Goot, A. J. (2019). Maltodextrin promotes calcium caseinate fibre formation through air inclusion. Journal of Food Engineering, under review.

\section{Other publication:}

Tian, B., Wang, Z., van der Goot, A. J., \& Bouwman, W. G. (2018). Air bubbles in fibrous caseinate gels investigated by neutron refraction, X-ray tomography and refractive microscope. Food Hydrocolloids, 83, 287-295. 


\section{Overview of completed training activities}

\section{Discipline specific activities}

\section{Courses}

Sustainability Analysis in Food and Biobased Production (VLAG, NL) 2015

The 15th European School on Rheology (Leuven University, BE) 2015

Food Proteins: Significance, Reactions and Modifications 2016

(VLAG/Copenhagen University, DK)

Process Economics and Cost Engineering (OSPT, NL) 2016

Microscopy and Spectroscopy in Food and Plant Sciences (VLAG/EPS, NL) 2017

Healthy and Sustainable Diets: Synergies and Trade-offs (VLAG, NL) 2017

\section{Conferences}

The International Union of Food Science and Technology IUFoST

(Dublin, ROI) ${ }^{1}$

10th NIZO Dairy Conference (Ede, NL) ${ }^{2}$

$3^{\text {rd }}$ Food Structure and Functionality Forum Symposium

(Montreal, CaE) ${ }^{1,2}$

\section{General courses}

VLAG PhD Week in Baarlo (VLAG, NL) 2015

Data Management Planning (WGS, NL) 2016

Scientific Writing (WGS, NL) 2016

The Essentials of Scientific Writing and Presenting (WGS, NL) 2016

Philosophy and Ethics of Food Science and Technology (WGS, NL) 2018

\section{Optional activities}

Research proposal 
PhD study trip to Switzerland \& Germany (FPE, WUR, CH/DE) $)^{1,2}$

PhD study trip to Canada (FPE, WUR, CA) $)^{1,2}$

Weekly group meetings (FPE, WUR, NL)

2015-2019

${ }^{1}$ Oral presentation

2 Poster presentation 

The research described in this thesis was financially supported by the China Scholarship Council (grant number 201406820015).

Cover design by Lu Zhang

Printed by ProefschriftMaken 